\title{
Iridium(III) Complexes with Fluorinated Phenyl-tetrazoles as Cyclometalating Ligands: Enhanced Excited-State Energy and Blue Emission ${ }^{*}$
}

\author{
Andrea Baschieri ${ }^{*, a}$ Letizia Sambri, ${ }^{\mathrm{b}}$ Andrea Mazzanti, ${ }^{\mathrm{b}}$ Armando Carlone, ${ }^{\mathrm{a}}$ Filippo Monti ${ }^{*, \mathrm{c}}$ \\ and Nicola Armarolic
}

a Dipartimento di Scienze Fisiche e Chimiche, Università degli Studi dell’Aquila, Via Vetoio, 67100 L'Aquila, Italy
b Dipartimento di Chimica Industriale “Toso Montanari”, Università di Bologna, Viale Risorgimento 4, 40136 Bologna, Italy
${ }^{c}$ Istituto per la Sintesi Organica e la Fotoreattività, Consiglio Nazionale delle Ricerche, Via P. Gobetti 101, 40129 Bologna, Italy

₹ Dedicated to the memory of Prof. Giuseppe Bartoli 


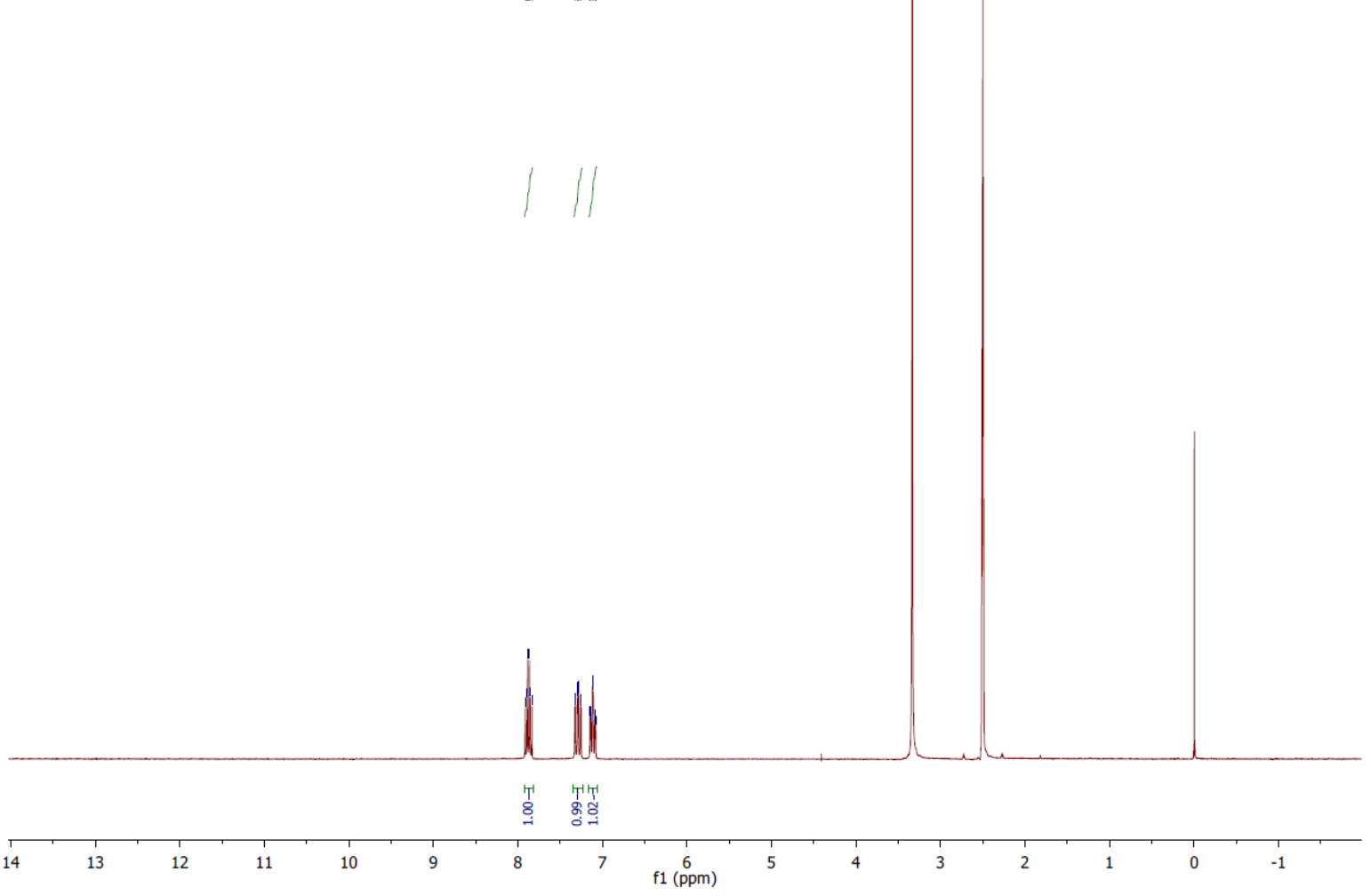

Figure S1a. ${ }^{1} \mathrm{H}$ NMR spectrum of compound II.
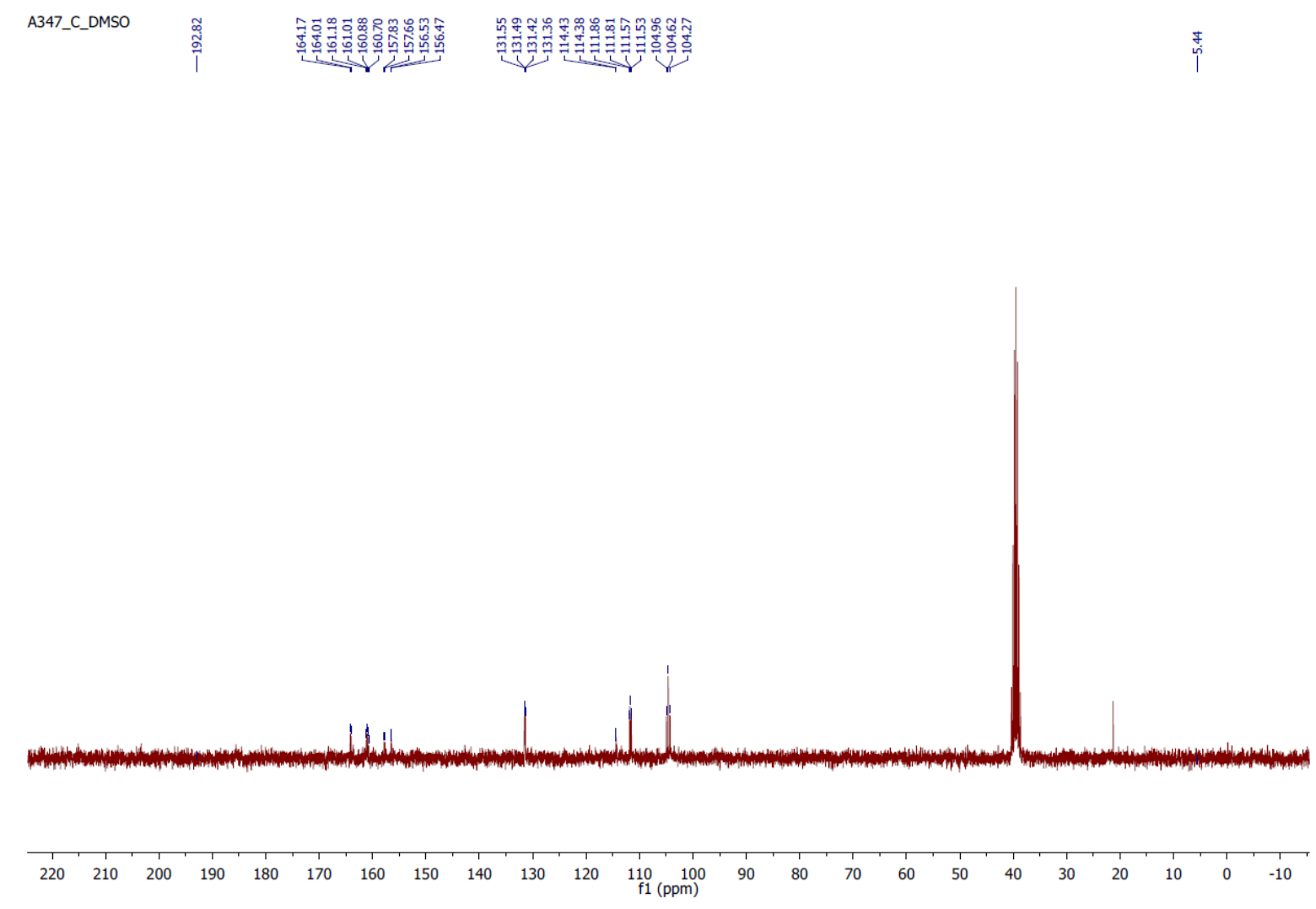

Figure S1b. ${ }^{13} \mathrm{C}$ NMR spectrum of compound II. 


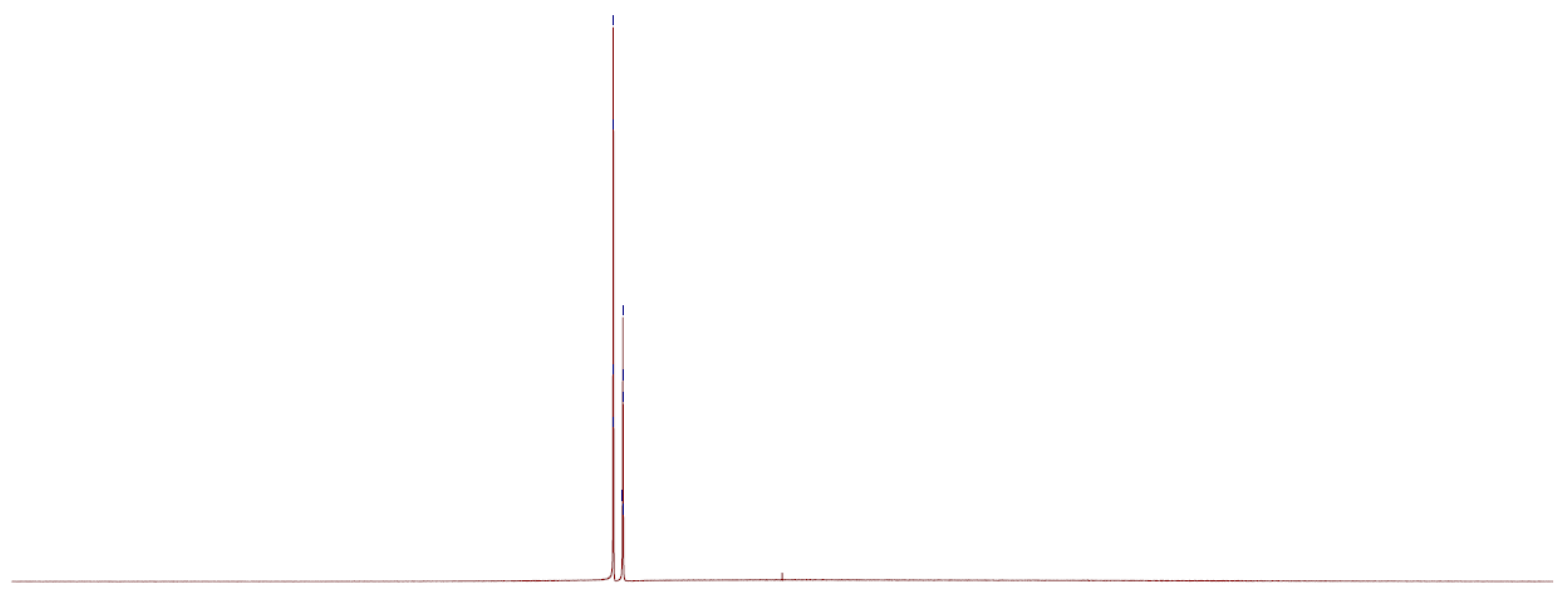

\begin{tabular}{|c|c|c|c|c|c|c|c|c|c|c|c|c|c|c|c|c|c|c|}
\hline & -50 & -60 & $\begin{array}{l}1 \\
-70\end{array}$ & & $\begin{array}{l}1 \\
-90\end{array}$ & $\begin{array}{l}1 \\
-100\end{array}$ & $\begin{array}{c}1 \\
-110\end{array}$ & -120 & $\begin{array}{c}-130 \\
\mathrm{f} 1(\mathrm{ppm})\end{array}$ & -140 & -150 & -160 & -170 & -180 & -190 & -200 & -210 & -220 \\
\hline
\end{tabular}

Figure S1c. ${ }^{19}$ F NMR spectrum of compound II.

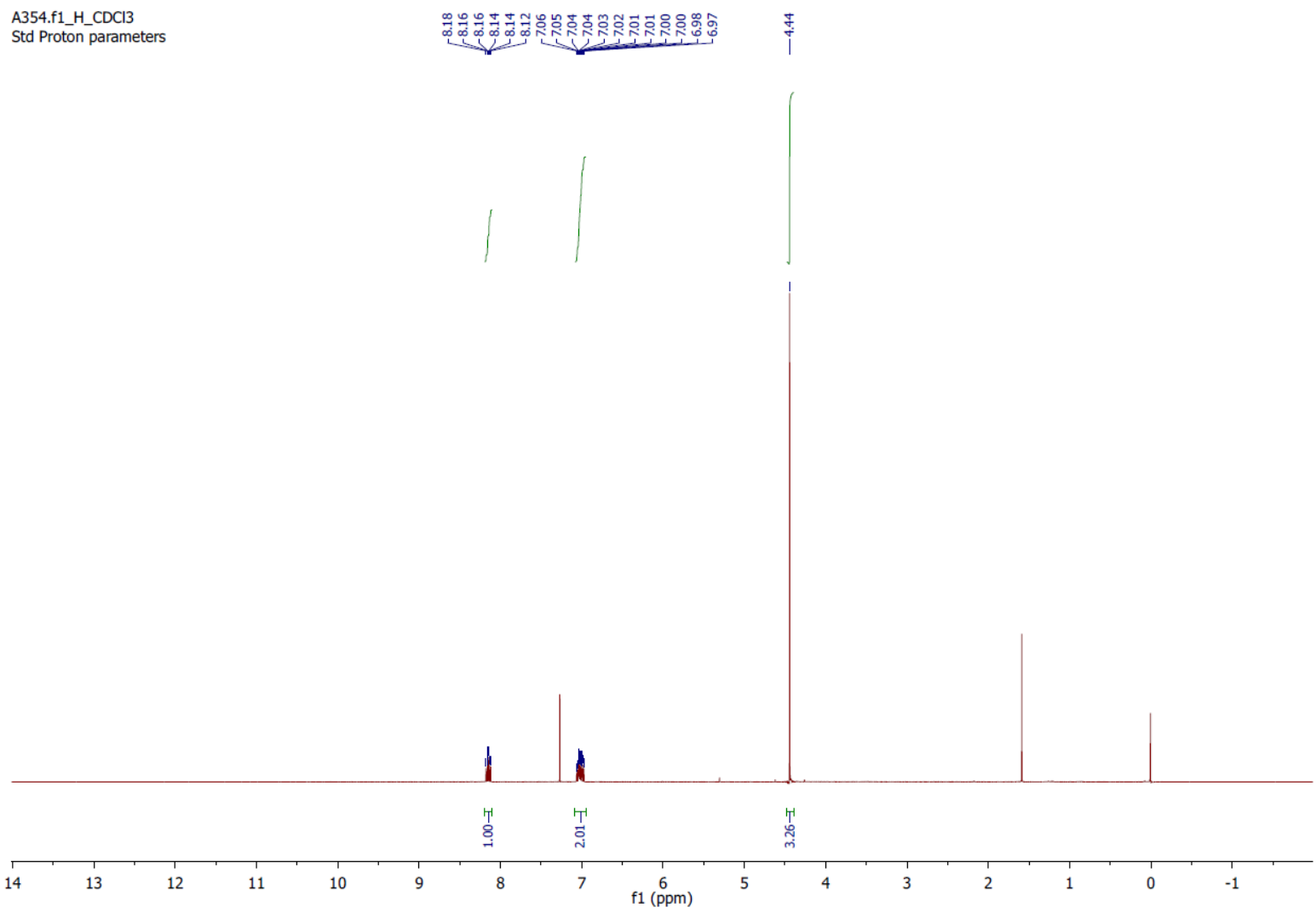

Figure S2a. ${ }^{1} \mathrm{H}$ NMR spectrum of ligand III. 


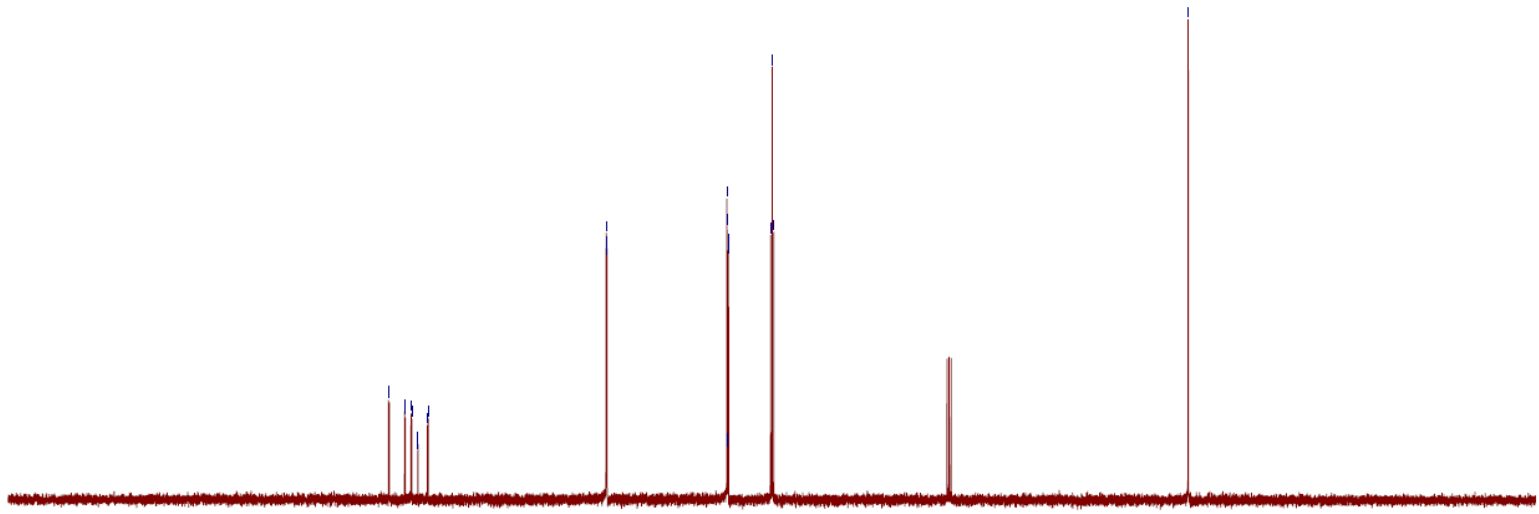

$\begin{array}{llllllllllll}220 & 210 & 200 & 190 & 180 & 170 & 160 & 150 & 140 & 130 & 120 & \begin{array}{l}110 \\ \mathrm{f} 1(\mathrm{ppm})\end{array}\end{array}$

Figure S2b. ${ }^{13} \mathrm{C}$ NMR spectrum of ligand III.

A354.conc.f1_DEPT_CDC13

Heteronuclear polarization transfer experiment

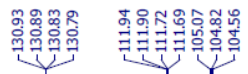

官

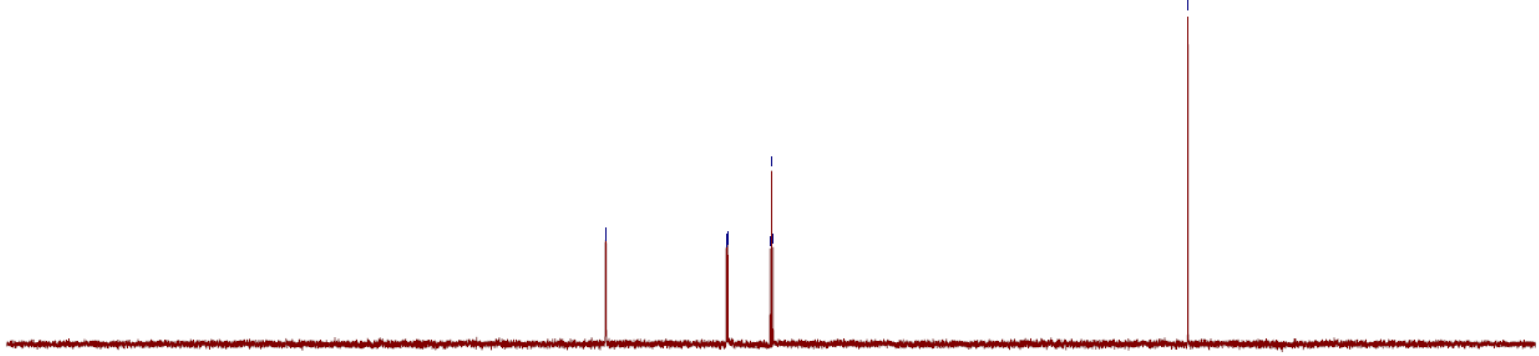

$\begin{array}{llllllllllll}220 & 210 & 200 & 190 & 180 & 170 & 160 & 150 & 140 & 130 & 120 & 110 \\ \mathrm{f} 1(\mathrm{ppm}) & 100\end{array}$

Figure S2c. DEPT spectrum of ligand III. 


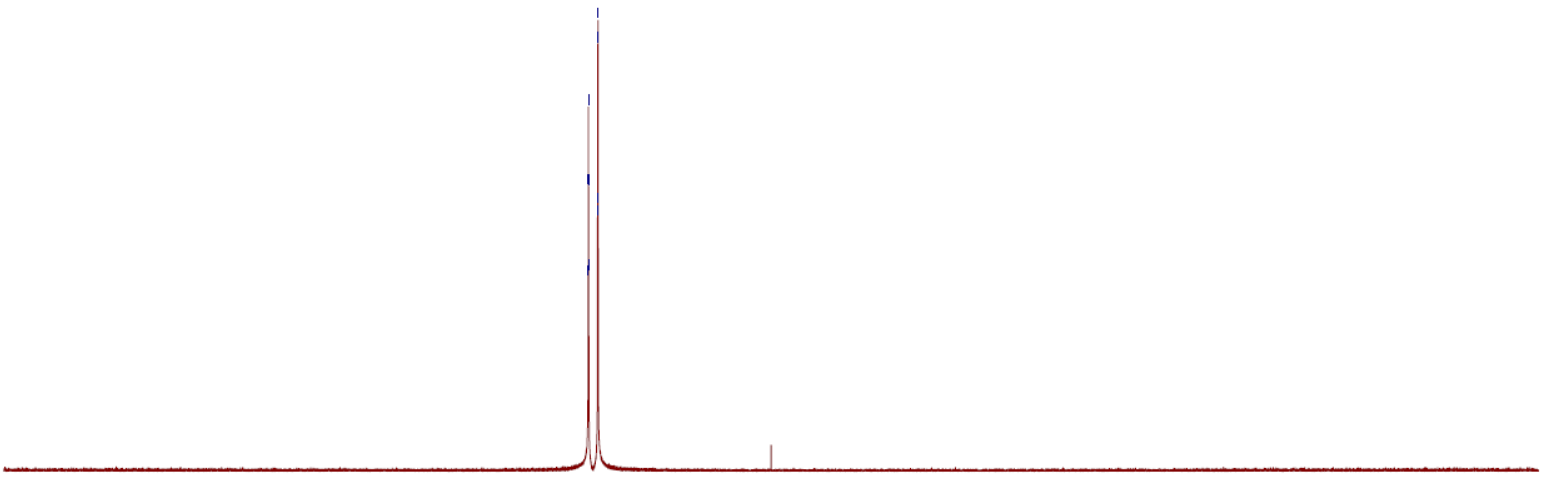

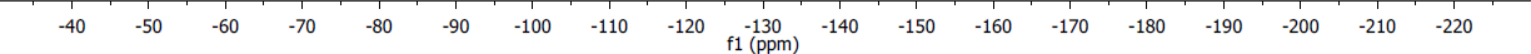

Figure S2d. ${ }^{19} \mathrm{~F}$ NMR spectrum of ligand III.

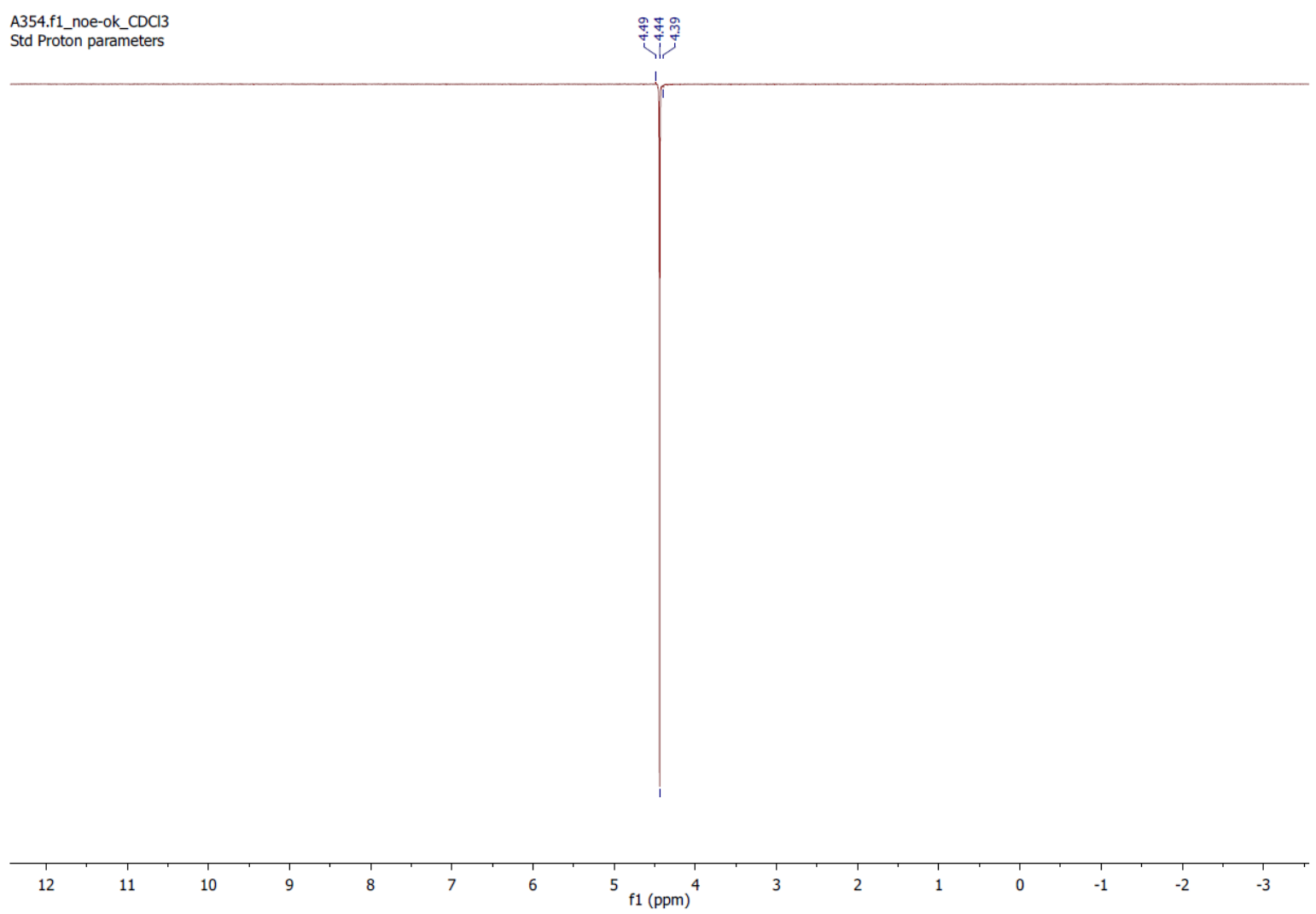

Figure S2e. NOE (4.44 ppm) spectrum of ligand III. 


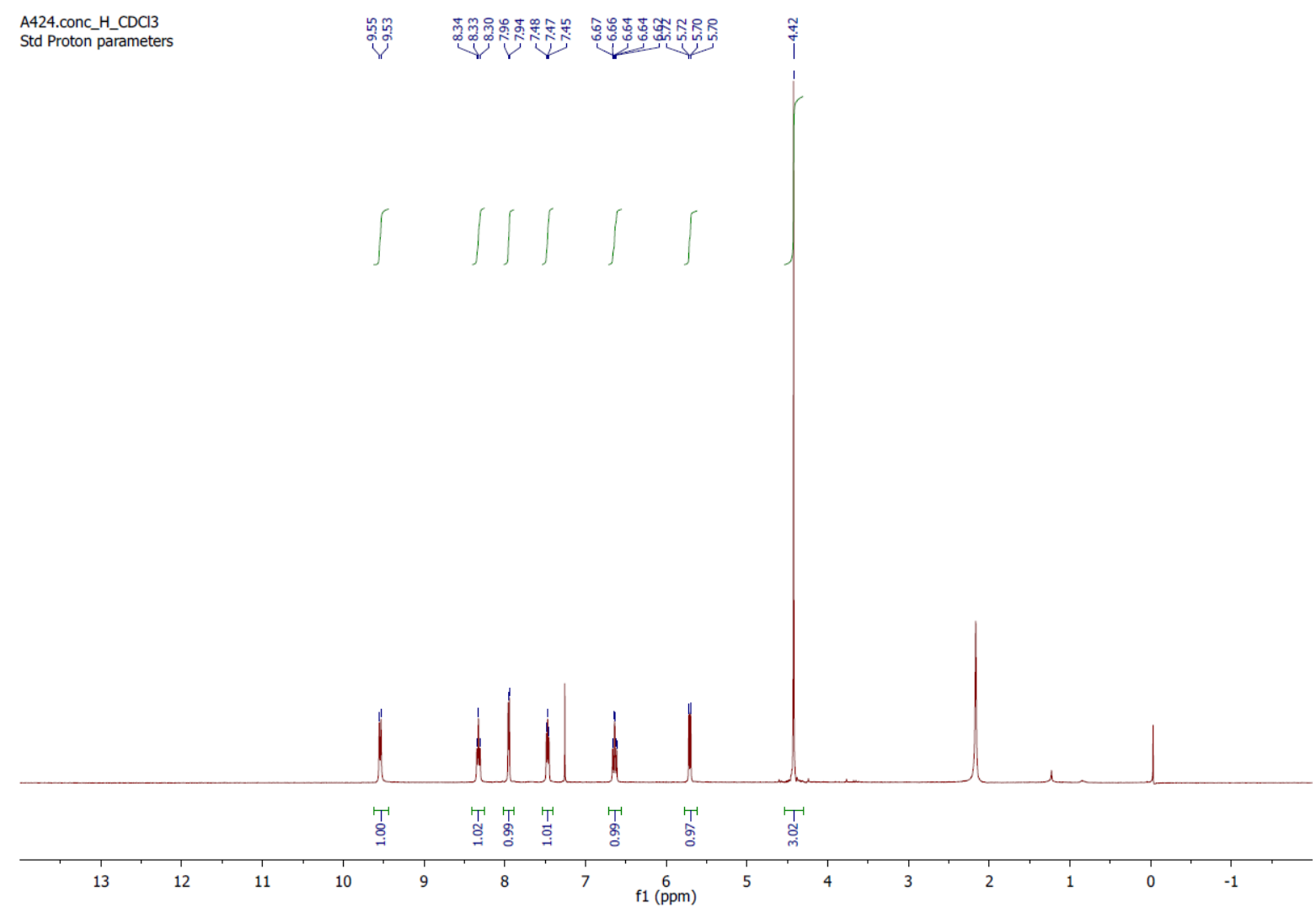

Figure S3a. ${ }^{1} \mathrm{H}$ NMR spectrum of complex $1 \mathrm{~F}$.

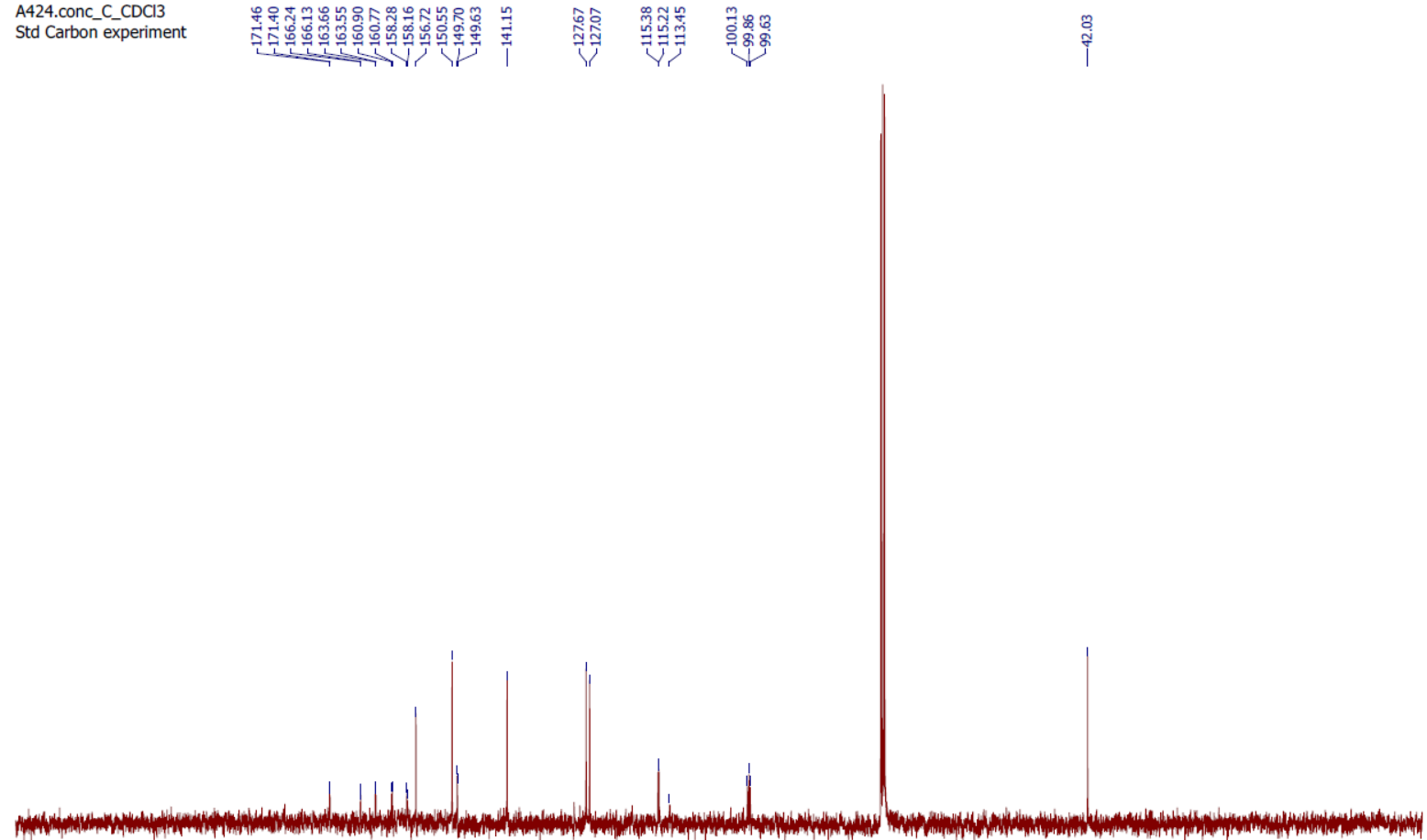

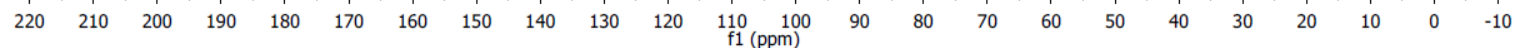

Figure S3b. ${ }^{13} \mathrm{C}$ NMR spectrum of complex $1 F$. 


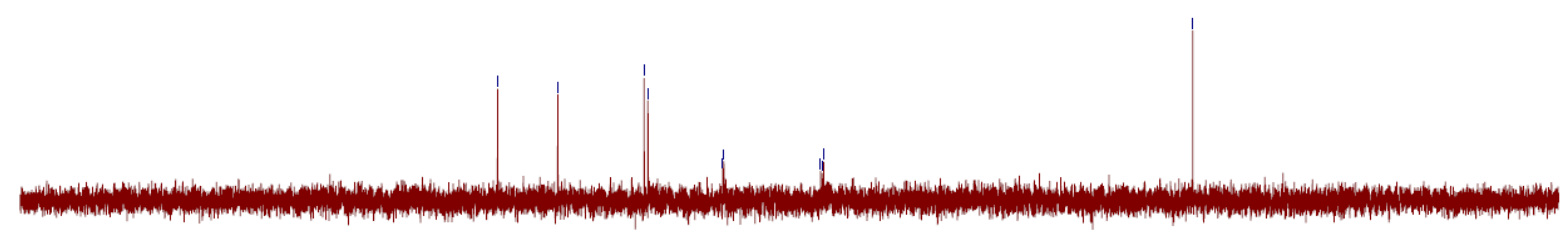

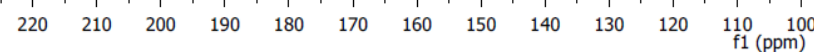

Figure S3c. DEPT NMR spectrum of complex 1F.

A424.scambioPF6_F_CDCl3 Std Fluorine
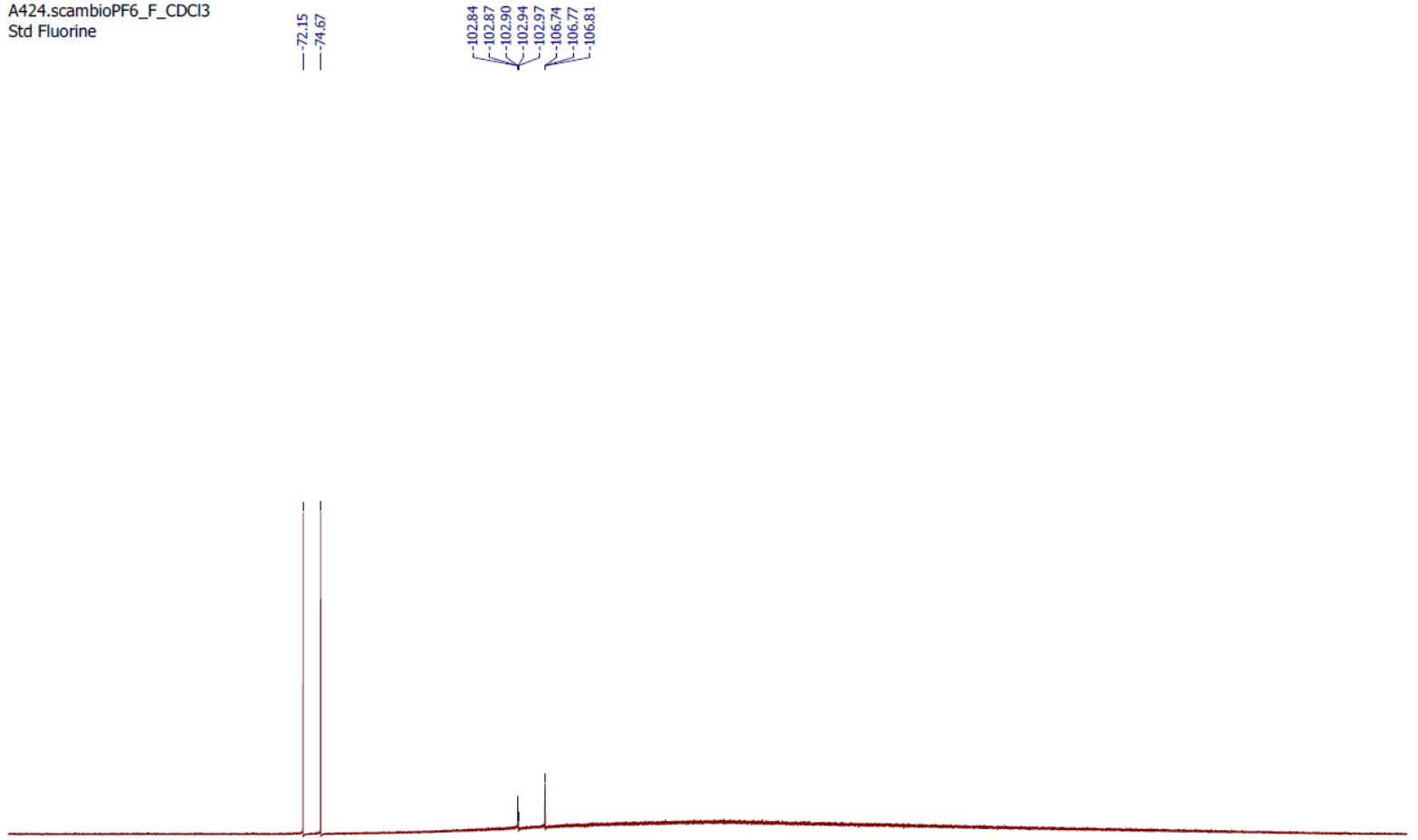

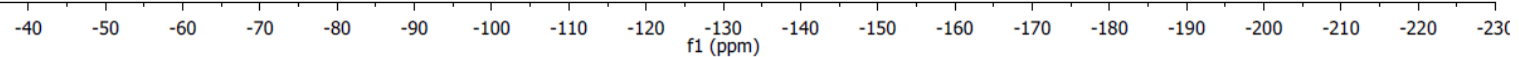

Figure S3d. ${ }^{19}$ F NMR spectrum of complex 1F. 


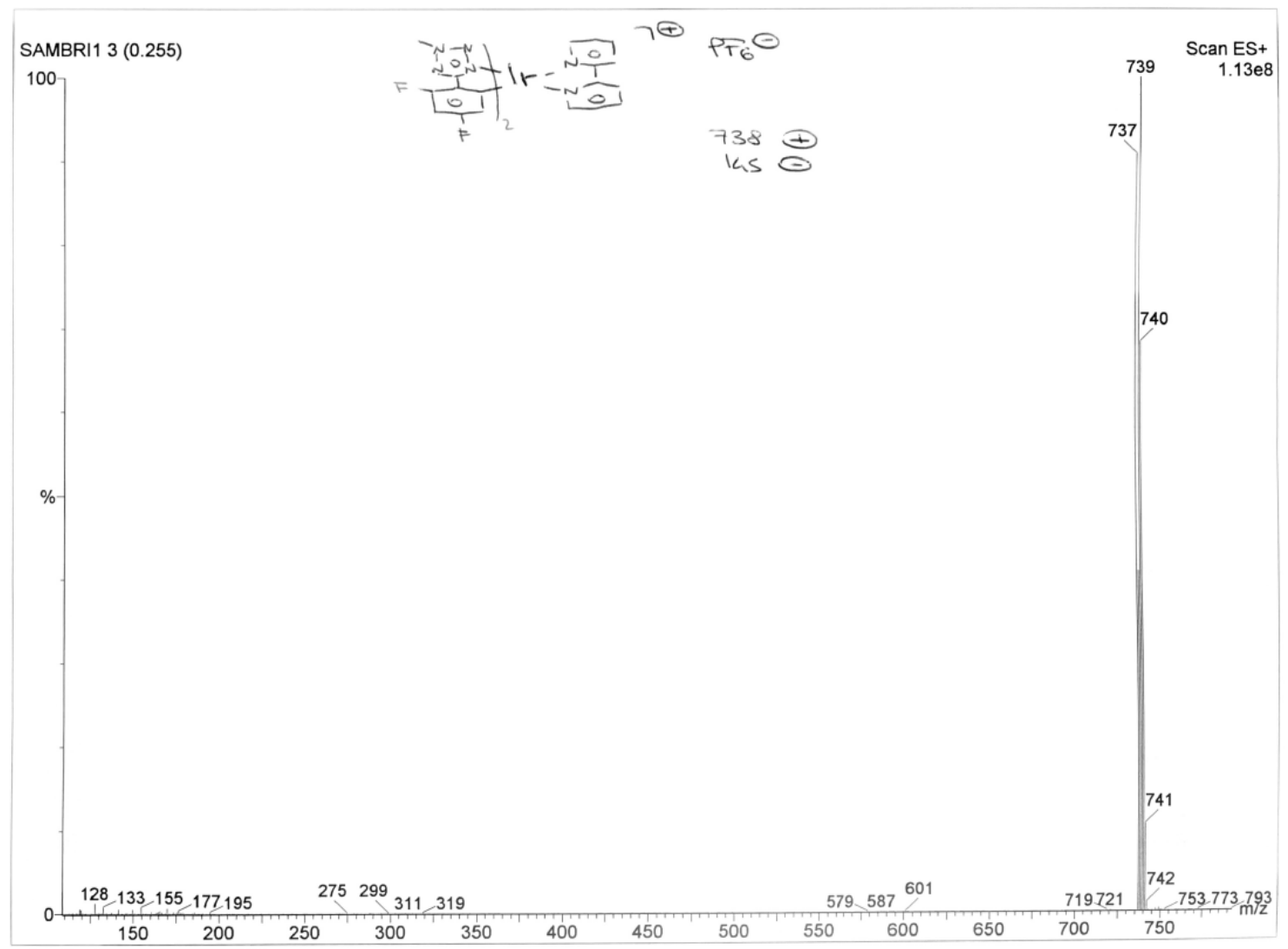

Figure S3e. ESI ${ }^{+}$spectrum of complex 1F.

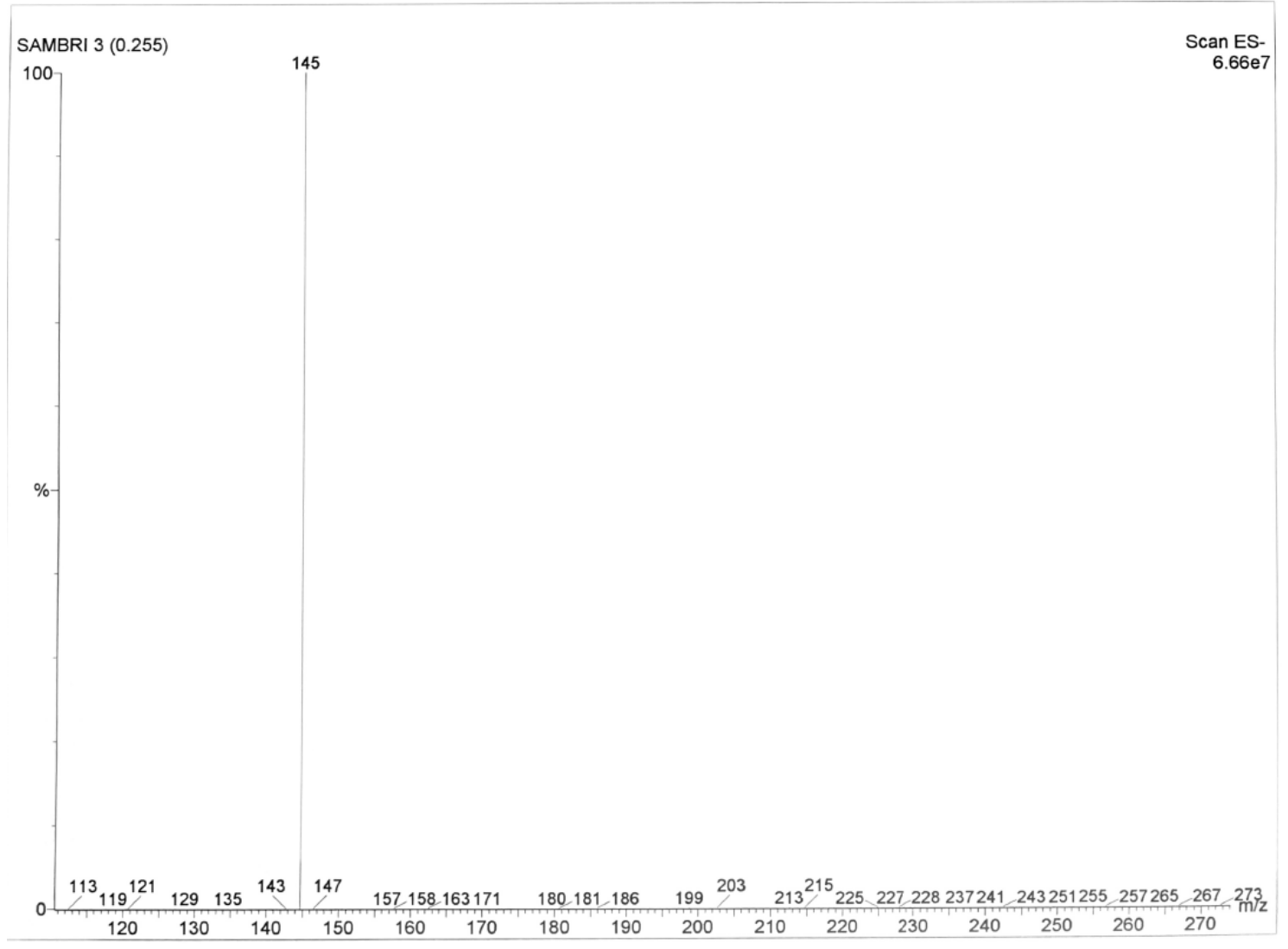

Figure S3f. ESI- ${ }^{-}$spectrum of complex 1F. 


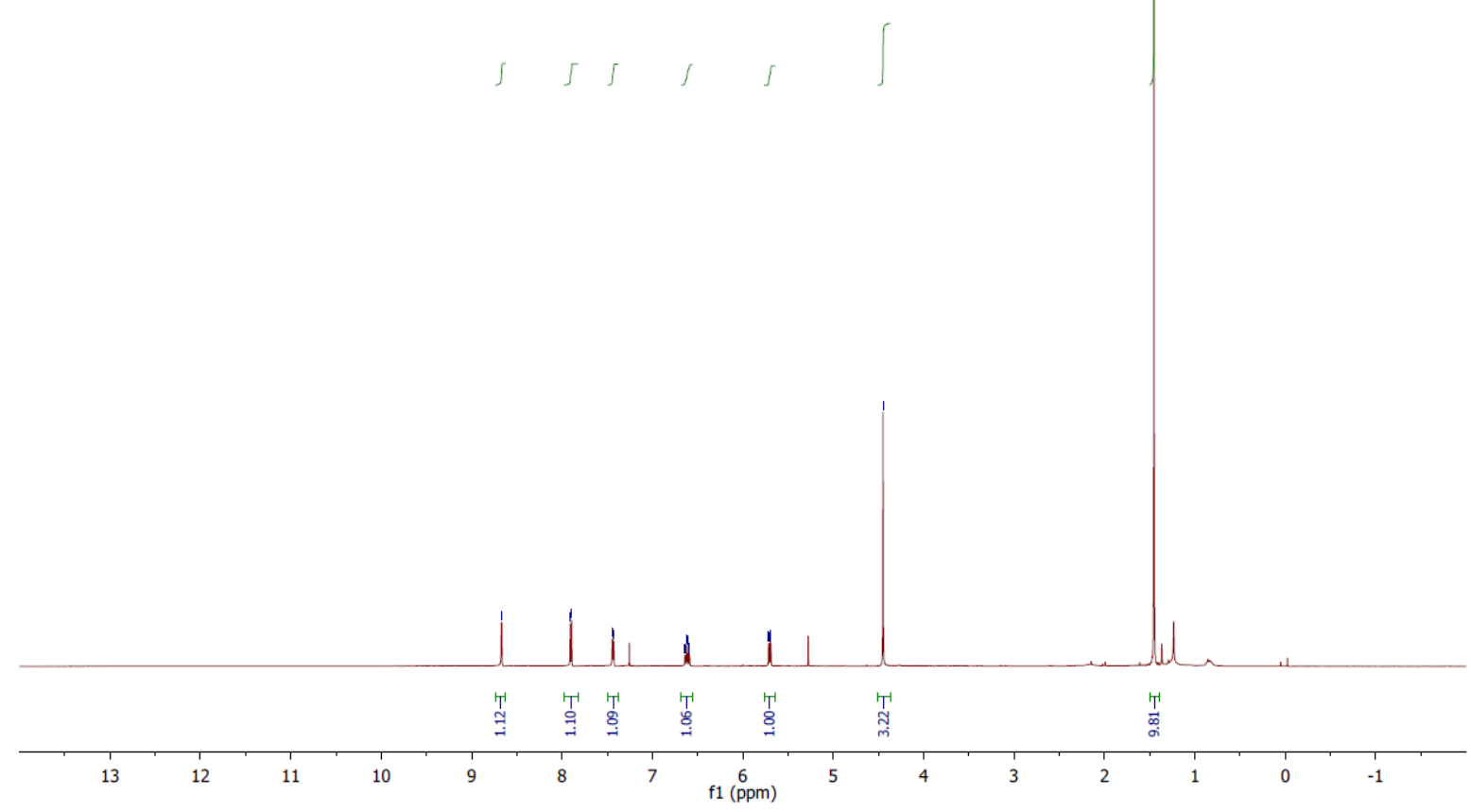

Figure S4a. ${ }^{1} \mathrm{H}$ NMR spectrum of complex 2 F.

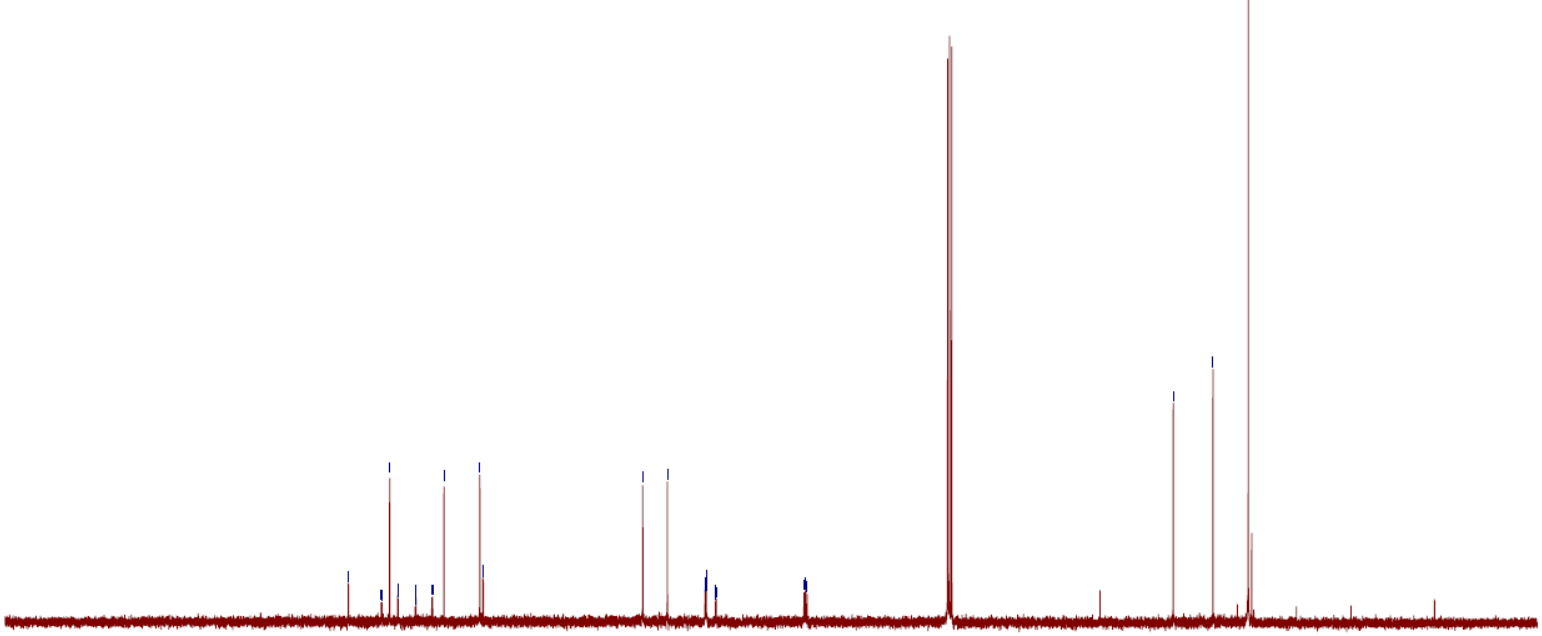

$\begin{array}{llllllllllll}220 & 210 & 200 & 190 & 180 & 170 & 160 & 150 & 140 & 130 & 120 & \begin{array}{c}110 \\ \mathrm{f} 1(\mathrm{ppm})\end{array}\end{array}$

Figure S4b. ${ }^{13} \mathrm{C}$ NMR spectrum of complex 2F. 


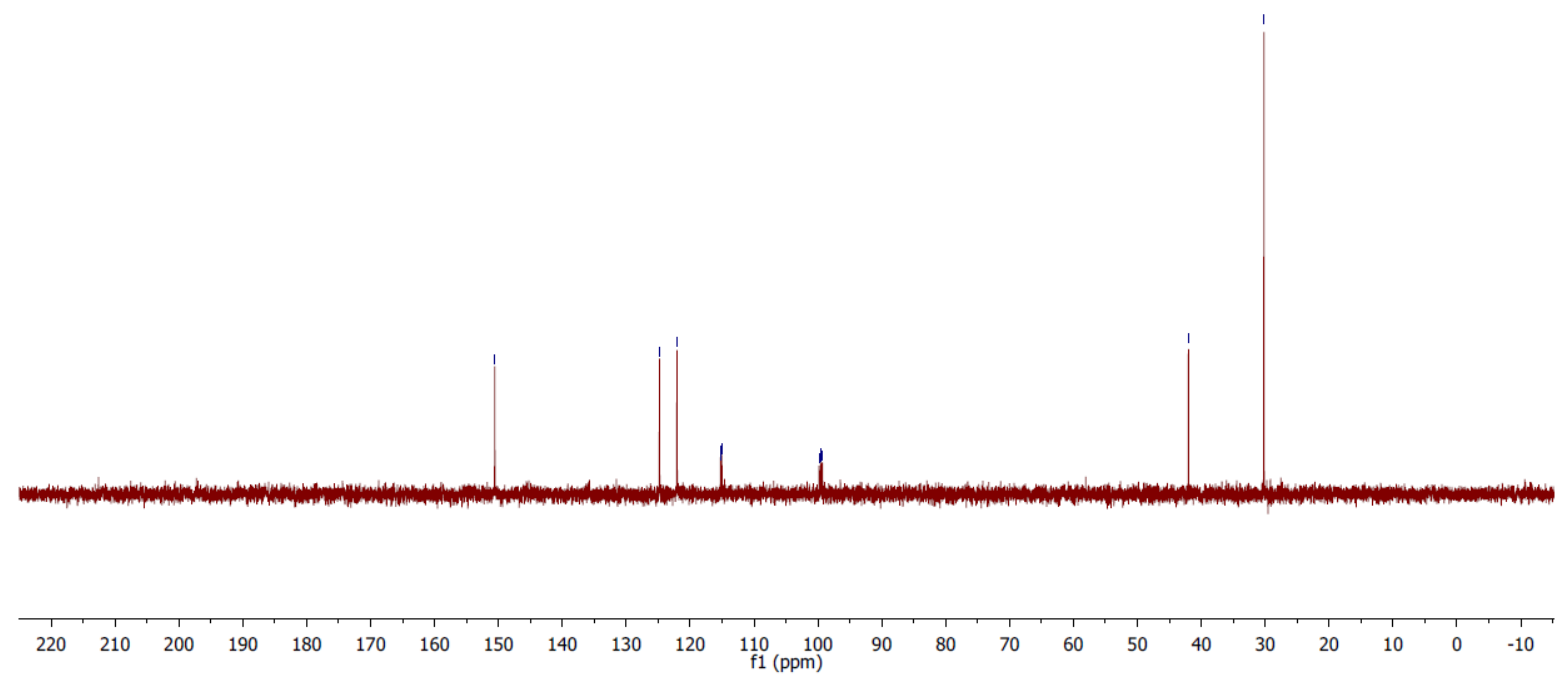

Figure S4c. DEPT NMR spectrum of complex 2F.

A422scambio_F_CDCl3 Std Fluorine

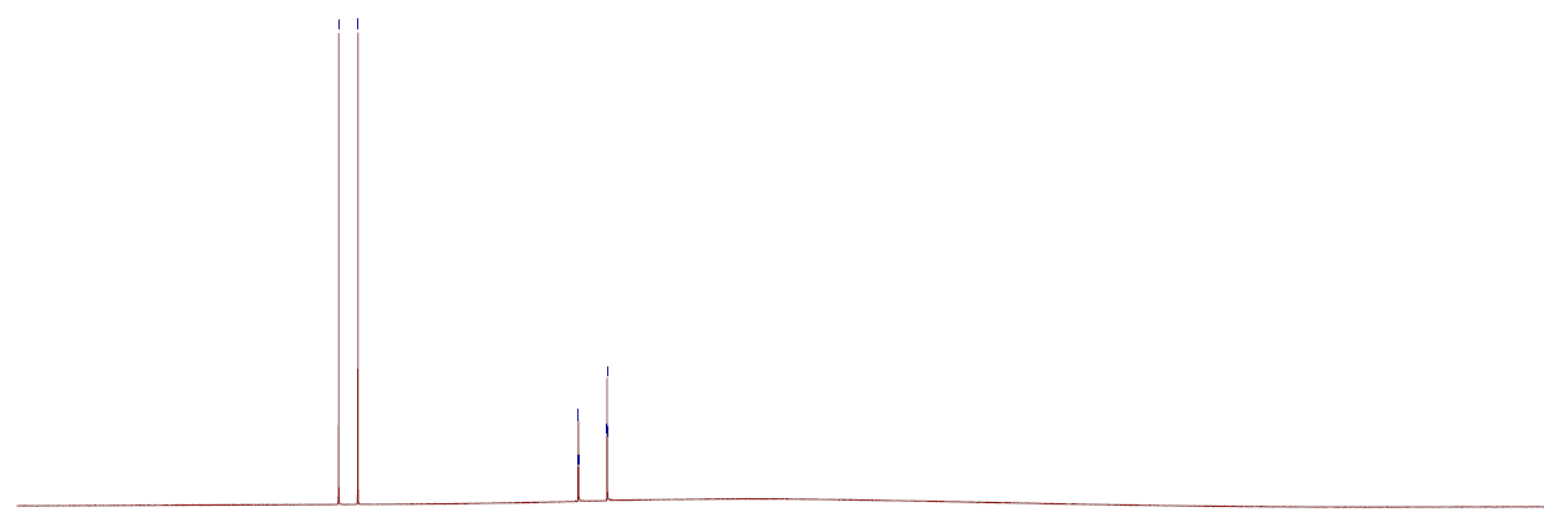

$\begin{array}{llllllll}-40 & -50 & -60 & -70 & -80 & -90 & -100 & -110\end{array}$

$\begin{array}{cc}-120 & -130 \\ \mathrm{f} 1 & (\mathrm{ppm})\end{array}$

$-150$

$\begin{array}{llllll}-180 & -190 & -200 & -210 & -220 & -23\end{array}$

Figure S4d. ${ }^{19} \mathrm{~F}$ NMR spectrum of complex 2F. 


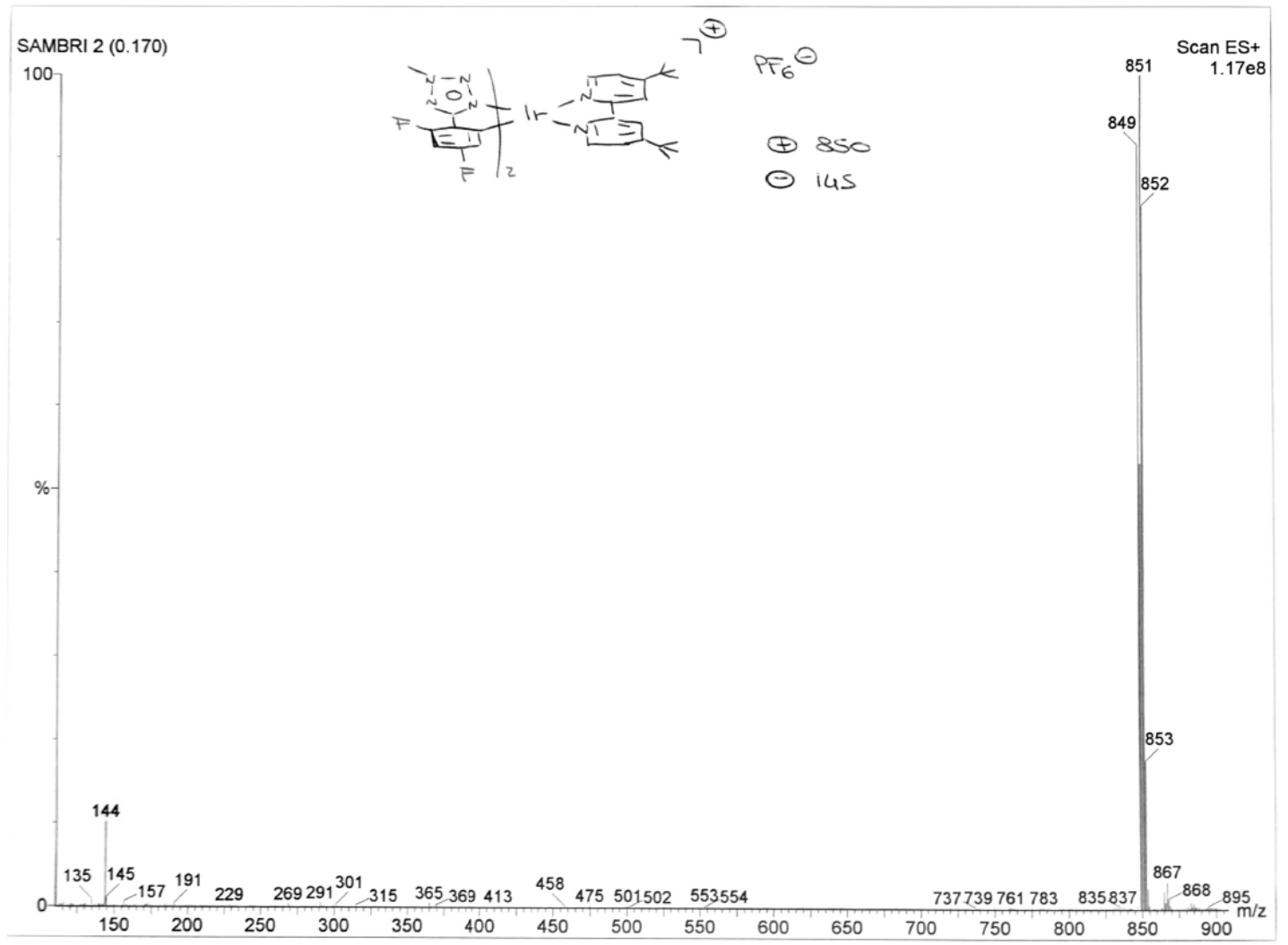

Figure S4e. $\mathrm{ESI}^{+}$spectrum of complex 2F.

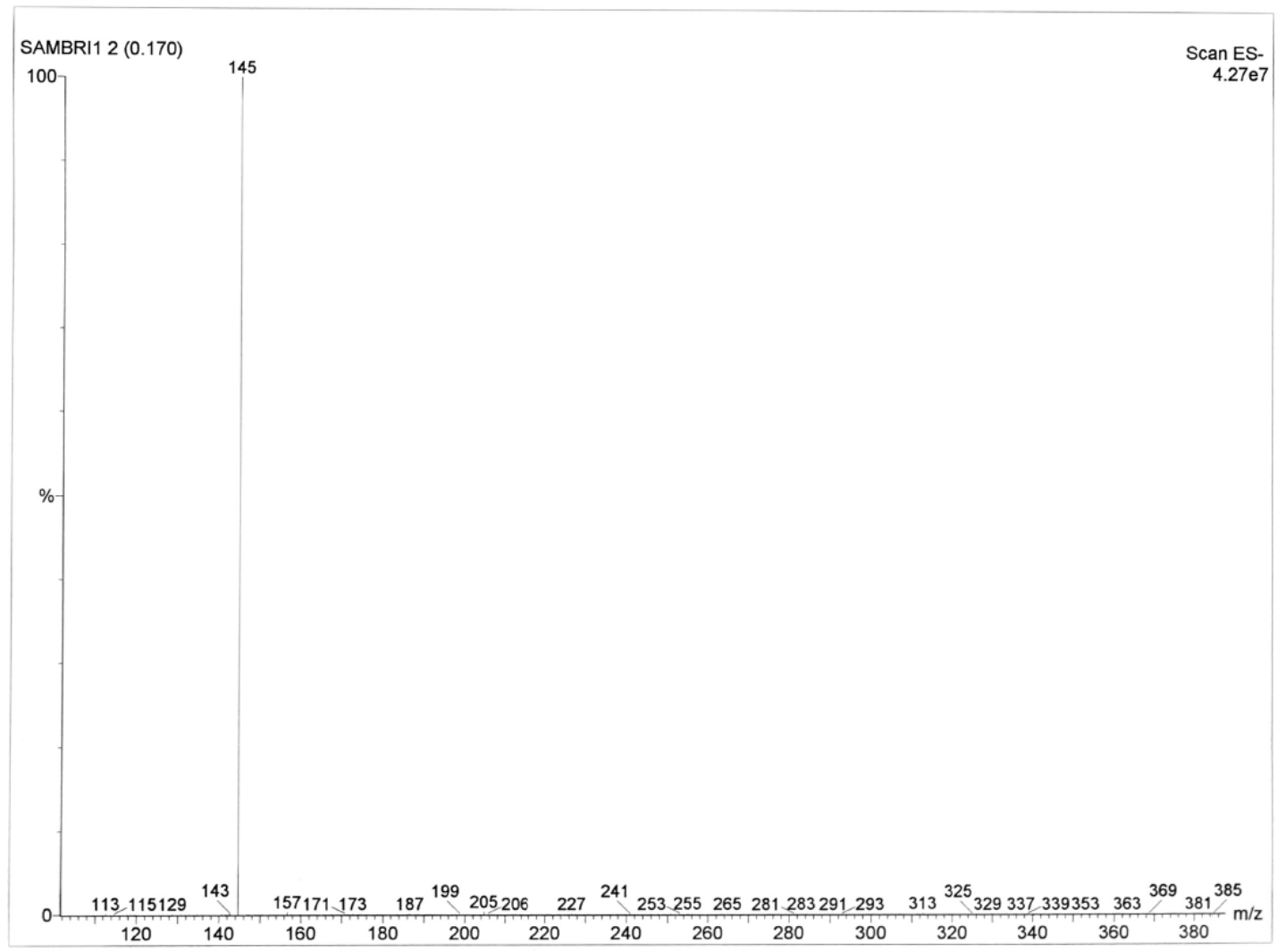

Figure S4f. ESI- ${ }^{-}$spectrum of complex 2F. 


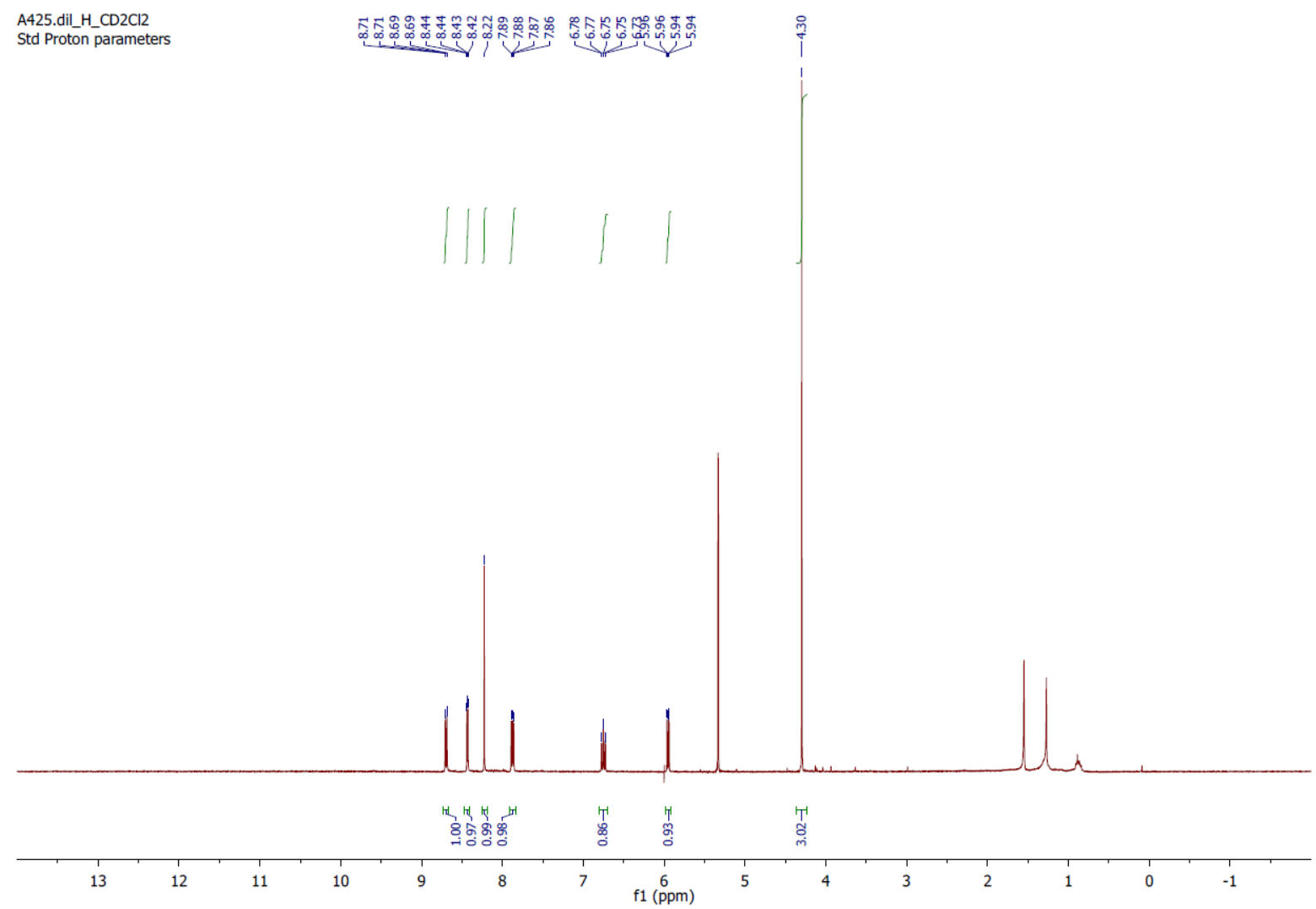

Figure S5a. ${ }^{1} \mathrm{H}$ NMR spectrum of complex 3F.
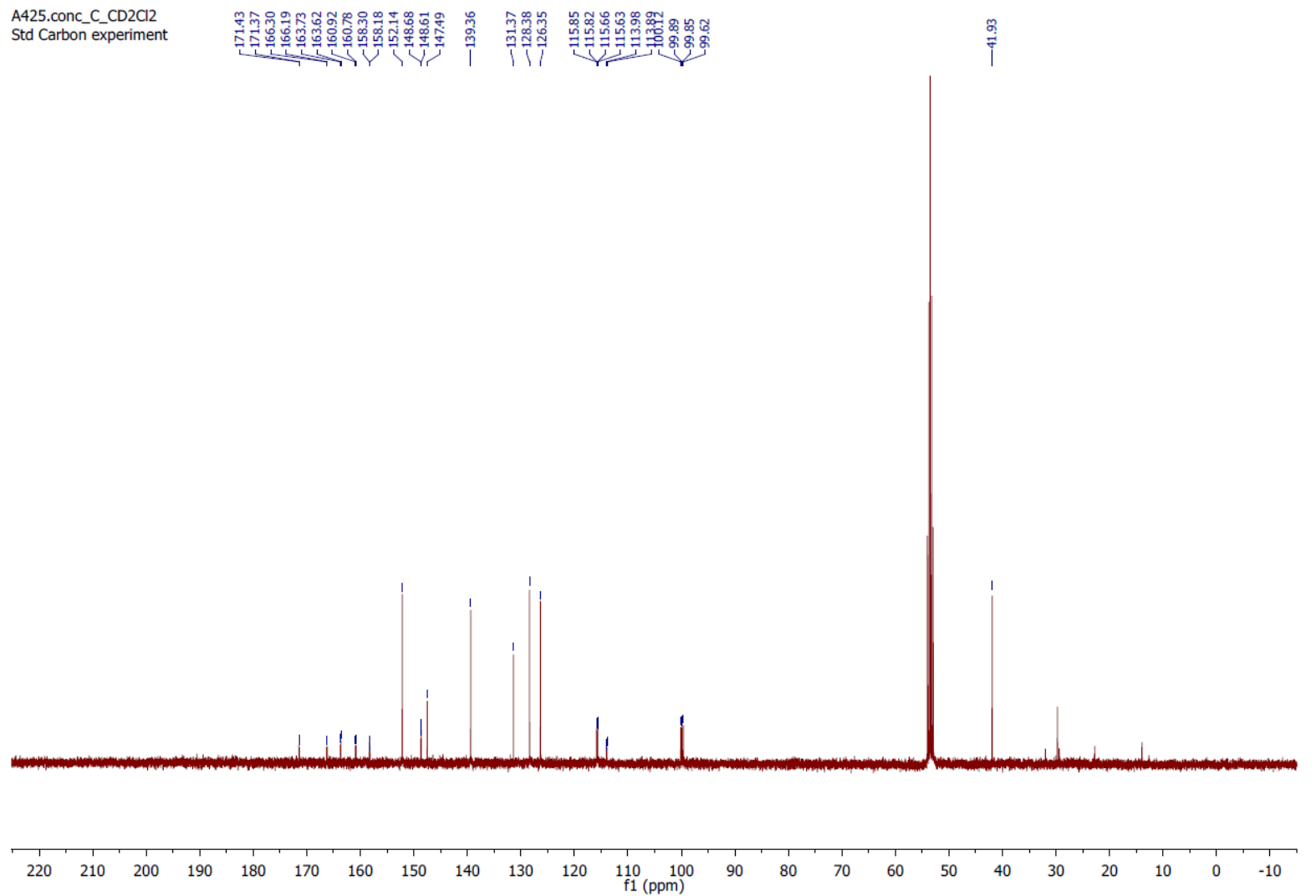

Figure S5b. ${ }^{13} \mathrm{C}$ NMR spectrum of complex 3F. 


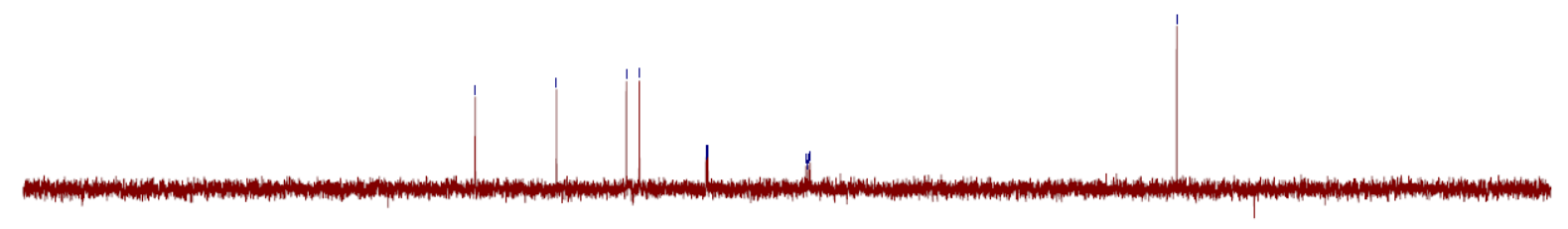

$\begin{array}{lllllllllllllllllllllllllll}220 & 210 & 200 & 190 & 180 & 170 & 160 & 150 & 140 & 130 & 120 & 110 & 100 & 90 & 80 & 70 & 60 & 50 & 40 & 30 & 20 & 10 & 0 & -10\end{array}$

Figure S5c. DEPT NMR spectrum of complex 3F.

A425.dil_F_CD2C12

Std Fluorine experiment
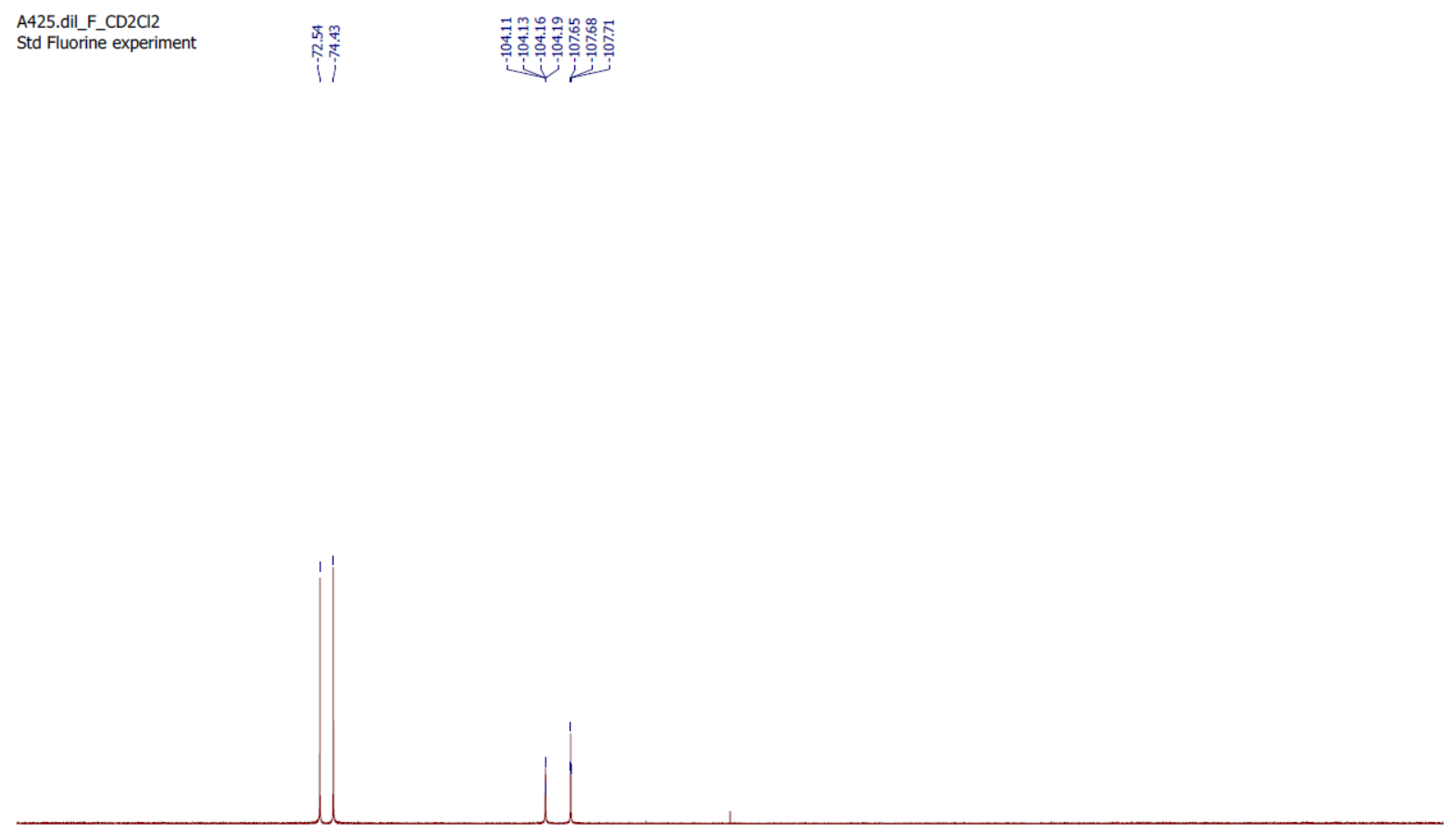

Figure S5d. ${ }^{19}$ F NMR spectrum of complex 3F. 


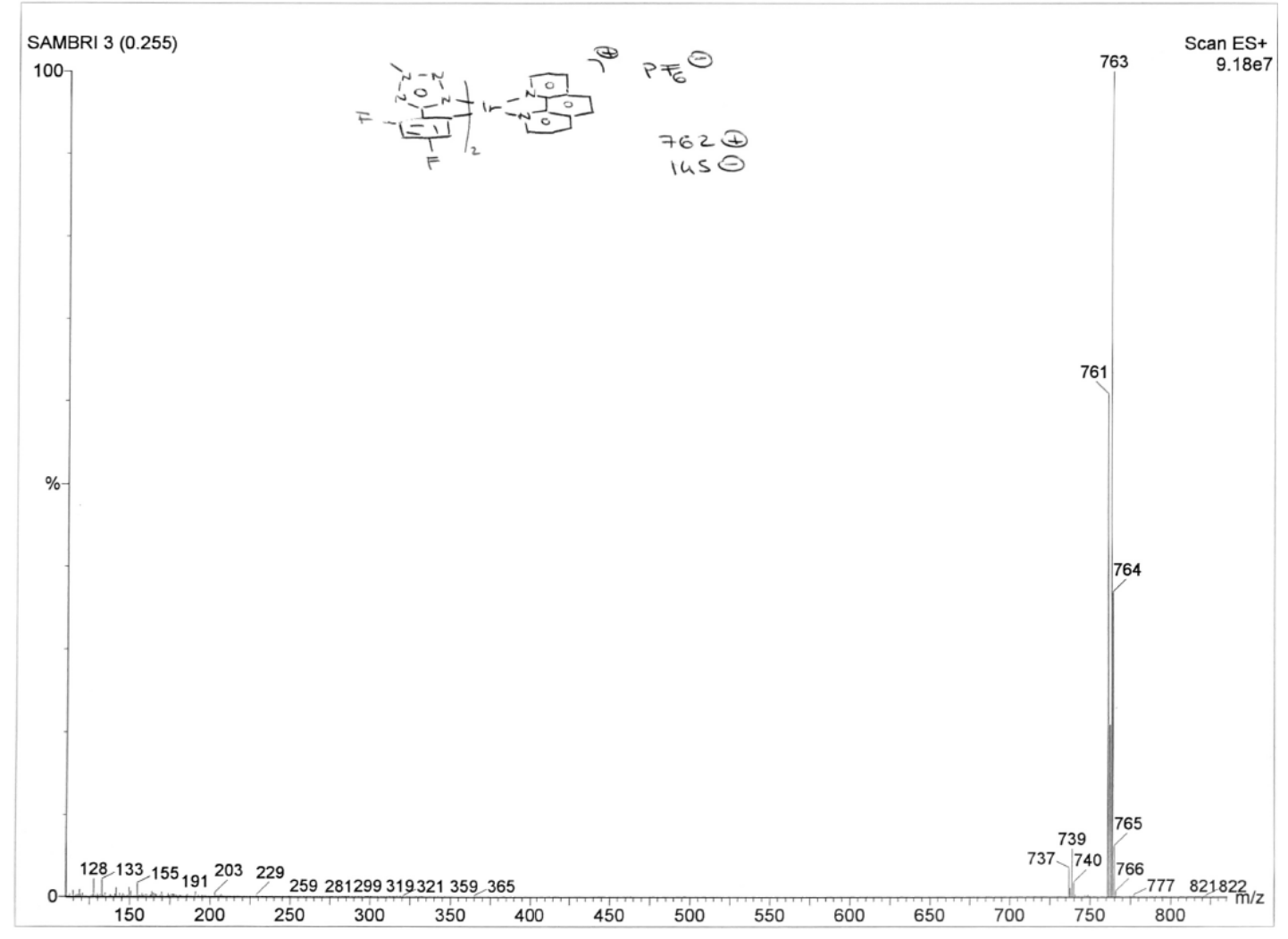

Figure S5e. ESI ${ }^{+}$spectrum of complex 3F.

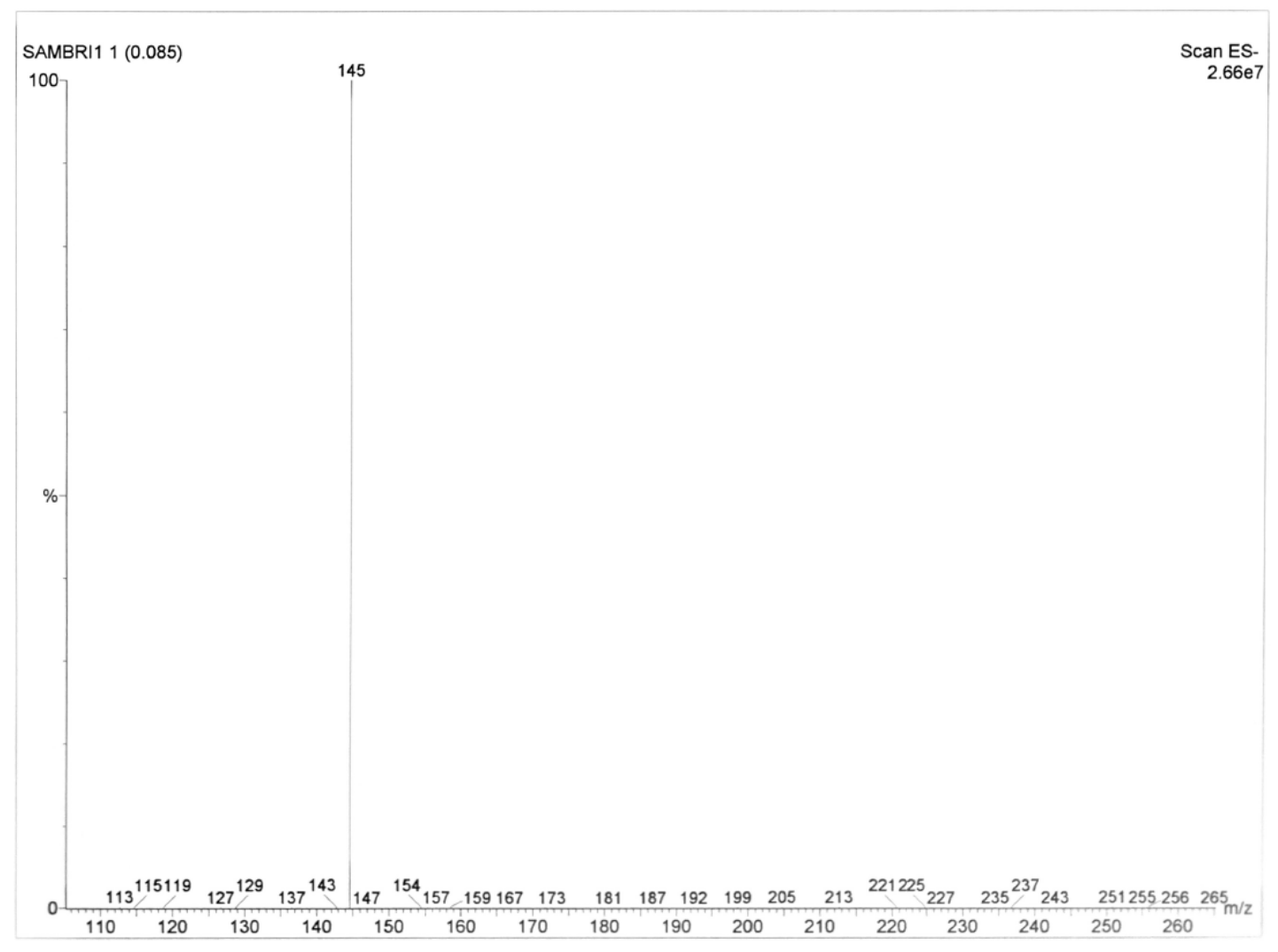

Figure S5f. ESI- spectrum of complex 3F. 


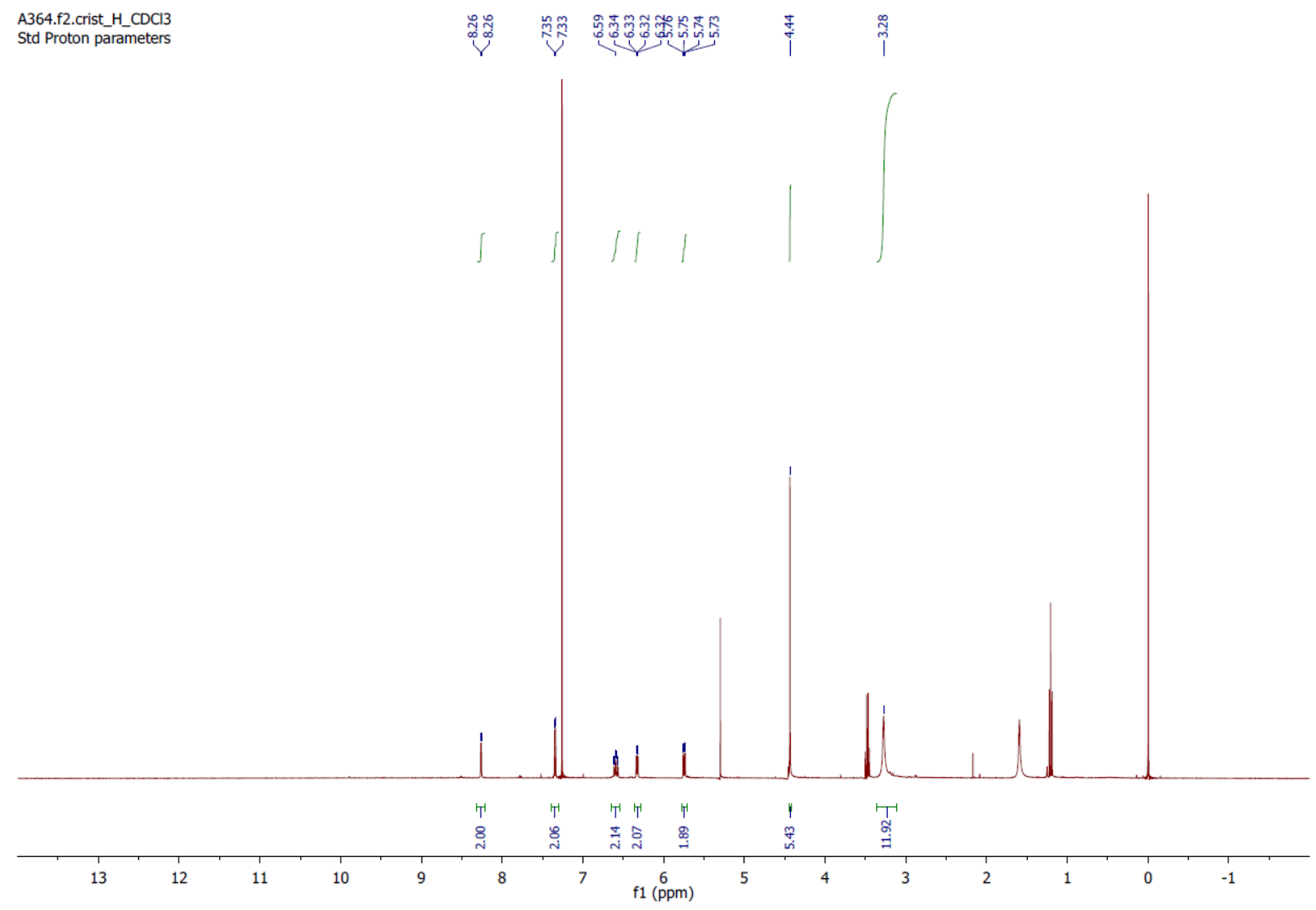

Figure S6a. ${ }^{1} \mathrm{H}$ NMR spectrum of complex 4F.

A364.f2.pur_C_CDCl3

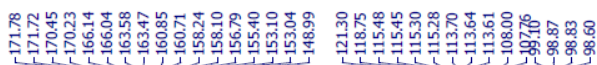

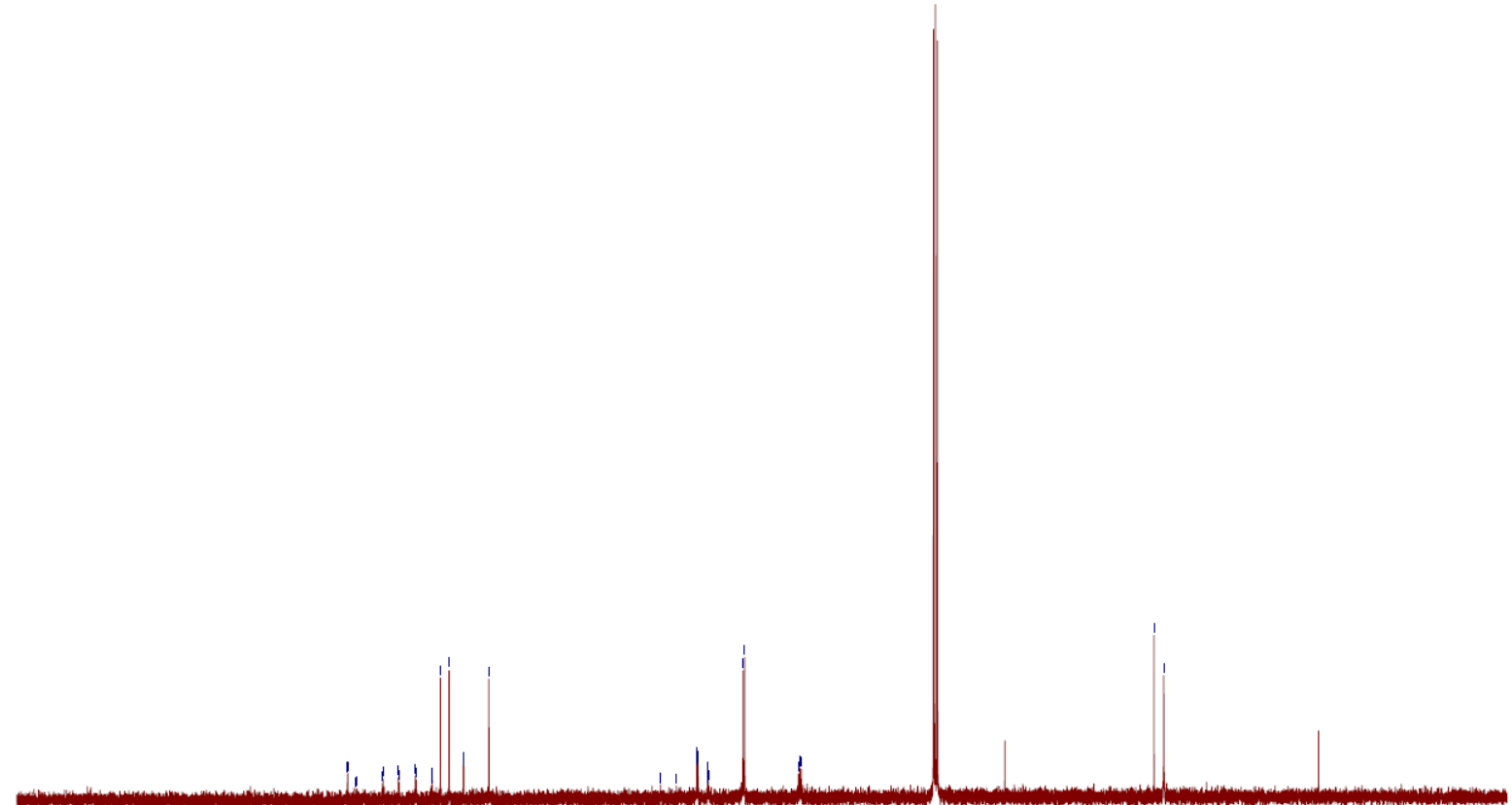

$\begin{array}{llllllllllll}220 & 210 & 200 & 190 & 180 & 170 & 160 & 150 & 140 & 130 & 120 & \underset{f 10}{110}\left(\begin{array}{ll}100 \\ \text { (ppm) }\end{array}\right.\end{array}$

$\begin{array}{lll}90 & 80 & 70\end{array}$ $-10$

Figure S6b. ${ }^{13} \mathrm{C}$ NMR spectrum of complex 4F. 


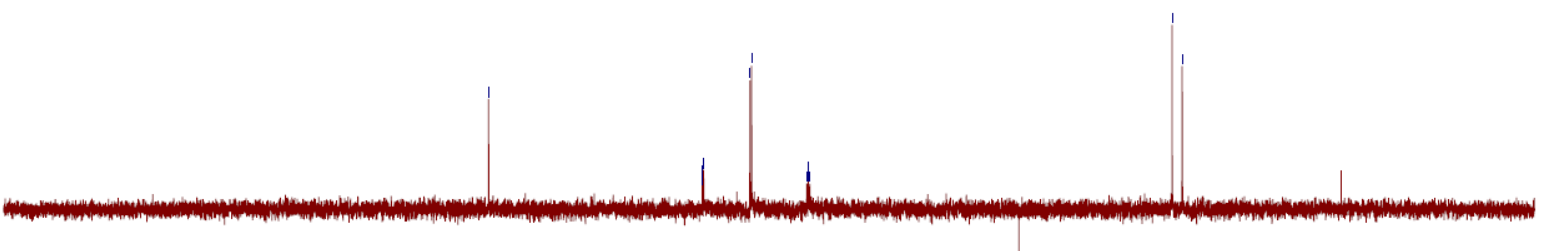

$\begin{array}{llllllllllll}220 & 210 & 200 & 190 & 180 & 170 & 160 & 150 & 140 & 130 & 120 & 110 \\ \mathrm{f} 1(\mathrm{ppm}) & 100\end{array}$

Figure S6c. DEPT NMR spectrum of complex 4F.

A364.f2.crist_F_CDCl3 Std Fluorine experiment
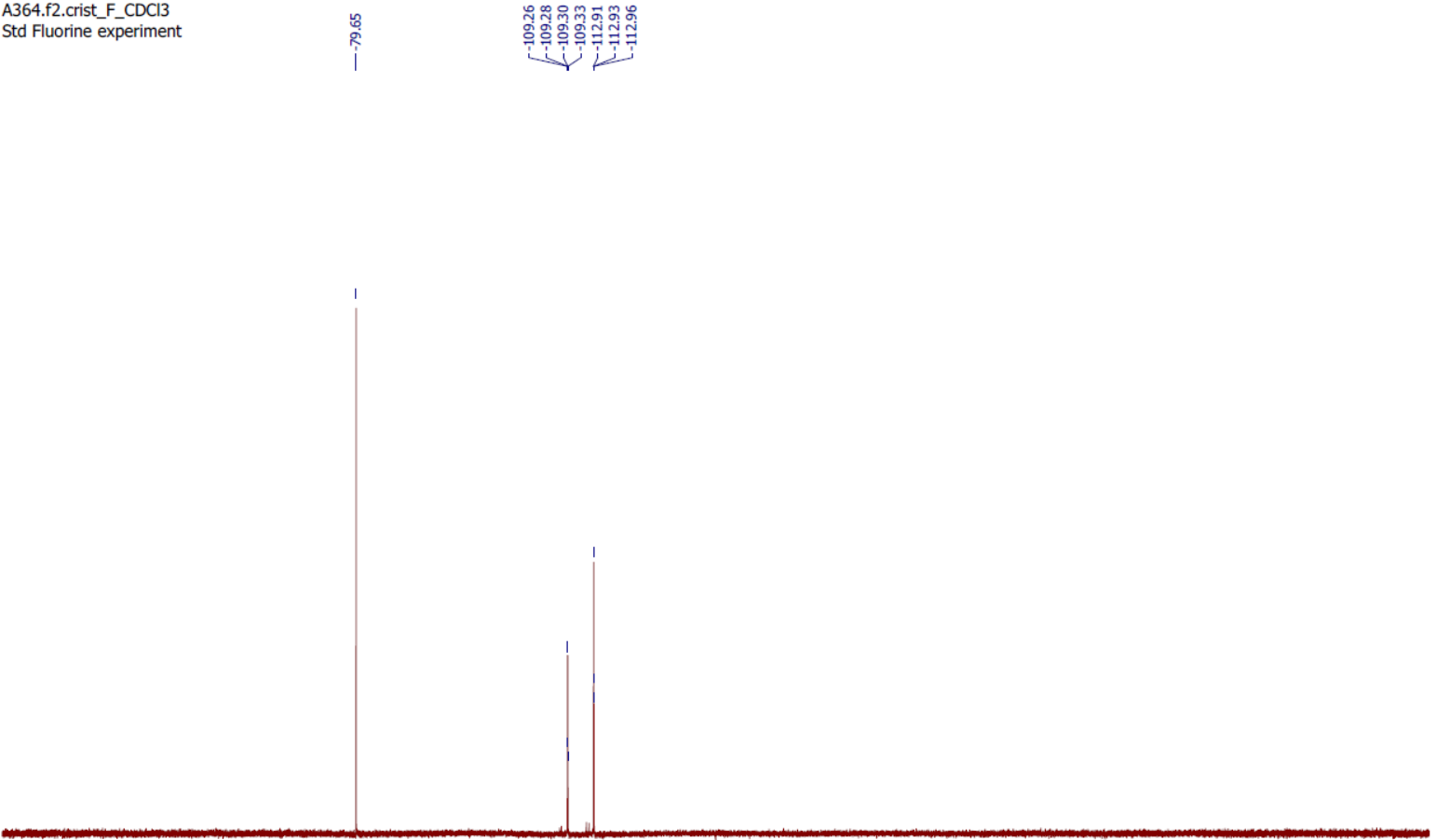

Figure S6d. ${ }^{19} \mathrm{~F}$ NMR spectrum of complex 4F. 


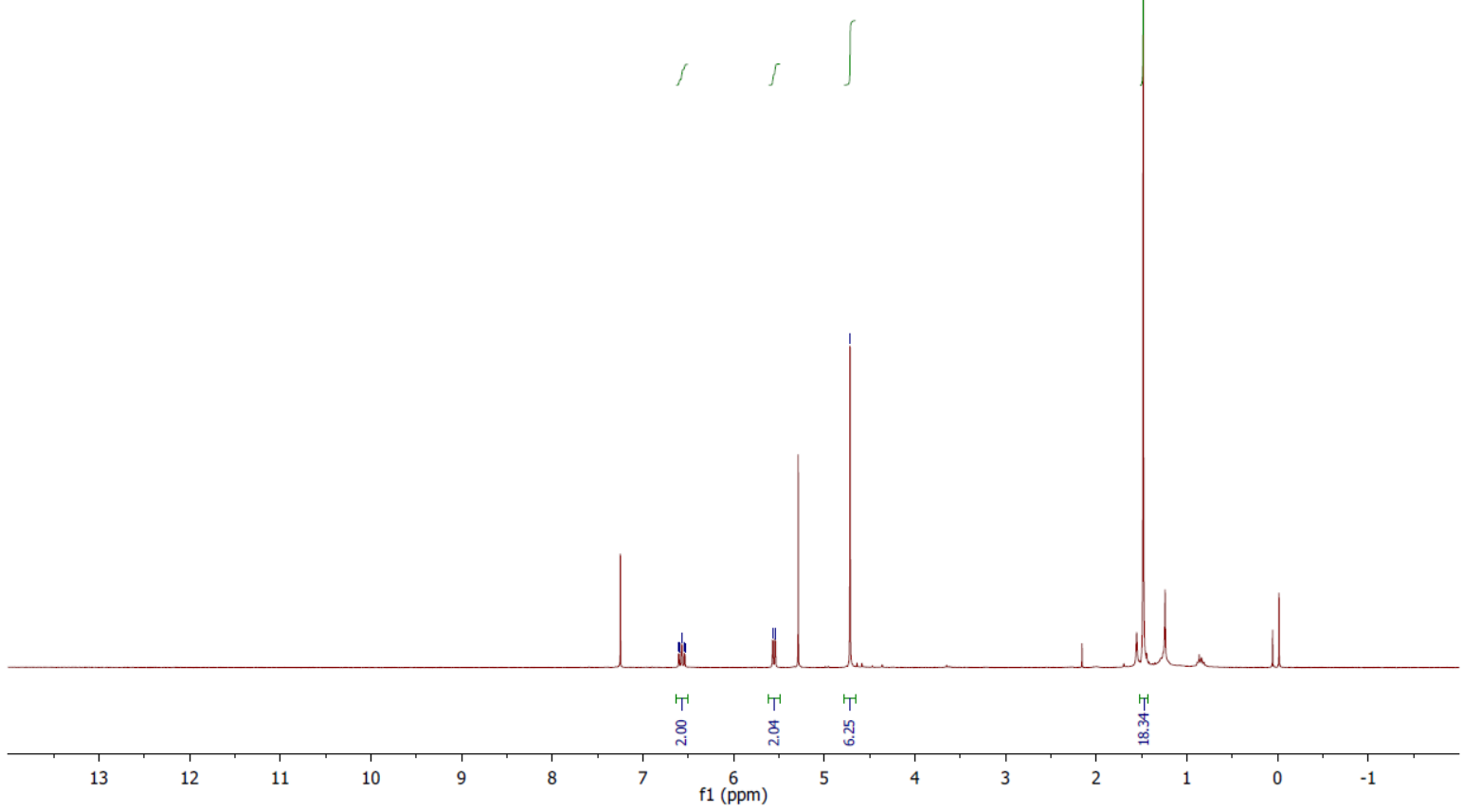

Figure S7a. ${ }^{1} \mathrm{H}$ NMR spectrum of complex 5F.

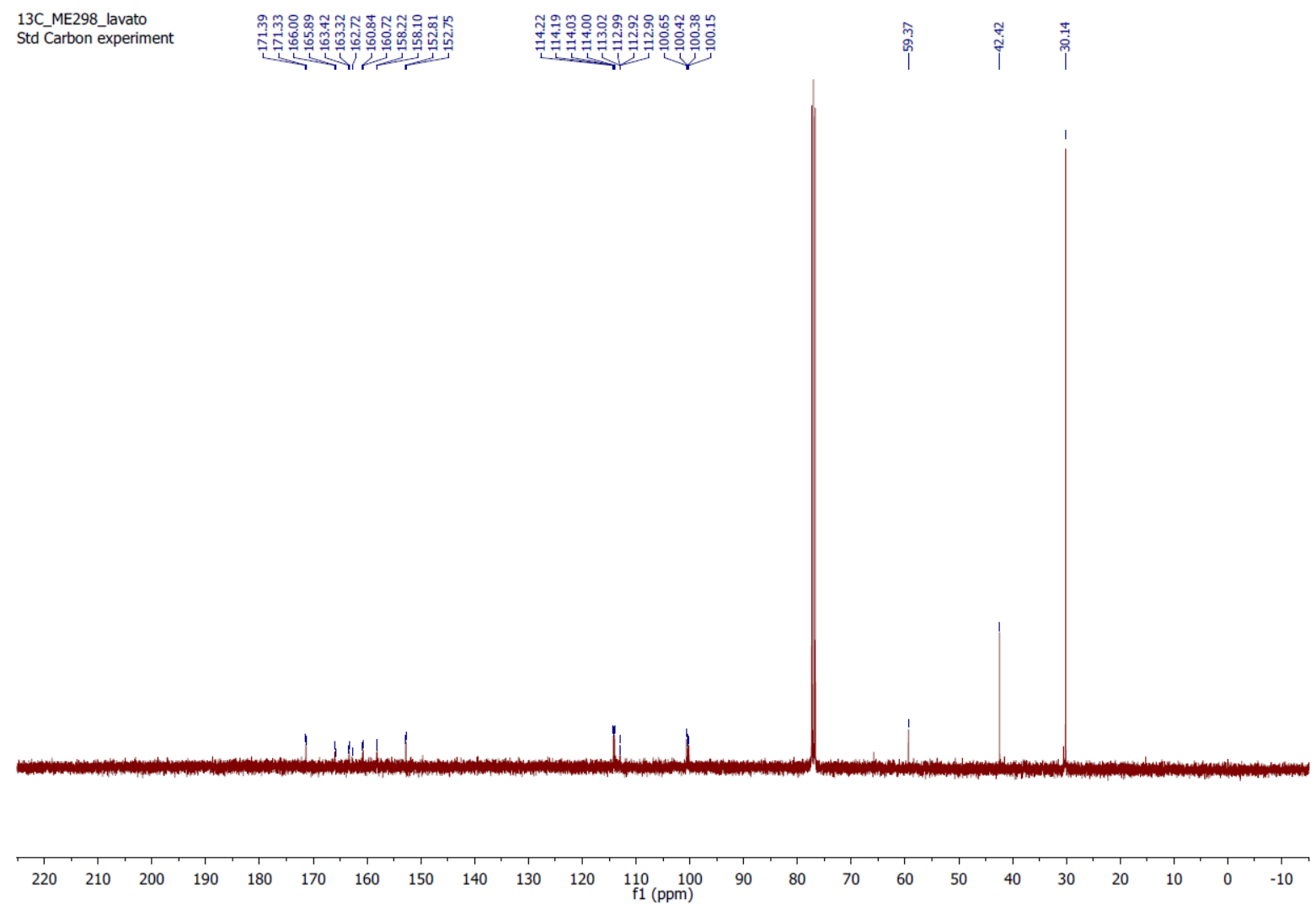

Figure S7b. ${ }^{13} \mathrm{C}$ NMR spectrum of complex 5F. 


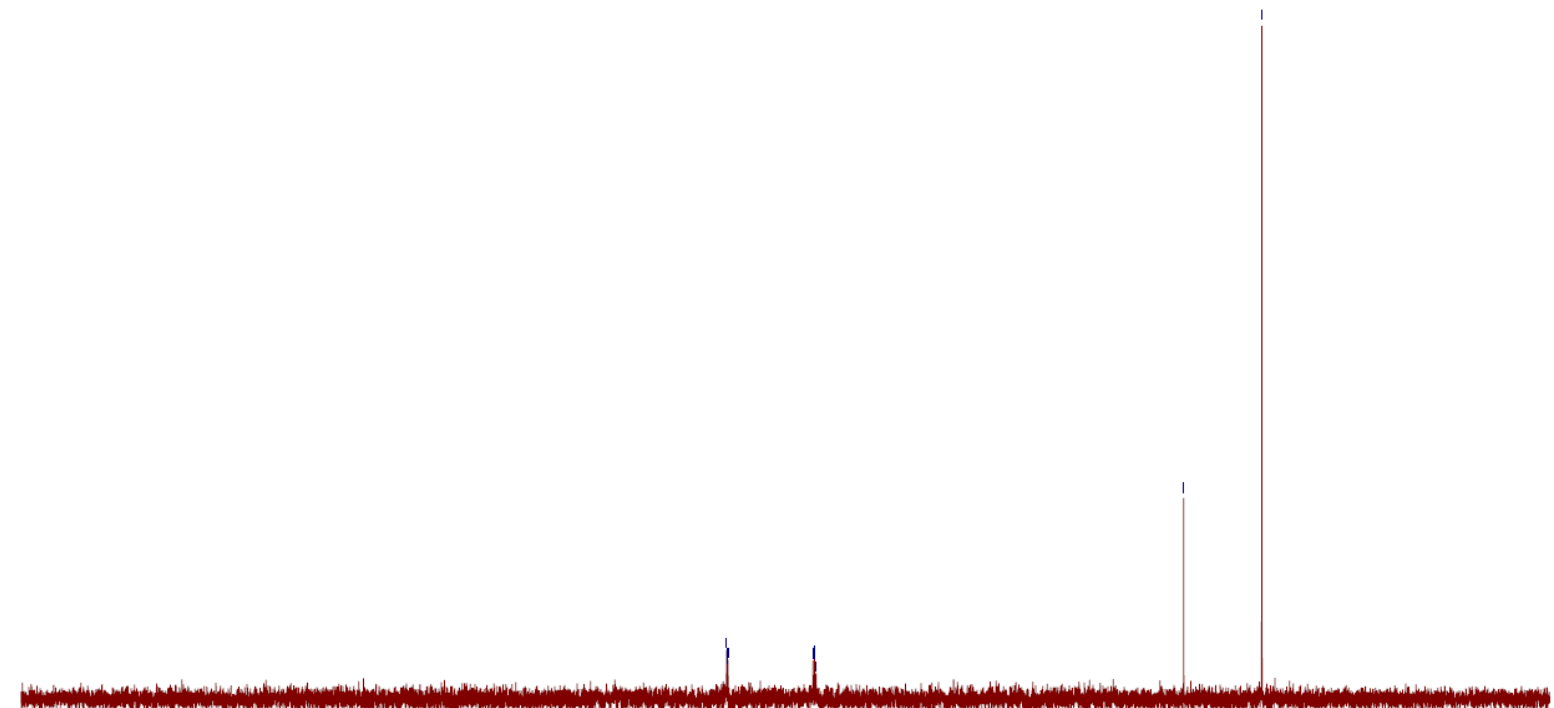

$\begin{array}{llllllllllll}220 & 210 & 200 & 190 & 180 & 170 & 160 & 150 & 140 & 130 & 120 & 110 \\ \mathrm{f} 1(\mathrm{ppm}) & 100\end{array}$

Figure S7c. DEPT NMR spectrum of complex 5F.

19F_ME298_doposcambio Std Fluorine

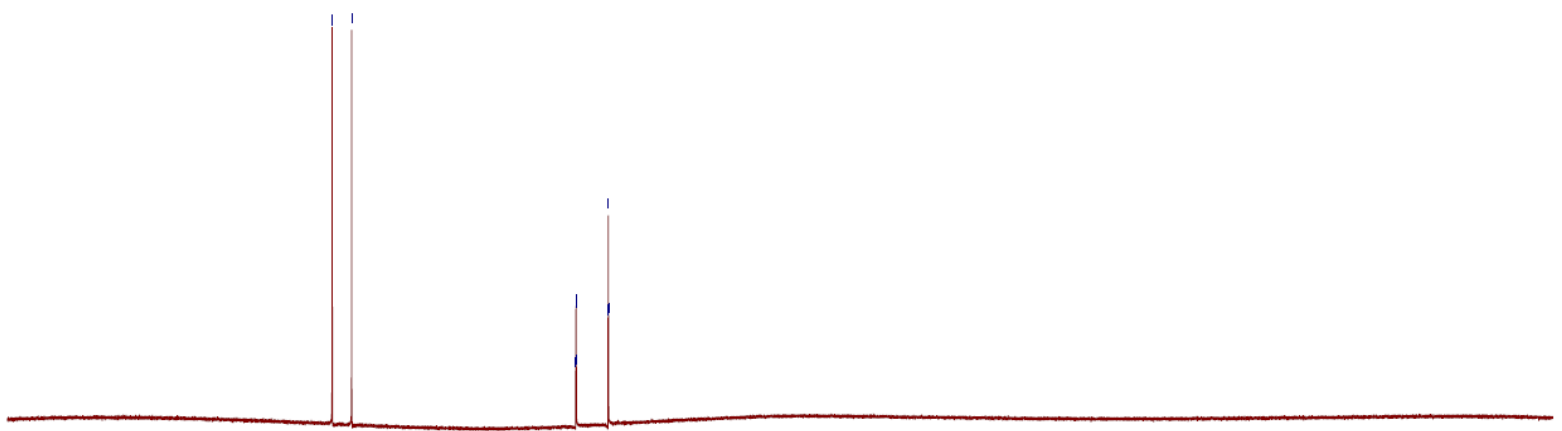

Figure S7d. ${ }^{19} \mathrm{~F}$ NMR spectrum of complex 5F. 


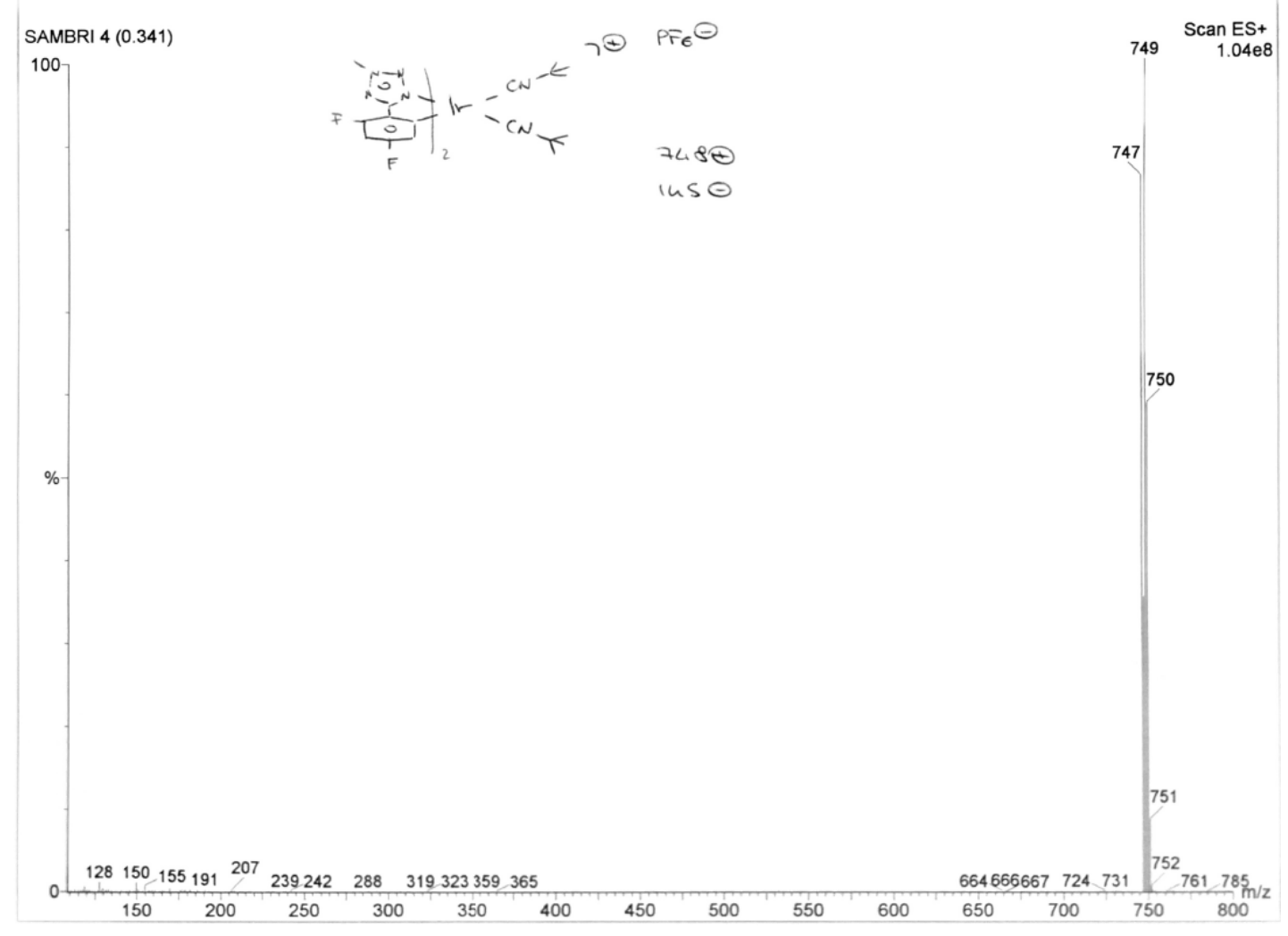

Figure S7e. ESI ${ }^{+}$spectrum of complex 5F.

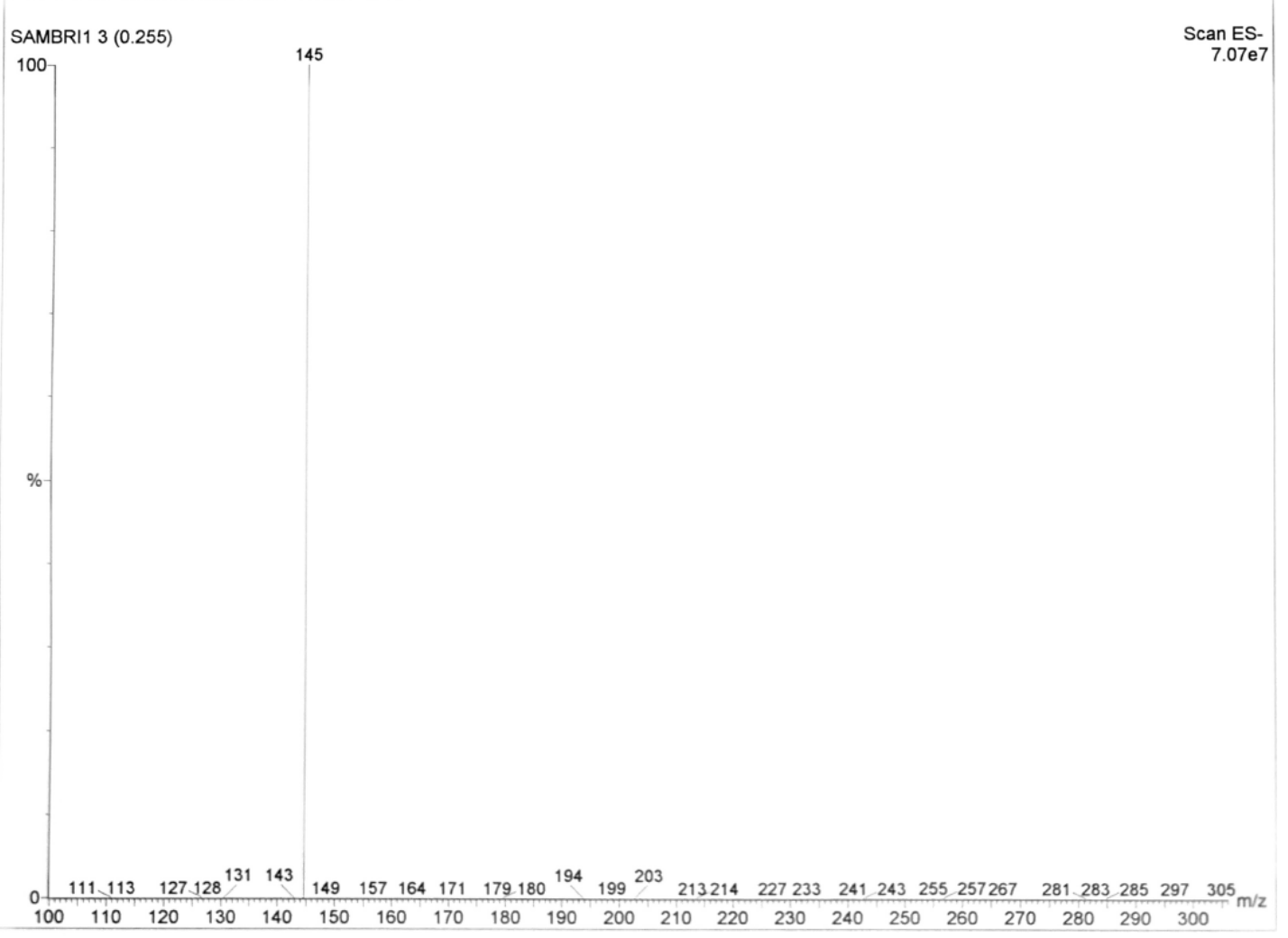

Figure S7f. ESI- spectrum of complex 5F. 
Table S1. Crystallographic parameters for complexes $2 \mathrm{~F}$ and $4 \mathrm{~F}$.

\begin{tabular}{|c|c|c|}
\hline & $2 F$ & $4 \mathrm{~F}$ \\
\hline Formula & $\mathrm{C}_{34} \mathrm{H}_{34} \mathrm{~N}_{10} \mathrm{~F}_{10} \mathrm{PIr}$ & $\mathrm{C}_{36} \mathrm{H}_{38} \mathrm{~N}_{12} \mathrm{O}_{3} \mathrm{~F}_{7} \mathrm{Ir}$ \\
\hline $\mathrm{F}_{\mathrm{w}}$ & 995.88 & 1011.98 \\
\hline $\mathrm{T}, \mathrm{K}$ & 298 & 298 \\
\hline$\lambda, \AA$ & $0.71073(\mathrm{Mo}-\mathrm{K} \alpha)$ & $0.71073(\mathrm{Mo}-\mathrm{K} \alpha)$ \\
\hline Crystal system & Monoclinic & Monoclinic \\
\hline Space group & $\mathrm{P} 2_{1} / \mathrm{c}$ & $\mathrm{P} 2_{1} / \mathrm{n}$ \\
\hline a, $\AA$ & $10.9234(13)$ & $14.957(2)$ \\
\hline $\mathrm{b}, \AA$ & $19.252(2)$ & $14.2926(19)$ \\
\hline$c, \AA$ & $17.806(2)$ & $18.831(3)$ \\
\hline$\alpha, \operatorname{deg}$ & 90.000 & 90.000 \\
\hline$\beta$, deg & $97.958(2)$ & $95.050(2)$ \\
\hline$\gamma, \operatorname{deg}$ & 90.000 & 90.000 \\
\hline Cell volume, $\AA^{3}$ & $3708.6(8)$ & $4010.2(9)$ \\
\hline $\mathrm{Z}$ & 4 & 4 \\
\hline $\mathrm{D}_{\mathrm{c}}, \mathrm{g} \mathrm{cm}^{-3}$ & 1.784 & 1.676 \\
\hline$\mu, \mathrm{mm}^{-1}$ & 3.735 & 3.414 \\
\hline $\mathrm{F}(000)$ & 1960 & 2008 \\
\hline $\mathrm{h}, \mathrm{k}, 1 \mathrm{max}$ & $\begin{array}{l}-13<\mathrm{h}<9,-22<\mathrm{k}<19, \\
-21<\mathrm{l}<21\end{array}$ & $\begin{array}{l}-18<\mathrm{h}<18,-17<\mathrm{k}<17, \\
-23<\mathrm{l}<23\end{array}$ \\
\hline Crystal size, $\mathrm{mm}$ & $0.25,0.15,0.15$ & $0.20,0.18,0.15$ \\
\hline$\theta$ limits, $^{\circ}$ & 1.57 to 26.00 & 1.67 to 26.00 \\
\hline Reflections collected & 7073 & 7884 \\
\hline Independent reflections & 5922 & 5965 \\
\hline Data/restraints/param. & $5922 / 0 / 513$ & $5965 / 0 / 540$ \\
\hline Weight. Scheme & $0.0520,12.788$ & $0.0398,13.4608$ \\
\hline GOF on $\mathrm{F}^{2}$ & 1.028 & 0.982 \\
\hline $\mathrm{R}_{1}(\mathrm{I}>4 \sigma(\mathrm{I}))$ & 0.0366 & 0.0418 \\
\hline $\mathrm{wR}_{2}$ (all data) & 0.0975 & 0.1059 \\
\hline Peak/hole, e $\AA^{-3}$ & 2.502 and -1.999 & 1.691 and -0.598 \\
\hline CCDC & 2011527 & 2011528 \\
\hline NOTES & - & $\begin{array}{l}\text { The cell contains a molecule of } \\
\text { solvent (diethyl ether) and a } \\
\text { disordered trifluoroacetate anion. }\end{array}$ \\
\hline
\end{tabular}




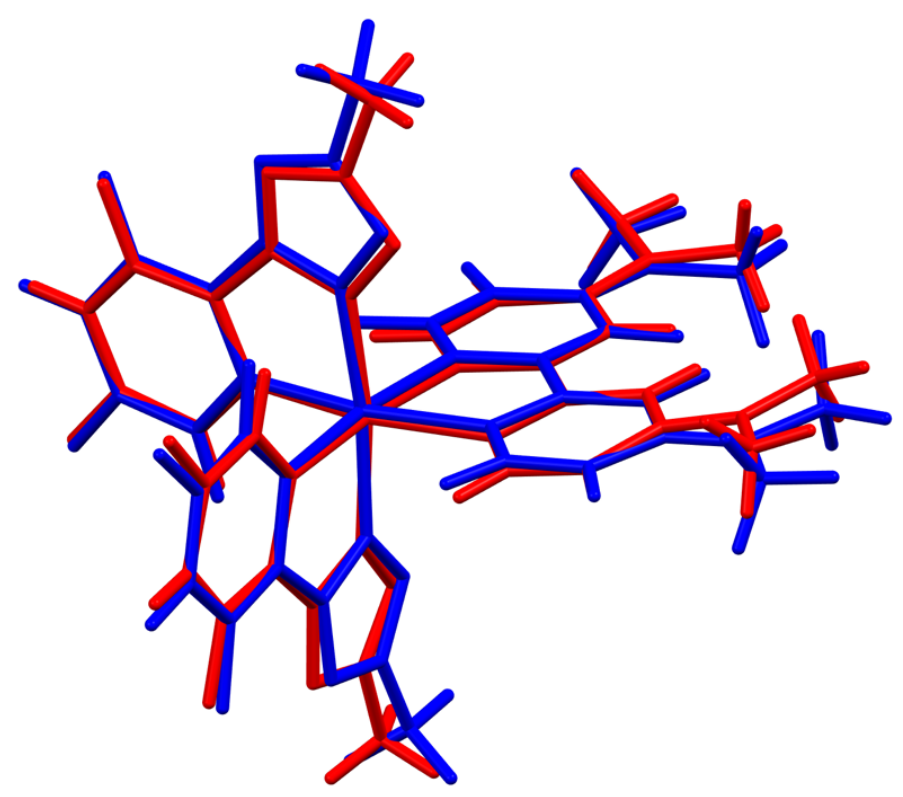

Figure S8. Structural overlay ( $\mathrm{H}$ atoms omitted) between the experimental X-ray structure of the cationic part of $4 \mathrm{~F}$ (red) and the theoretically computed one (blue). The structural overlay is calculated by minimizing the root-mean-square deviation $(\mathrm{RMSD})$ of all the atomic positions (RMSD $=0.226 \AA$ ).
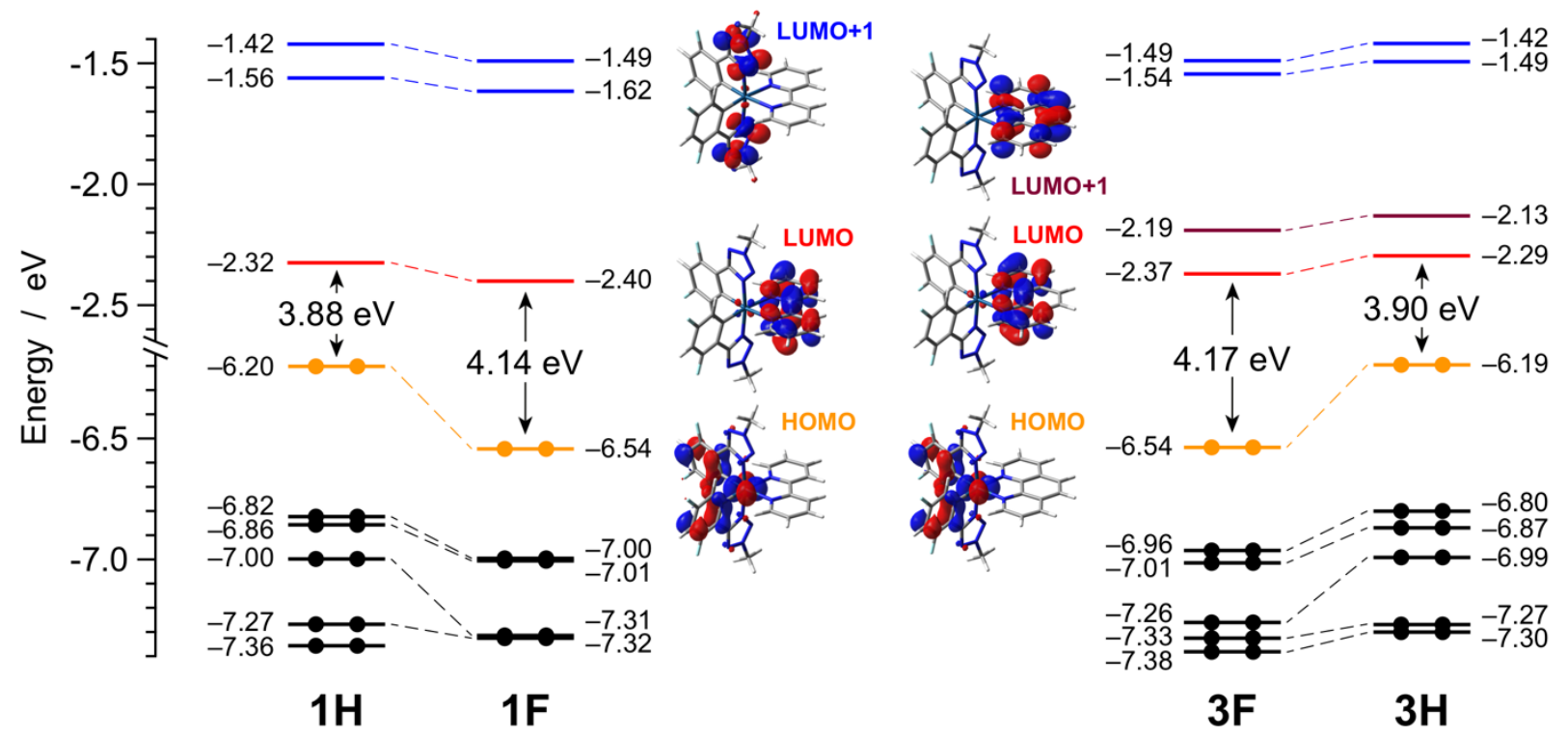

Figure S9. Energy diagram comparing the energy values of the frontier Kohn-Sham molecular orbitals of two selected complexes of the present Ir-dfptrz series (i.e., 1F and 3F) with the fluorine-free analogues of the previously published Ir-ptrz series (i.e., $\mathbf{1} \mathbf{H}$ and $\mathbf{3 H}$ ). Calculations are carried out in acetonitrile, using PCM. For some relevant orbitals, the corresponding isosurface is also displayed for the sake of clarity (isovalue = $\left.0.04 \mathrm{e}^{1 / 2} \mathrm{bohr}^{-3 / 2}\right)$. Relevant orbitals with similar topology are plotted with the same color for an easier comparison. 


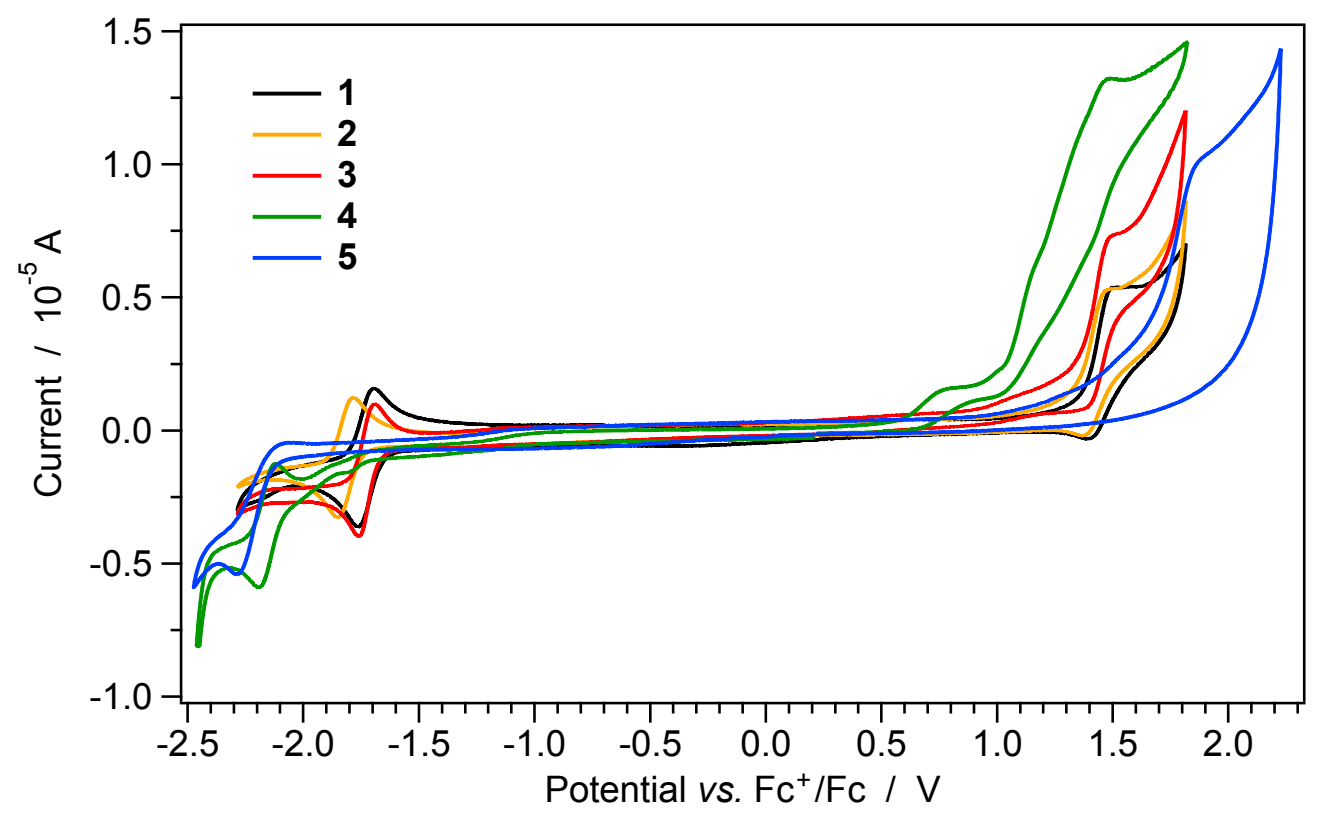

Figure S10. Cyclic voltammograms of complexes $1 F-5 F(0.5 \mathrm{mM})$ in room-temperature acetonitrile solution (with $0.1 \mathrm{M} \mathrm{TBAPF}_{6}$ ) recorded at a scan rate of $100 \mathrm{mV} \mathrm{s}^{-1}$.

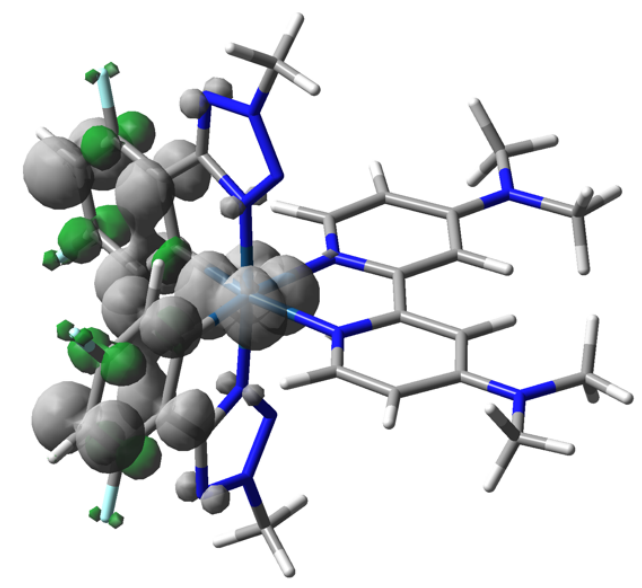

Figure S11. Spin-density distribution of the oxidized radical of complexes 4F in its fully-optimized geometry, computed in acetonitrile (isovalues: $0.002 \mathrm{e} \mathrm{bohr}^{-3}$ ). The topology of the spin-density distribution resembles the one of the HOMO of $\mathbf{4 F}$ indicating that, also in this case, the oxidation process can be attributable to the formal oxidation of iridium(III) to iridium(IV). 


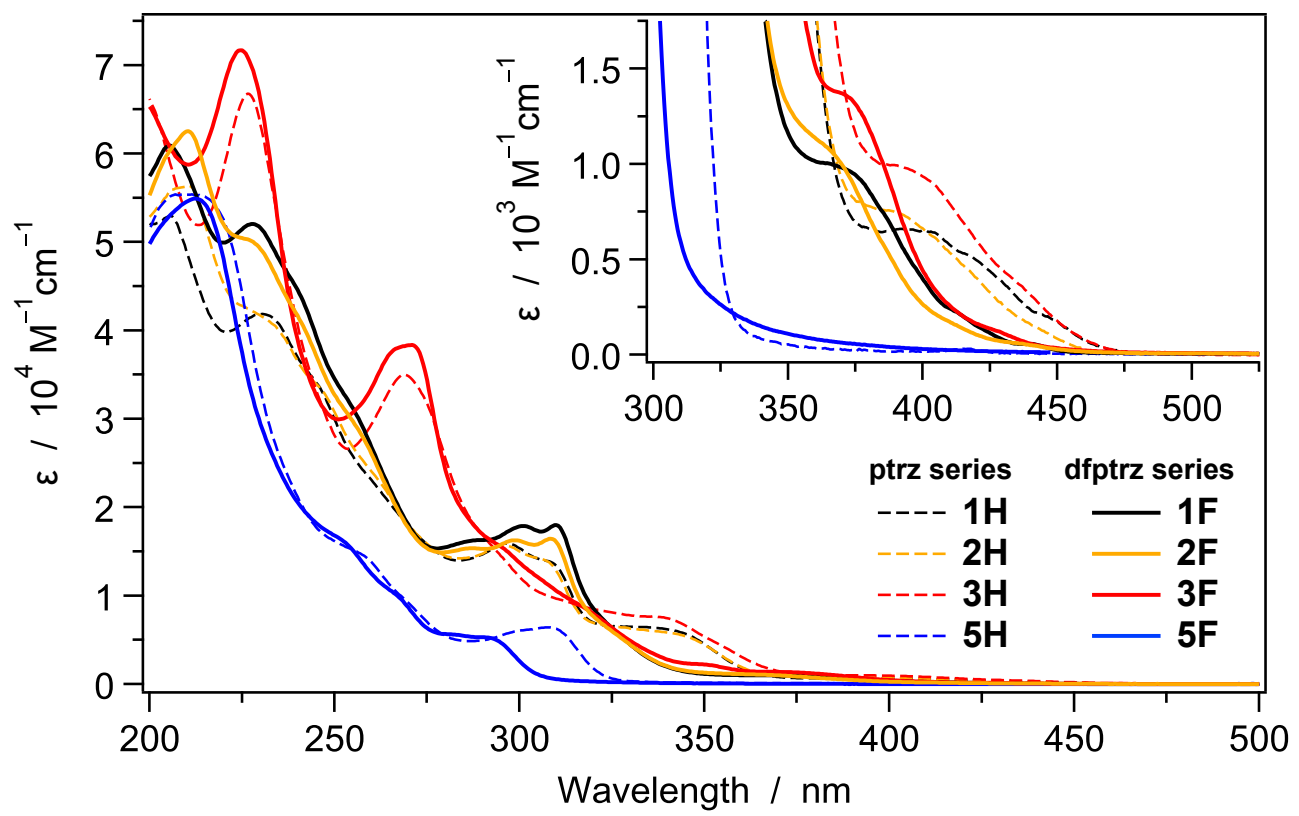

Figure S12. Comparison between the absorption spectra of the present Ir-dfptrz series (i.e., 1F-3F and 5F) and the ones of the previously published fluorine-free analogues (i.e., $1 \mathbf{H}-\mathbf{3 H}$ and $\mathbf{5 H}$ ). All spectra are recorded in room-temperature acetonitrile solution. The lower-energy transitions are magnified in the inset.

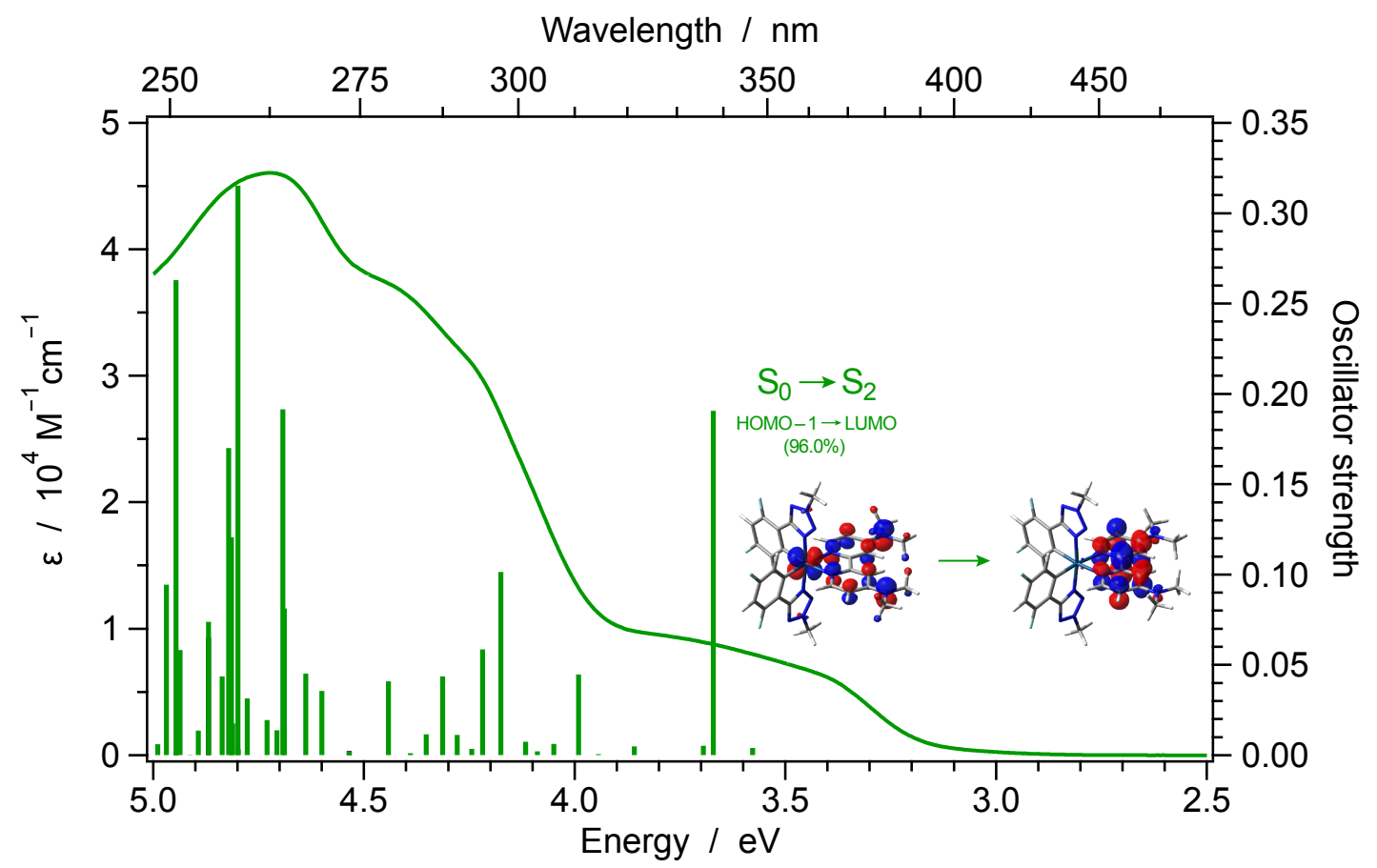

Figure S13. First 50 singlet vertical excitations (green bars) computed for 4F in acetonitrile, using PCM and the linear-response formalism. Theoretical data are compared with the corresponding room-temperature experimental absorption spectrum (green line) in the same solvent. 
Table S2a. Calculated NTOs couples describing the first five triplet excitations for complex 1F in acetonitrile (see Experimental Section for further details). The $\lambda$ value is the natural transition orbital eigenvalue associated with each NTOs couple; orbital isovalue: $0.04 \mathrm{e}^{-1 / 2} \mathrm{bohr}^{-3 / 2}$.

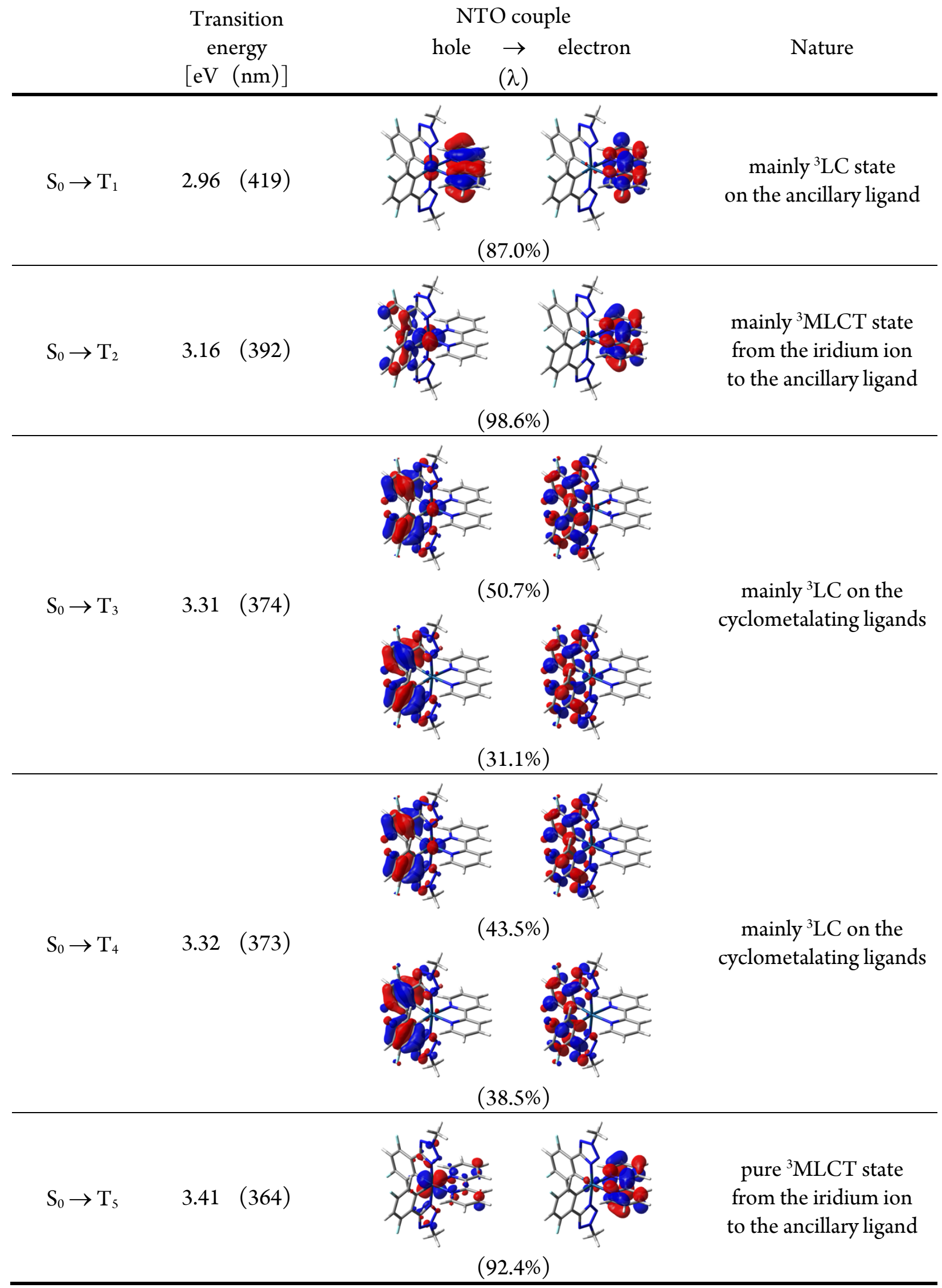


Table S2b. Calculated NTOs couples describing the first five triplet excitations for complex 2F in acetonitrile (see Experimental Section for further details). The $\lambda$ value is the natural transition orbital eigenvalue associated with each NTOs couple; orbital isovalue: $0.04 \mathrm{e}^{-1 / 2} \mathrm{bohr}^{-3 / 2}$.

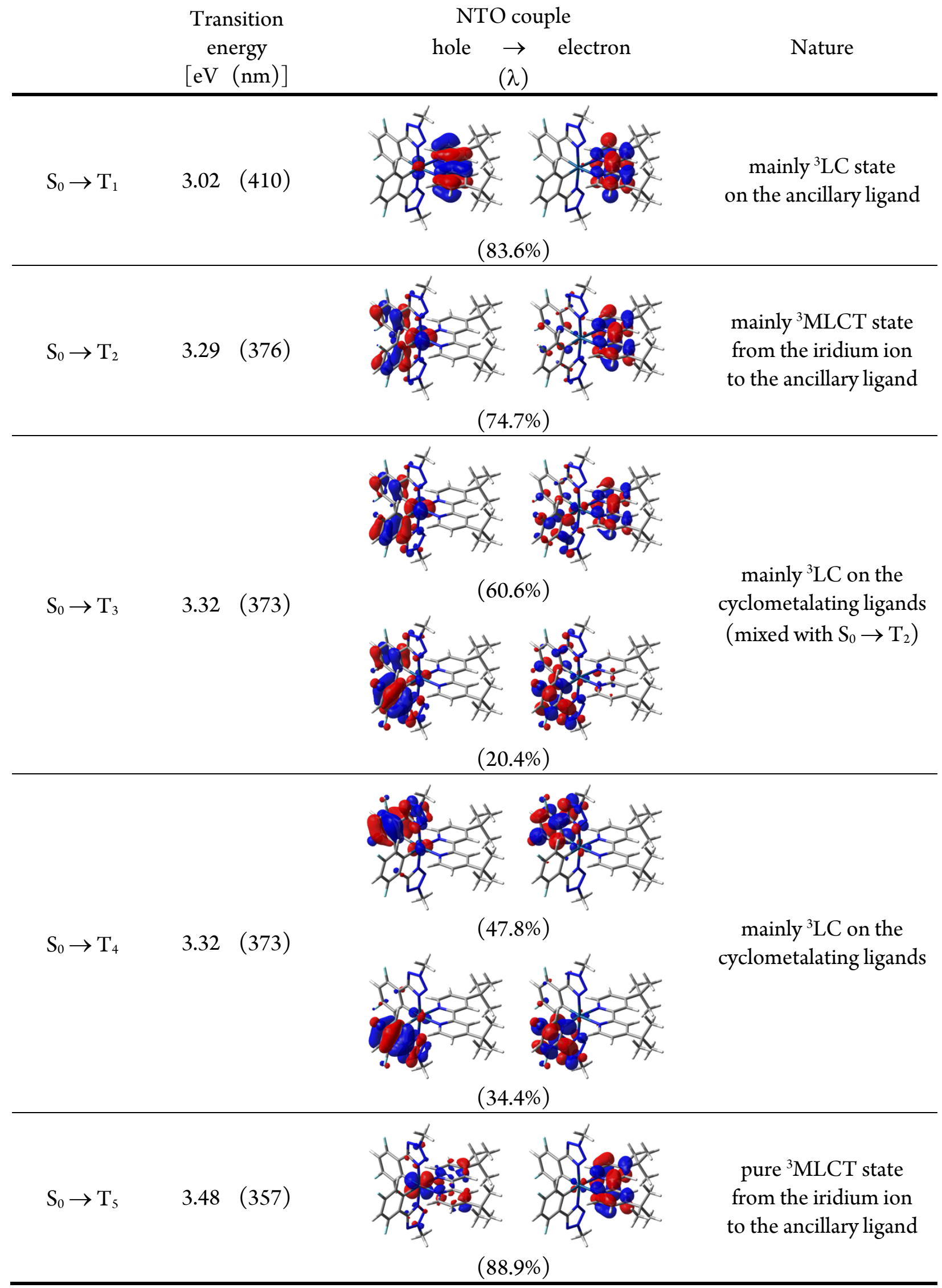


Table S2c. Calculated NTOs couples describing the first five triplet excitations for complex 3F in acetonitrile (see Experimental Section for further details). The $\lambda$ value is the natural transition orbital eigenvalue associated with each NTOs couple; orbital isovalue: $0.04 \mathrm{e}^{-1 / 2} \mathrm{bohr}^{-3 / 2}$.

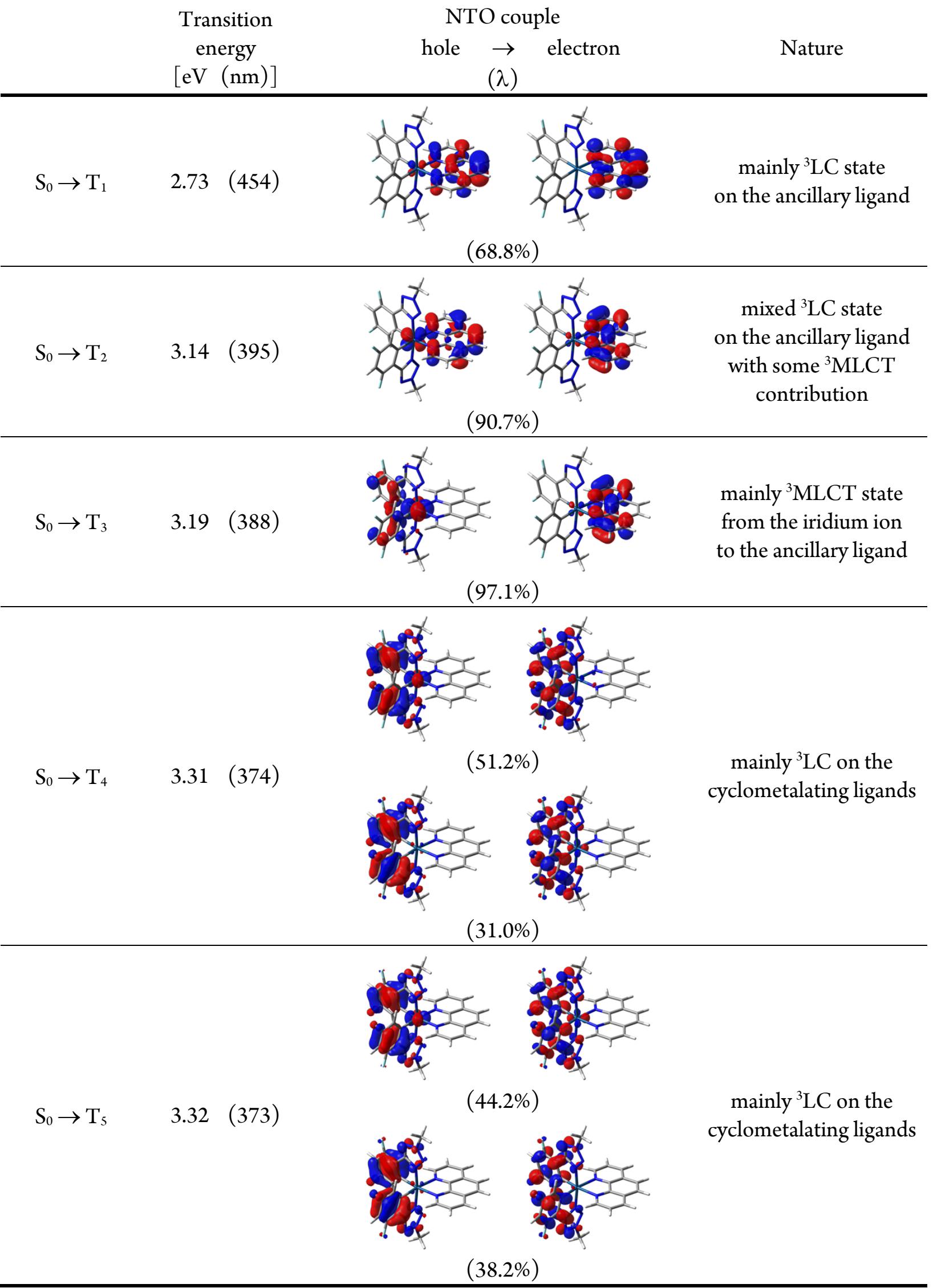


Table S2d. Calculated NTOs couples describing the first six triplet excitations for complex 4F in acetonitrile (see Experimental Section for further details). The $\lambda$ value is the natural transition orbital eigenvalue associated with each NTOs couple; orbital isovalue: $0.04 \mathrm{e}^{-1 / 2} \mathrm{bohr}^{-3 / 2}$.

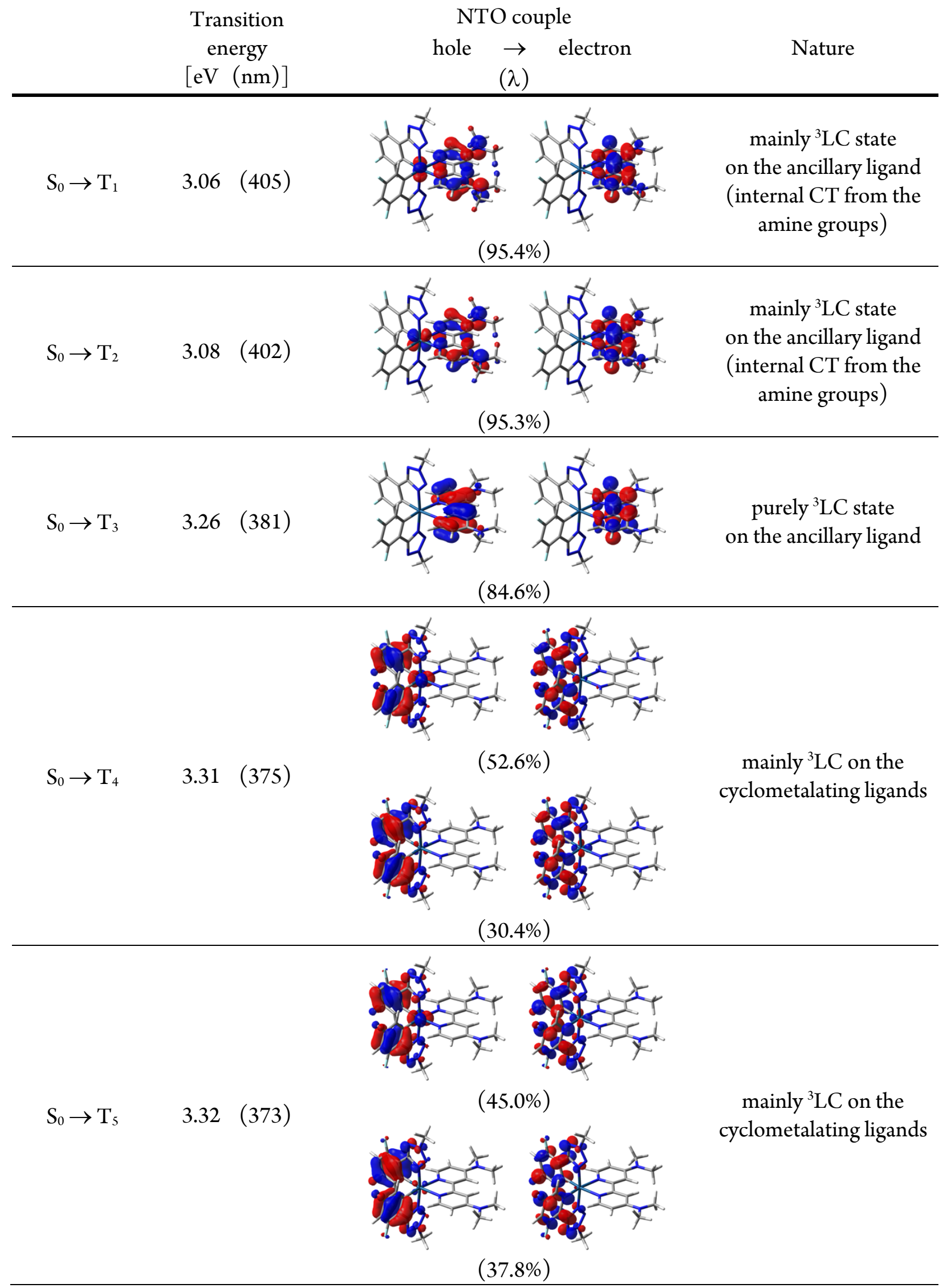


$\mathrm{S}_{0} \rightarrow \mathrm{T}_{6}$
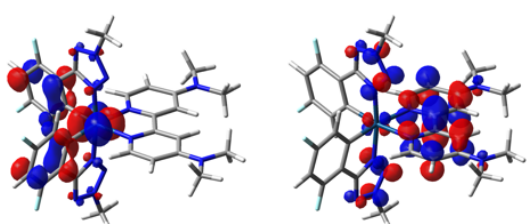

mainly ${ }^{3} \mathrm{MLCT}$ state

from the iridium ion to the ancillary ligand

$(87.1 \%)$

Table S2e. Calculated NTOs couples describing the first four triplet excitations for complex $5 F$ in acetonitrile (see Experimental Section for further details). The $\lambda$ value is the natural transition orbital eigenvalue associated with each NTOs couple; orbital isovalue: $0.04 \mathrm{e}^{-1 / 2} \mathrm{bohr}^{-3 / 2}$.

energy
$[\mathrm{eV}(\mathrm{nm})]$


Table S2f. Calculated NTOs couples describing the first five triplet excitations for complex $1 \mathrm{H}$ in acetonitrile (see Experimental Section for further details). The $\lambda$ value is the natural transition orbital eigenvalue associated with each NTOs couple; orbital isovalue: $0.04 \mathrm{e}^{-1 / 2} \mathrm{bohr}^{-3 / 2}$.

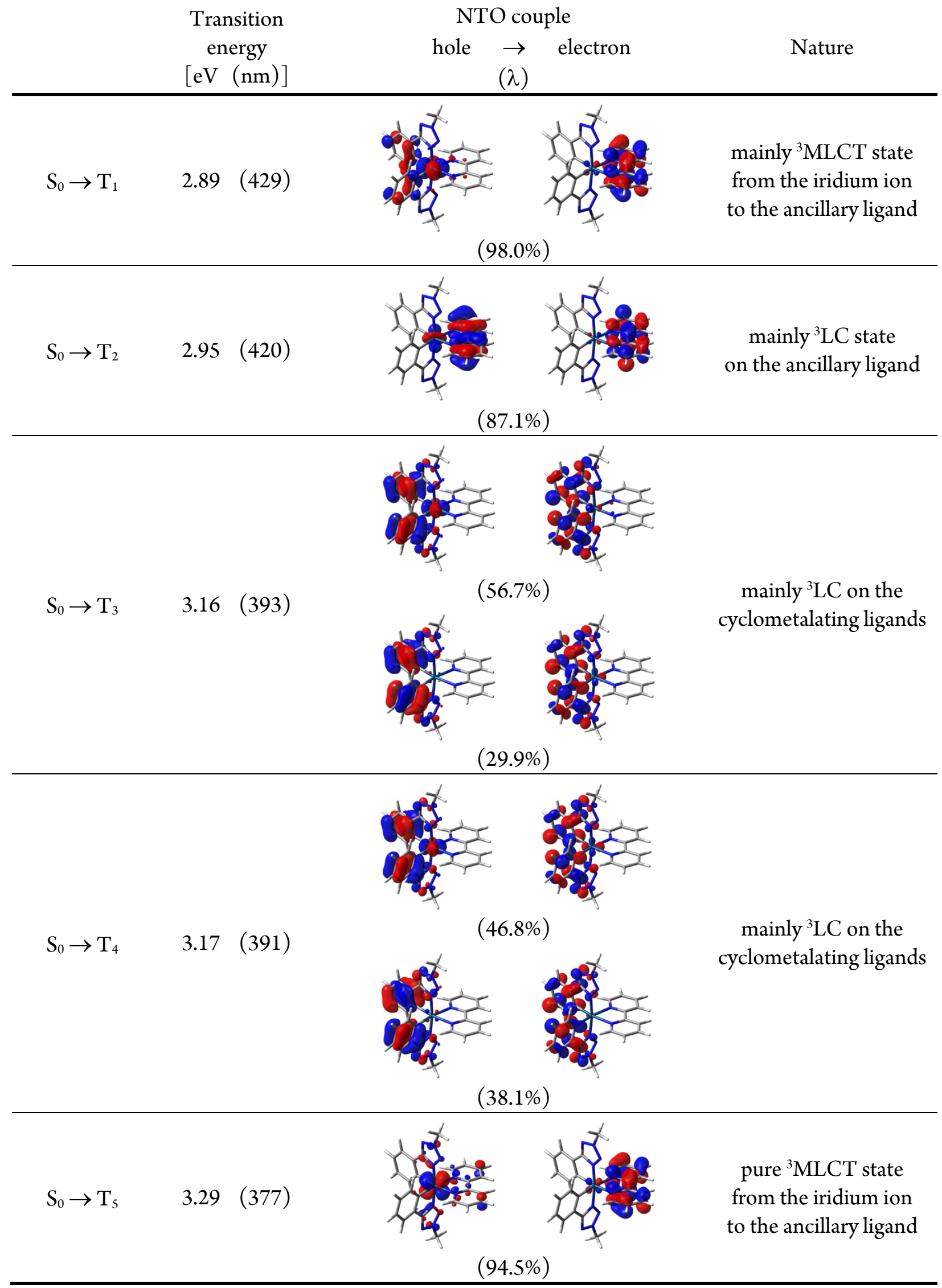


Table S2g. Calculated NTOs couples describing the first five triplet excitations for complex $3 \mathrm{H}$ in acetonitrile (see Experimental Section for further details). The $\lambda$ value is the natural transition orbital eigenvalue associated with each NTOs couple; orbital isovalue: $0.04 \mathrm{e}^{-1 / 2} \mathrm{bohr}^{-3 / 2}$.

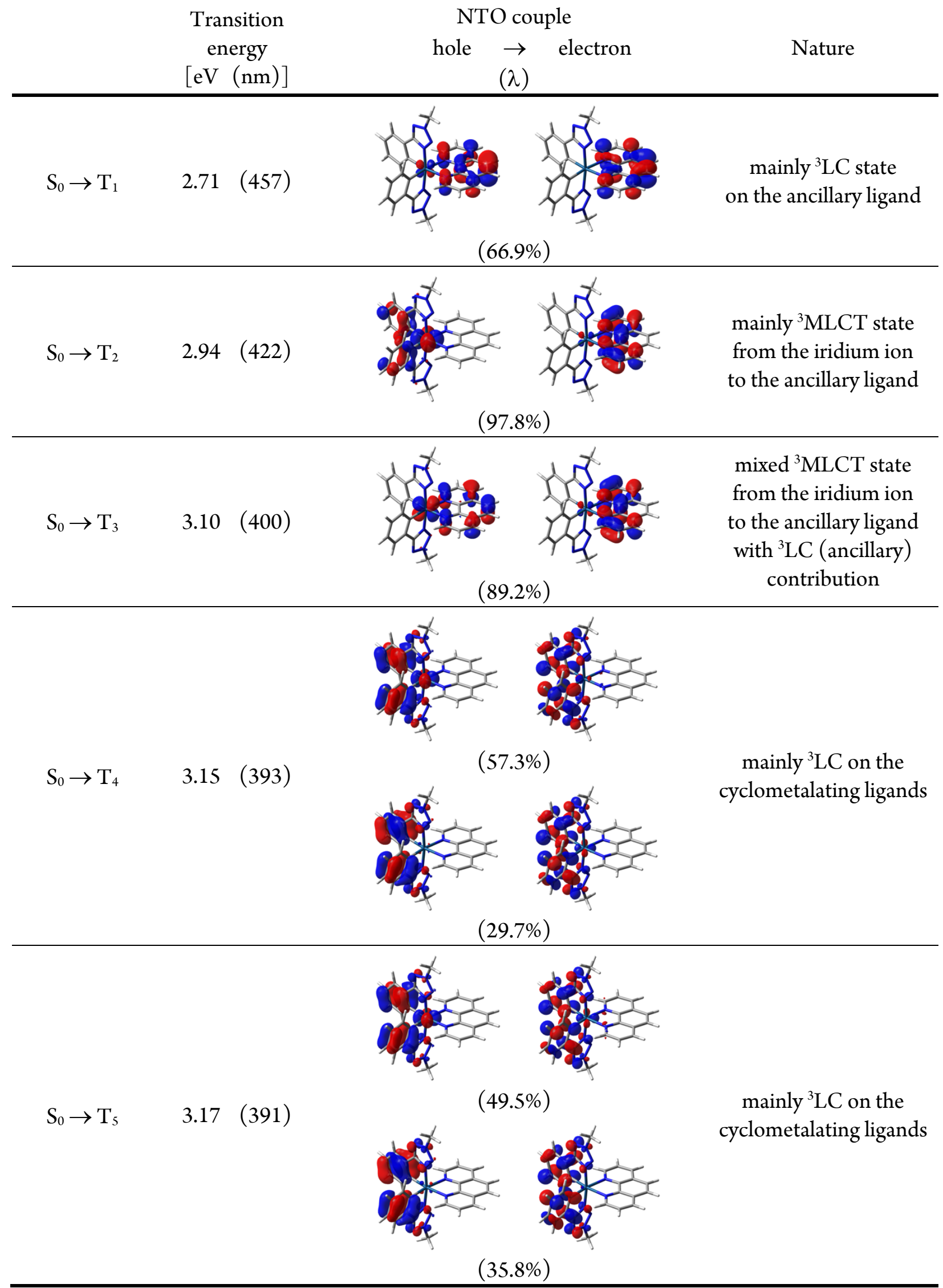


Table S2h. Calculated NTOs couples describing the first four triplet excitations for complex $5 \mathrm{H}$ in acetonitrile (see Experimental Section for further details). The $\lambda$ value is the natural transition orbital eigenvalue associated with each NTOs couple; orbital isovalue: $0.04 \mathrm{e}^{-1 / 2} \mathrm{bohr}^{-3 / 2}$.

energy
$[\mathrm{eV}(\mathrm{nm})]$




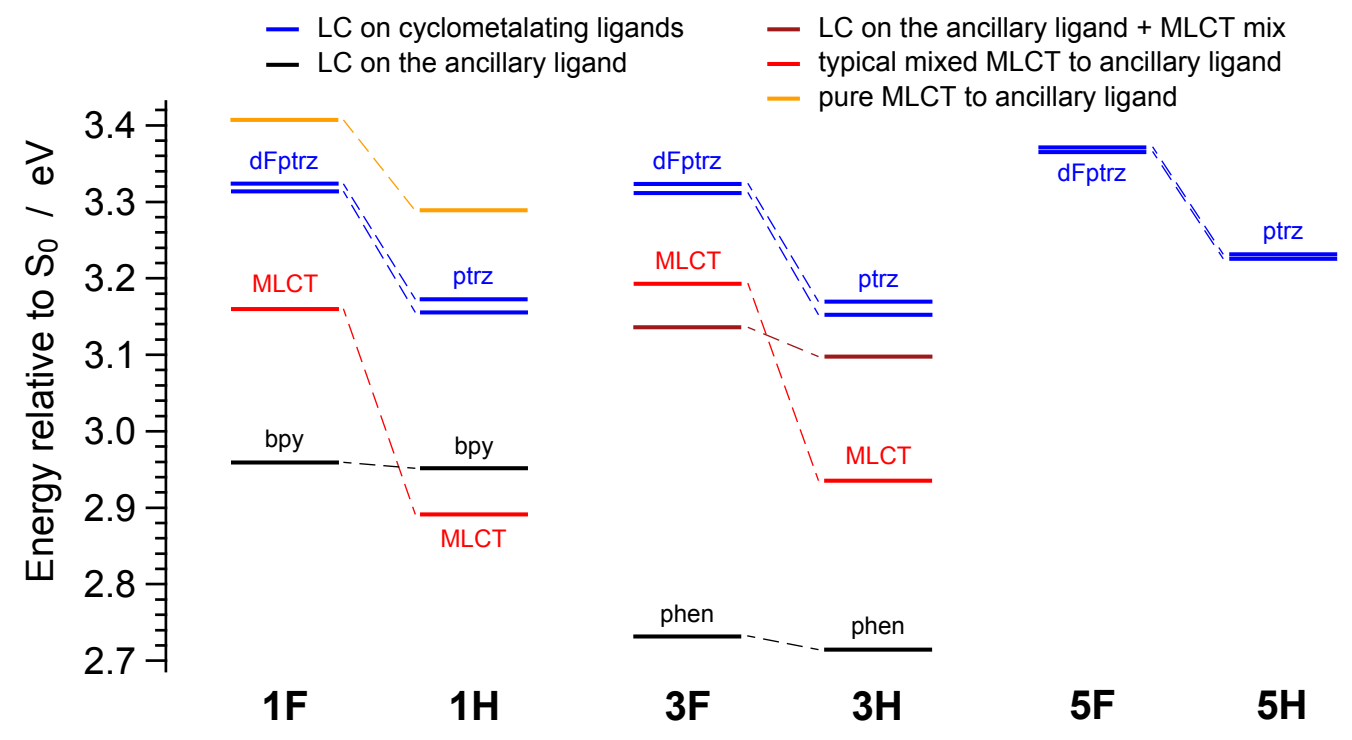

Figure S14. Comparison between the lowest-lying triplet states of some selected complexes of the present Irdfptrz series (i.e., $1 \mathrm{~F}, \mathbf{3 F}$ and $\mathbf{5 F}$ ) and the analogues of the previously published fluorine-free one (i.e., $1 \mathrm{H}, 3 \mathrm{H}$ and $\mathbf{5 H}$ ). All data are computed in acetonitrile as vertical excitations from the respective ground-state minimum-energy geometries.

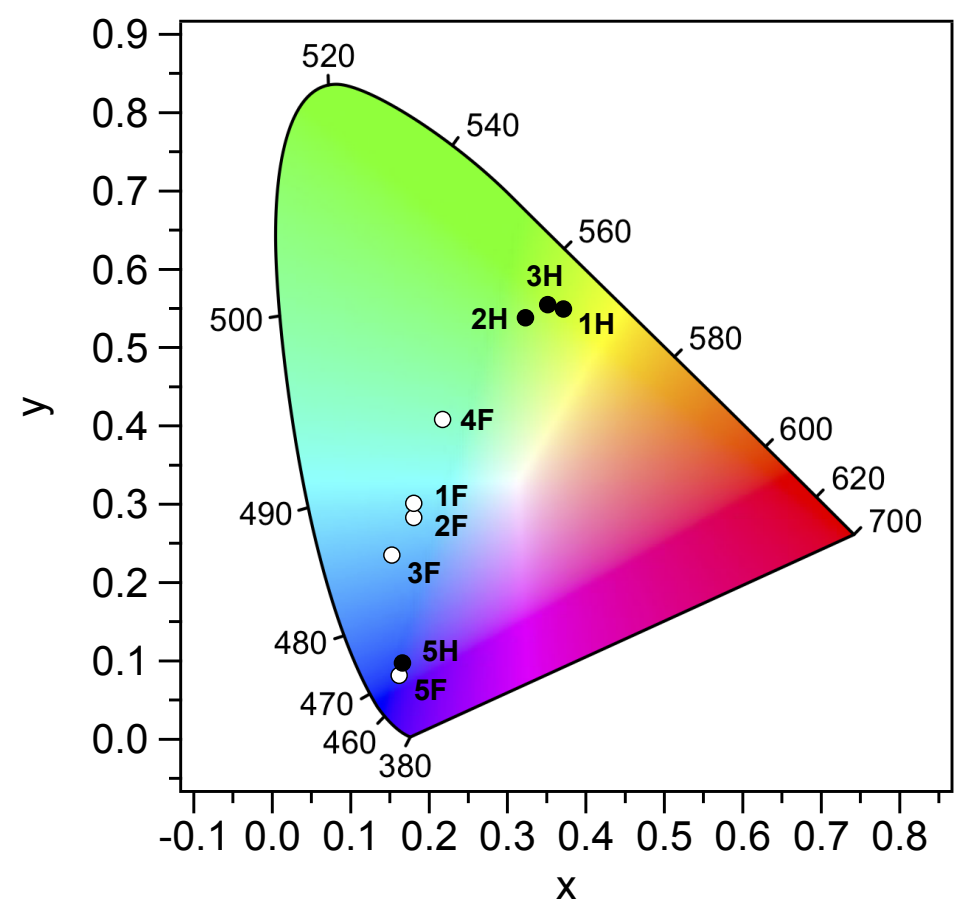

Figure S15. CIE-1931 chromaticity diagram reporting the emission color of the present Ir-dfptrz series (i.e., $\mathbf{1 F}-\mathbf{5 F}$ ) and of the previously published fluorine-free one (i.e., $1 \mathbf{H}-3 \mathrm{H}$ and $\mathbf{5 H})$ in acetonitrile at $298 \mathrm{~K}$. 


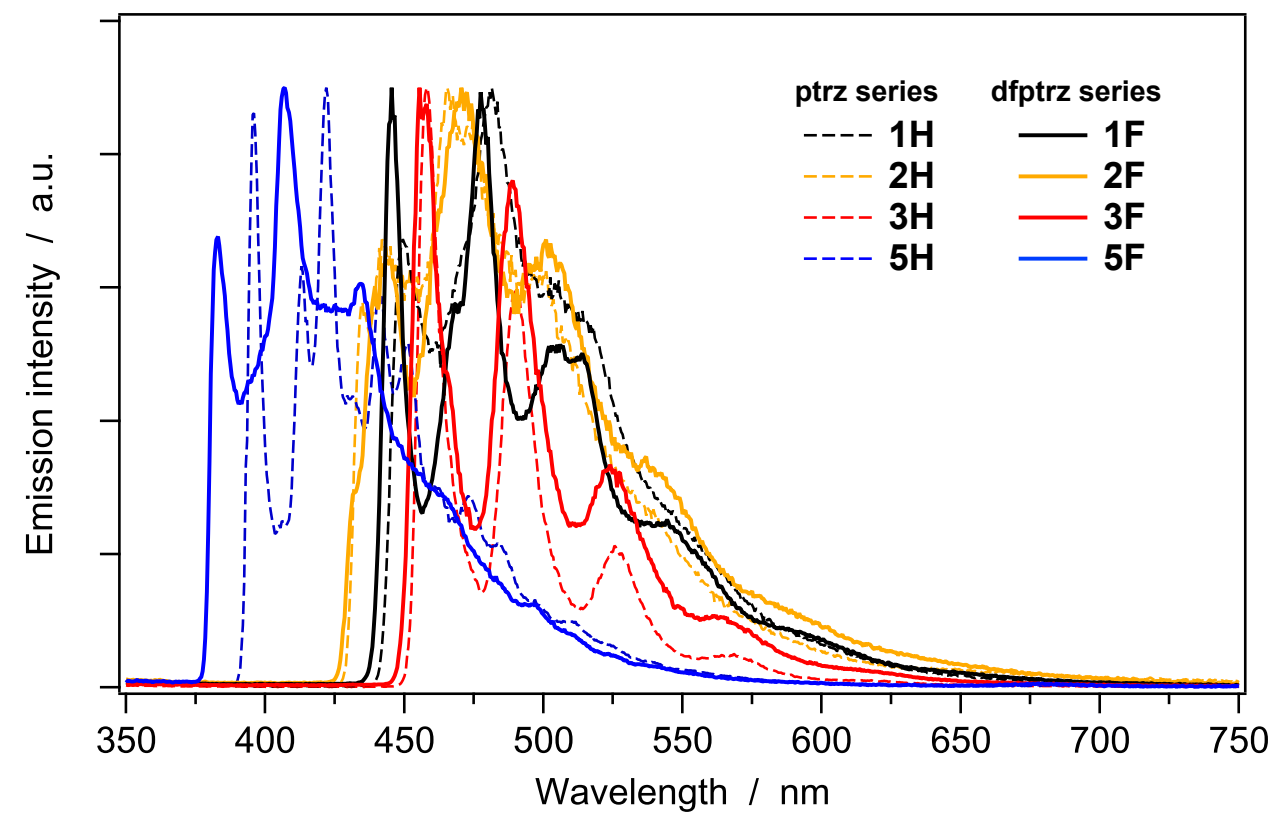

Figure S16. Comparison between the 77-K emission spectra of the present Ir-dfptrz series (i.e., $1 \mathrm{~F}-3 \mathrm{~F}$ and $\mathbf{5 F}$ ) and the ones of the previously published fluorine-free analogues (i.e., $1 \mathrm{H}-3 \mathrm{H}$ and $\mathbf{5 H}$ ).

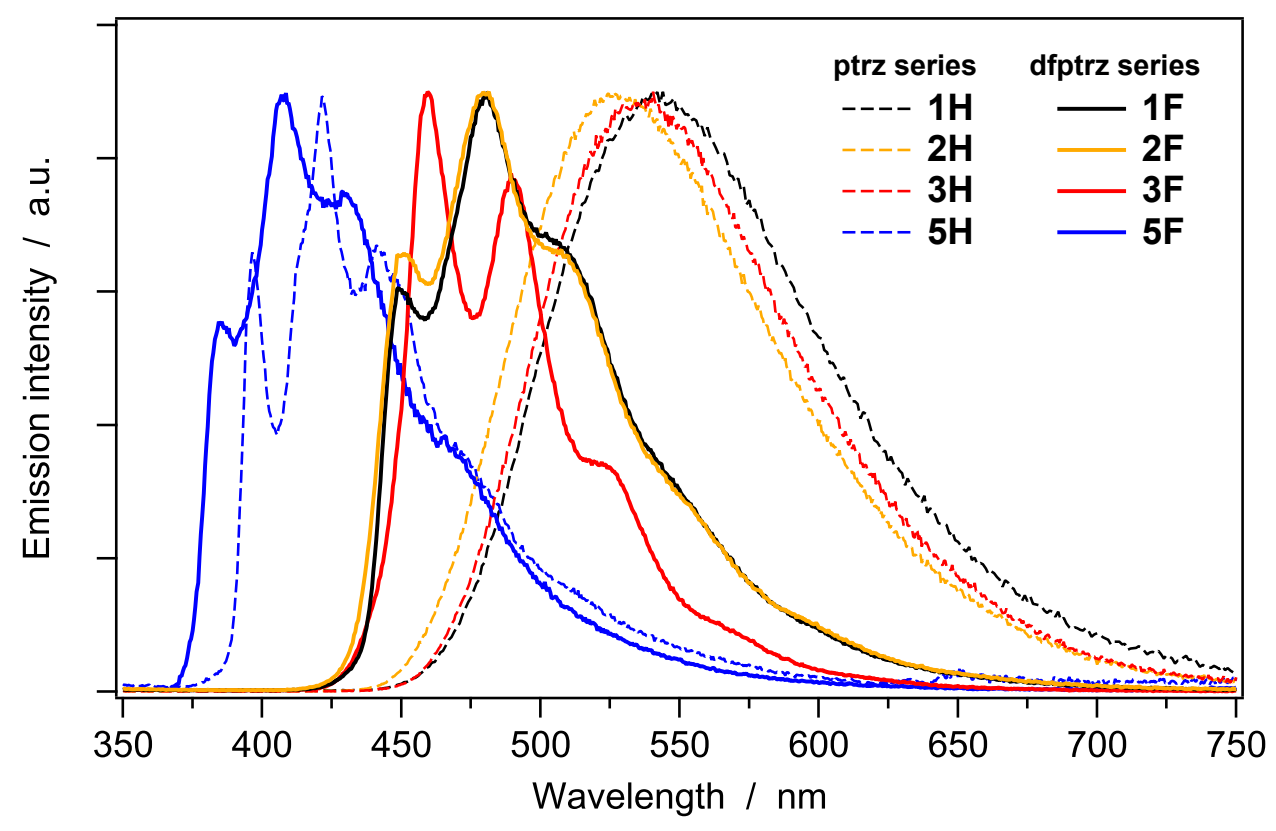

Figure S17. Comparison between the room-temperature emission spectra of the present Ir-dfptrz series (i.e., $\mathbf{1 F}-\mathbf{3 F}$ and $\mathbf{5 F}$ ) and the ones of the previously published fluorine-free analogues (i.e., $\mathbf{1} \mathbf{H}-\mathbf{3 H}$ and $\mathbf{5 H}$ ). 

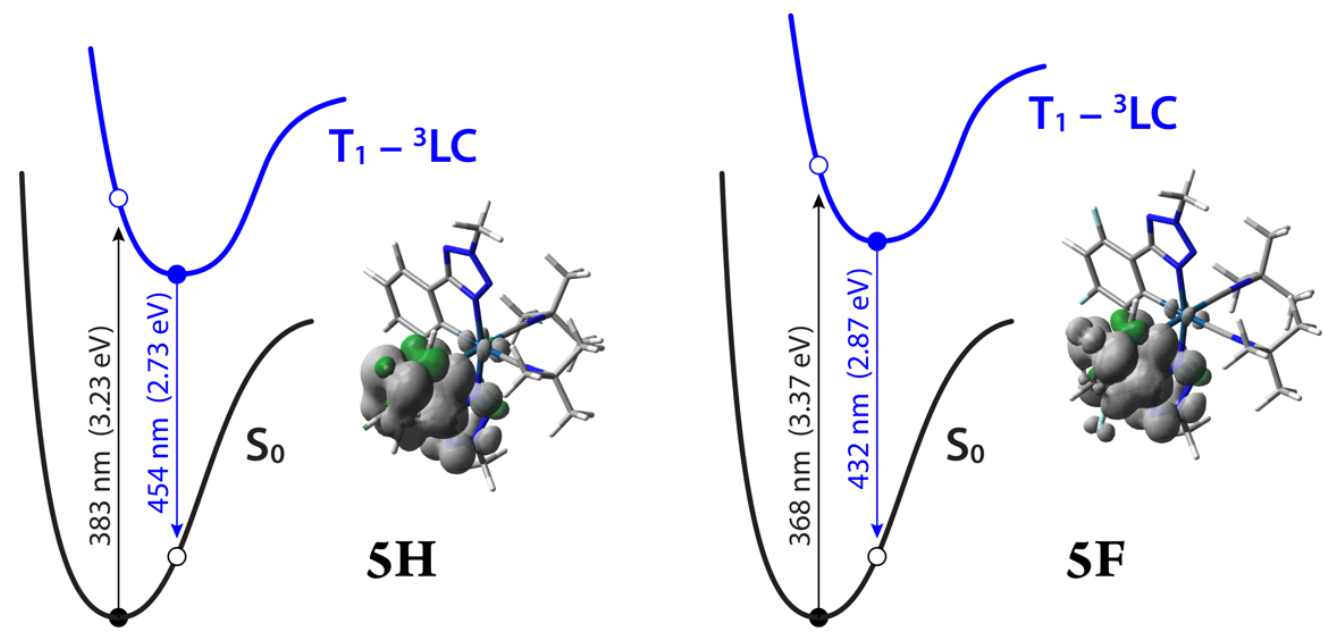

Figure S18. Schematic energy diagram reporting the ground state $\left(S_{0}\right)$ and the lowest triplet state $\left({ }^{3} \mathrm{LC}\right)$ for the isocyanide complexes $\mathbf{5 H}$ (left) and $\mathbf{5 F}$ (right). Estimated absorption and emission energies are also reported (not in scale). The unpaired-electron spin-density surfaces calculated the fully-relaxed tripletstate minima are also depicted (isovalue: $0.002 \mathrm{e} \mathrm{bohr}^{-3}$ ).

Table S3. Luminescence properties and photophysical parameters of complexes $1 \mathrm{H}-3 \mathrm{H}$ and $5 \mathrm{H}$ in acetonitrile diluted solution at 298 and $77 \mathrm{~K}$. Data are taken from Inorg. Chem. 2014, 53, 7709-7721.

\begin{tabular}{|c|c|c|c|c|c|c|c|}
\hline & & $\mathrm{CN}$ oxyge & ree sol & n, $298 \mathrm{~K}$ & & $\mathrm{CH}_{3} \mathrm{CN}$ rigid mat & \\
\hline & $\lambda_{\mathrm{em}}{ }^{\mathrm{a}}$ & PLQY $^{a}$ & $\overline{\tau^{b}}$ & $\mathrm{k}_{\mathrm{r}}^{\mathrm{c}}$ & $\mathrm{k}_{\mathrm{nr}}{ }^{\mathrm{d}}$ & $\lambda_{\text {em }}{ }^{a}$ & $\overline{\tau^{b}}$ \\
\hline & {$[\mathrm{nm}]$} & [\%] & {$[\mu \mathrm{s}]$} & {$\left[10^{5} \mathrm{~s}^{-1}\right]$} & {$\left[10^{4} \mathrm{~s}^{-1}\right]$} & {$[\mathrm{nm}]$} & {$[\mu \mathrm{s}]$} \\
\hline $1 \mathrm{H}$ & 545 & 54.8 & 1.22 & 4.49 & 37.0 & $450,482,505$ & 3.7 \\
\hline $2 \mathrm{H}$ & 530 & 67.6 & 1.18 & 5.72 & 27.4 & $442,466,500$ & 3.6 \\
\hline $3 \mathrm{H}$ & 540 & 70.3 & 1.67 & 4.22 & 17.8 & $462,495,530,570$ & 11.2 \\
\hline $5 \mathrm{H}$ & $396,422,442$ & 0.5 & 0.87 & 0.06 & 114 & $396,422,446$ & 106 \\
\hline
\end{tabular}

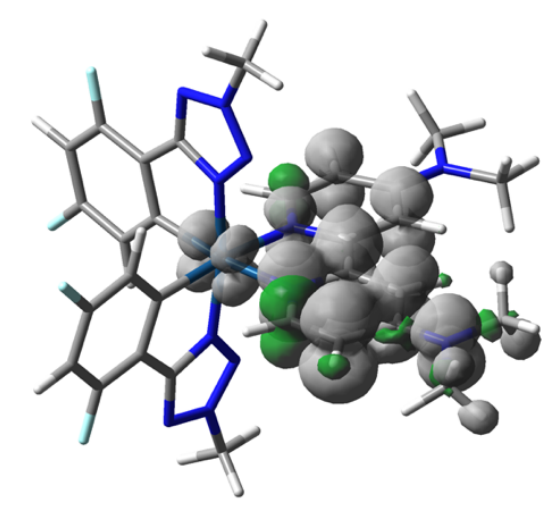

Figure S19. Spin-density distribution of the lowest triplet state of complex $4 \mathrm{~F}$ in its fully-relaxed geometry, computed in acetonitrile (isovalue: $0.002 \mathrm{e} \mathrm{bohr}^{-3}$ ). Such a triplet is basically a ${ }^{3} \mathrm{LC}$ state having an internal charge-transfer nature within the dmabpy ligand. 

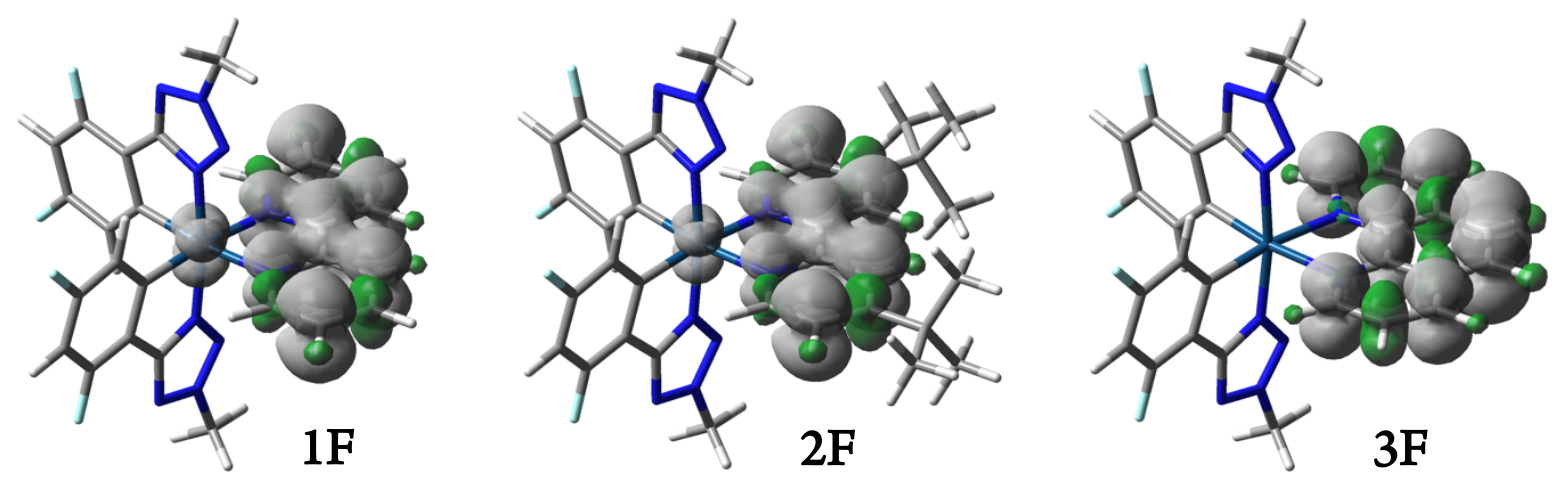

Figure S20. Spin-density distribution of the lowest triplet state of complexes 1F-3F in their fully-relaxed geometry, computed in acetonitrile (isovalues: $0.002 \mathrm{e} \mathrm{bohr}^{-3}$ ). For all the complexes, the lowest-energy triplet is a ${ }^{3} \mathrm{LC}$ state centered on the ancillary ligands, with some minor contribution from the iridium ion only in the case of the bpy-based complexes $1 \mathrm{~F}$ and $2 \mathrm{~F}$.

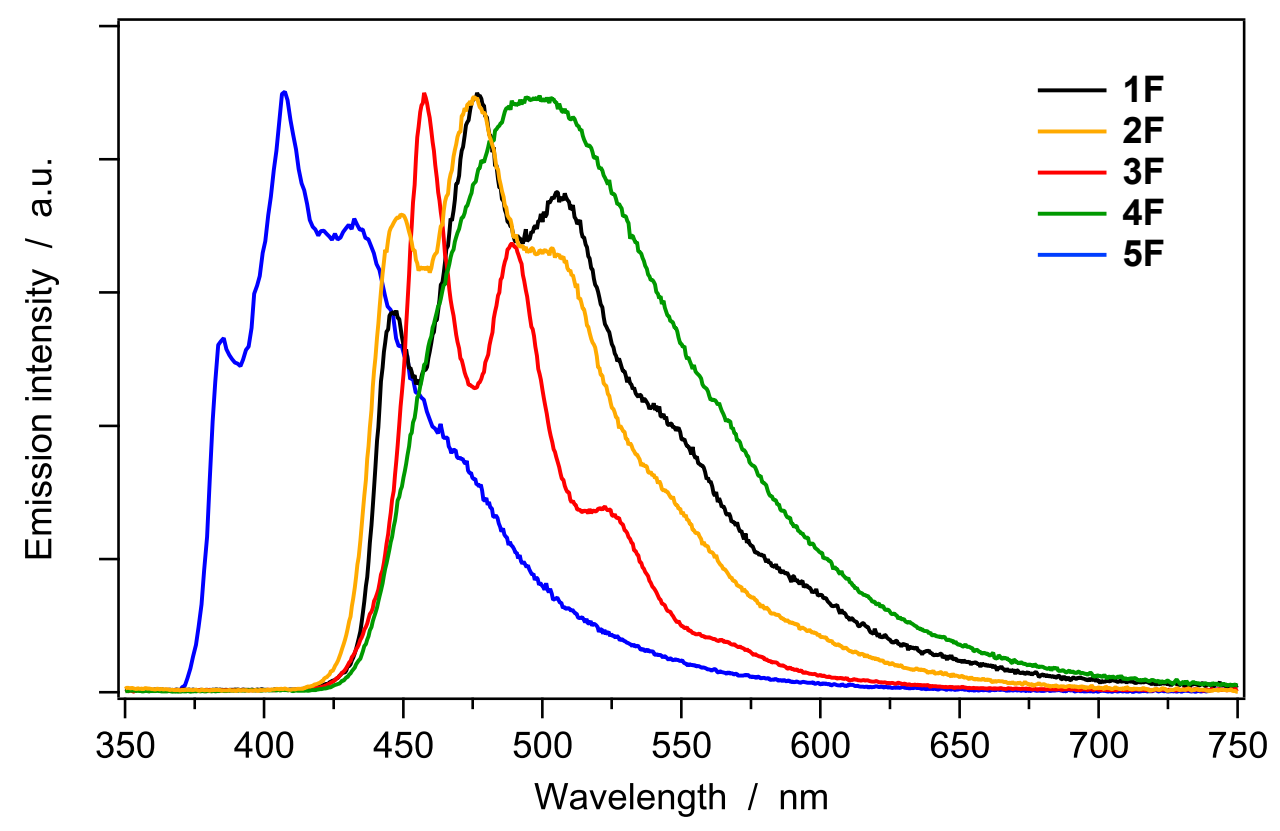

Figure S21. Normalized emission spectra of complexes 1F-5F in 1\% PMMA matrix at $298 \mathrm{~K}$. 


\section{DFT Optimized Geometries}

Table S4a. Fully-optimized ground-state geometry of complex 1F in acetonitrile (see Experimental Section for further details). The reported $x y z$ Cartesian coordinates are in Ångström.

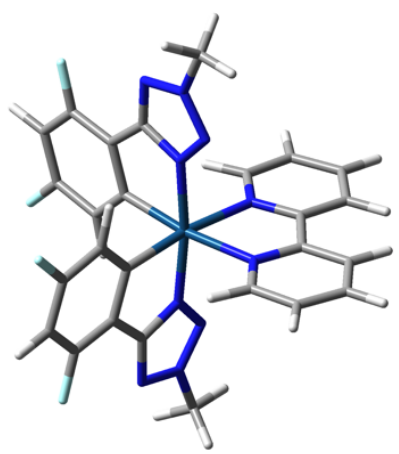

$\begin{array}{lrrr}\mathrm{C} & -3.180727 & -2.466354 & 2.407517 \\ \mathrm{C} & -2.509155 & -2.959479 & 1.305270 \\ \mathrm{C} & -1.539485 & -2.213203 & 0.642996 \\ \mathrm{C} & -1.203169 & -0.902493 & 1.079600 \\ \mathrm{C} & -1.880187 & -0.402588 & 2.193653 \\ \mathrm{C} & -2.840007 & -1.185526 & 2.823209 \\ \mathrm{H} & -3.935287 & -3.050608 & 2.922130 \\ \mathrm{H} & -1.682666 & 0.589988 & 2.594199 \\ \mathrm{C} & -0.785976 & -2.661142 & -0.500821 \\ \mathrm{~N} & 0.135121 & -1.773754 & -0.974782 \\ \mathrm{~N} & -0.767105 & -3.763010 & -1.236767 \\ \mathrm{~N} & 0.736978 & -2.305853 & -2.006585 \\ \mathrm{~N} & 0.176565 & -3.484580 & -2.132530 \\ \mathrm{C} & 0.562310 & -4.419715 & -3.174591 \\ \mathrm{H} & 0.930954 & -5.336293 & -2.711137 \\ \mathrm{H} & -0.307571 & -4.637632 & -3.796319 \\ \mathrm{H} & 1.346276 & -3.949057 & -3.766352 \\ \mathrm{I} r & 0.268789 & -0.000007 & 0.000006 \\ \mathrm{C} & -1.203118 & 0.902565 & -1.079585 \\ \mathrm{C} & -1.539342 & 2.213304 & -0.642997 \\ \mathrm{C} & -1.880176 & 0.402691 & -2.193626 \\ \mathrm{C} & -2.508962 & 2.959640 & -1.305280 \\ \mathrm{C} & -2.839941 & 1.185687 & -2.823193 \\ \mathrm{H} & -1.682728 & -0.589906 & -2.594157 \\ \mathrm{C} & -3.180568 & 2.466546 & -2.407521 \\ \mathrm{H} & -3.935087 & 3.050846 & -2.922142 \\ \mathrm{C} & -0.785802 & 2.661202 & 0.500818 \\ \mathrm{~N} & 0.135235 & 1.773755 & 0.974785 \\ \mathrm{~N} & -0.766858 & 3.763073 & 1.236756 \\ \mathrm{~N} & 0.737126 & 2.305820 & 2.006586 \\ \mathrm{~N} & 0.176792 & 3.484585 & 2.132523 \\ \mathrm{C} & 0.562602 & 4.419701 & 3.174576 \\ \mathrm{H} & 1.346538 & 3.948995 & 3.766338 \\ \mathrm{H} & -0.307262 & 4.637682 & 3.796305 \\ \mathrm{H} & 0.931307 & 5.336251 & 2.711114 \\ \mathrm{~N} & 1.966520 & -0.717628 & 1.120630 \\ \mathrm{C} & 3.183217 & -0.384440 & 0.629306 \\ \mathrm{C} & 1.879406 & -1.410760 & 2.262993 \\ \mathrm{C} & 3.183238 & 0.384235 & -0.629315 \\ \mathrm{C} & 4.346380 & -0.754125 & 1.298238 \\ \mathrm{C} & 2.999724 & -1.810805 & 2.974751\end{array}$

$\begin{array}{rrrr}\mathrm{H} & 0.873414 & -1.641881 & 2.606942 \\ \mathrm{C} & 4.346420 & 0.753837 & -1.298259 \\ \mathrm{~N} & 1.966560 & 0.717505 & -1.120629 \\ \mathrm{H} & 5.320184 & -0.480516 & 0.906619 \\ \mathrm{C} & 4.253458 & -1.474584 & 2.480908 \\ \mathrm{H} & 2.881921 & -2.371340 & 3.895908 \\ \mathrm{C} & 4.253538 & 1.474298 & -2.480930 \\ \mathrm{H} & 5.320209 & 0.480157 & -0.906649 \\ \mathrm{C} & 1.879484 & 1.410639 & -2.262994 \\ \mathrm{C} & 2.999823 & 1.810605 & -2.974762 \\ \mathrm{H} & 0.873504 & 1.641823 & -2.606938 \\ \mathrm{H} & 2.882050 & 2.371142 & -3.895922 \\ \mathrm{~F} & -3.472809 & 0.687042 & -3.886798 \\ \mathrm{~F} & -2.792499 & 4.185349 & -0.864854 \\ \mathrm{~F} & -2.792778 & -4.185162 & 0.864826 \\ \mathrm{~F} & -3.472837 & -0.686853 & 3.886823 \\ \mathrm{H} & 5.154613 & -1.767286 & 3.011196 \\ \mathrm{H} & 5.154708 & 1.766932 & -3.011229\end{array}$


Table S4b. Fully-optimized geometry of the ${ }^{3} \mathrm{LC}$ excited state of complex $1 \mathrm{~F}$ in acetonitrile (see Experimental Section for further details). The reported $x y z$ Cartesian coordinates are in Ångström.

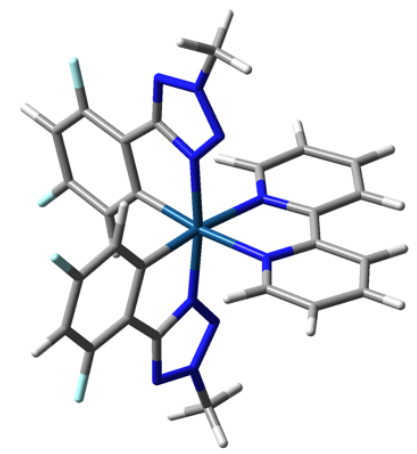

$\begin{array}{lrrr}\mathrm{C} & 3.185572 & -2.540723 & -2.339030 \\ \mathrm{C} & 2.510549 & -3.003728 & -1.225934 \\ \mathrm{C} & 1.540678 & -2.237629 & -0.586352 \\ \mathrm{C} & 1.210164 & -0.938031 & -1.057979 \\ \mathrm{C} & 1.891449 & -0.467964 & -2.181714 \\ \mathrm{C} & 2.849957 & -1.270113 & -2.788895 \\ \mathrm{H} & 3.939773 & -3.140122 & -2.836501 \\ \mathrm{H} & 1.698069 & 0.514502 & -2.608260 \\ \mathrm{C} & 0.782967 & -2.652129 & 0.567264 \\ \mathrm{~N} & -0.133451 & -1.748316 & 1.019803 \\ \mathrm{~N} & 0.753321 & -3.737584 & 1.326768 \\ \mathrm{~N} & -0.744357 & -2.254814 & 2.059271 \\ \mathrm{~N} & -0.191984 & -3.433341 & 2.212443 \\ \mathrm{C} & -0.585357 & -4.341685 & 3.275388 \\ \mathrm{H} & -0.954416 & -5.268033 & 2.832253 \\ \mathrm{H} & 0.281332 & -4.546807 & 3.905878 \\ \mathrm{H} & -1.370521 & -3.854292 & 3.851778 \\ \mathrm{Ir} & -0.262245 & 0.000250 & 0.000069 \\ \mathrm{C} & 1.211856 & 0.935741 & 1.058229 \\ \mathrm{C} & 1.545150 & 2.234529 & 0.586327 \\ \mathrm{C} & 1.891974 & 0.464533 & 2.182191 \\ \mathrm{C} & 2.516569 & 2.998720 & 1.225793 \\ \mathrm{C} & 2.852157 & 1.264791 & 2.789235 \\ \mathrm{H} & 1.696393 & -0.517399 & 2.608967 \\ \mathrm{C} & 3.190552 & 2.534553 & 2.339034 \\ \mathrm{H} & 3.945988 & 3.132476 & 2.836405 \\ \mathrm{C} & 0.788302 & 2.650466 & -0.567335 \\ \mathrm{~N} & -0.130006 & 1.748511 & -1.019738 \\ \mathrm{~N} & 0.760847 & 3.735926 & -1.326925 \\ \mathrm{~N} & -0.739954 & 2.256198 & -2.059185 \\ \mathrm{~N} & -0.185146 & 3.433563 & -2.212509 \\ \mathrm{C} & -0.576683 & 4.342607 & -3.275535 \\ \mathrm{H} & -1.362888 & 3.856786 & -3.851832 \\ \mathrm{H} & 0.290396 & 4.545861 & -3.906094 \\ \mathrm{H} & -0.943786 & 5.269772 & -2.832485 \\ \mathrm{~N} & -1.923165 & -0.773025 & -1.091232 \\ \mathrm{C} & -3.180213 & -0.393386 & -0.566250 \\ \mathrm{C} & -1.879131 & -1.560123 & -2.146642 \\ \mathrm{C} & -3.179514 & 0.399612 & 0.566019 \\ \mathrm{C} & -4.382363 & -0.857715 & -1.227587 \\ \mathrm{C} & -3.023874 & -2.043026 & -2.807125\end{array}$

$\begin{array}{rrrr}\mathrm{H} & -0.885384 & -1.825981 & -2.506011 \\ \mathrm{C} & -4.380850 & 0.866377 & 1.227120 \\ \mathrm{~N} & -1.921795 & 0.776759 & 1.091193 \\ \mathrm{H} & -5.351693 & -0.555452 & -0.846561 \\ \mathrm{C} & -4.295663 & -1.666707 & -2.321844 \\ \mathrm{H} & -2.908734 & -2.683564 & -3.674470 \\ \mathrm{C} & -4.292721 & 1.675192 & 2.321389 \\ \mathrm{H} & -5.350722 & 0.566088 & 0.845903 \\ \mathrm{C} & -1.876375 & 1.563774 & 2.146609 \\ \mathrm{C} & -3.020266 & 2.048953 & 2.806903 \\ \mathrm{H} & -0.882163 & 1.827683 & 2.506118 \\ \mathrm{H} & -2.903999 & 2.689287 & 3.674249 \\ \mathrm{~F} & 3.487485 & 0.795137 & 3.864042 \\ \mathrm{~F} & 2.798651 & 4.212102 & 0.752483 \\ \mathrm{~F} & 2.790085 & -4.217811 & -0.752897 \\ \mathrm{~F} & 3.486395 & -0.801518 & -3.863508 \\ \mathrm{H} & -5.192142 & 2.026935 & 2.817048 \\ \mathrm{H} & -5.195699 & -2.016620 & -2.817681\end{array}$


Table S4c. Fully-optimized geometry of the ${ }^{3}$ MLCT excited state of complex $1 \mathrm{~F}$ in acetonitrile (see Experimental Section for further details). The reported $x y z$ Cartesian coordinates are in Ångström.

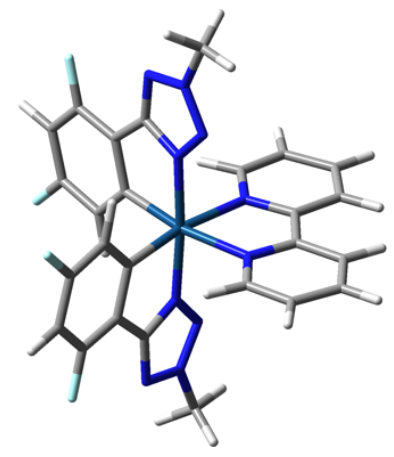

$\begin{array}{lrrr}\mathrm{C} & 2.978986 & -2.998764 & 2.112813 \\ \mathrm{C} & 3.271764 & -2.357654 & 0.917808 \\ \mathrm{C} & 2.385709 & -1.458201 & 0.346764 \\ \mathrm{C} & 1.130564 & -1.183470 & 0.973524 \\ \mathrm{C} & 0.839934 & -1.822926 & 2.185917 \\ \mathrm{C} & 1.759506 & -2.712033 & 2.720250 \\ \mathrm{H} & 3.677731 & -3.698556 & 2.558616 \\ \mathrm{H} & -0.087349 & -1.642758 & 2.723981 \\ \mathrm{C} & 2.606326 & -0.736721 & -0.882004 \\ \mathrm{~N} & 1.590378 & 0.083894 & -1.262582 \\ \mathrm{~N} & 3.594019 & -0.665647 & -1.760028 \\ \mathrm{~N} & 1.924982 & 0.681728 & -2.373880 \\ \mathrm{~N} & 3.118976 & 0.209996 & -2.639795 \\ \mathrm{C} & 3.870244 & 0.620074 & -3.815368 \\ \mathrm{H} & 4.803195 & 1.084165 & -3.492251 \\ \mathrm{H} & 4.078270 & -0.260375 & -4.424967 \\ \mathrm{H} & 3.257106 & 1.332559 & -4.365060 \\ \mathrm{Ir} & -0.000001 & 0.169299 & -0.000000 \\ \mathrm{C} & -1.130601 & -1.183432 & -0.973532 \\ \mathrm{C} & -2.385763 & -1.458115 & -0.346784 \\ \mathrm{C} & -0.839982 & -1.822901 & -2.185920 \\ \mathrm{C} & -3.271847 & -2.357533 & -0.917839 \\ \mathrm{C} & -1.759581 & -2.711976 & -2.720262 \\ \mathrm{H} & 0.087315 & -1.642772 & -2.723973 \\ \mathrm{C} & -2.979079 & -2.998659 & -2.112839 \\ \mathrm{H} & -3.677845 & -3.698426 & -2.558647 \\ \mathrm{C} & -2.606364 & -0.736631 & 0.881985 \\ \mathrm{~N} & -1.590388 & 0.083942 & 1.262576 \\ \mathrm{~N} & -3.594068 & -0.665514 & 1.759993 \\ \mathrm{~N} & -1.924995 & 0.681811 & 2.373855 \\ \mathrm{~N} & -3.118969 & 0.210050 & 2.639809 \\ \mathrm{C} & -3.870192 & 0.620078 & 3.815428 \\ \mathrm{H} & -3.257034 & 1.332540 & 4.365126 \\ \mathrm{H} & -4.078200 & -0.260395 & 4.425000 \\ \mathrm{H} & -4.803152 & 1.084182 & 3.492360 \\ \mathrm{~N} & 0.922859 & 1.844787 & 0.957833 \\ \mathrm{C} & 0.485076 & 3.086502 & 0.516129 \\ \mathrm{C} & 1.855448 & 1.777701 & 1.934606 \\ \mathrm{C} & -0.484965 & 3.086523 & -0.516107 \\ \mathrm{C} & 1.024526 & 4.254910 & 1.112928 \\ \mathrm{C} & 2.403241 & 2.885878 & 2.531330\end{array}$

$\begin{array}{rrrr}\mathrm{H} & 2.154802 & 0.777489 & 2.241938 \\ \mathrm{C} & -1.024367 & 4.254955 & -1.112902 \\ \mathrm{~N} & -0.922798 & 1.844828 & -0.957815 \\ \mathrm{H} & 0.679207 & 5.229435 & 0.780345 \\ \mathrm{C} & 1.966121 & 4.162988 & 2.101079 \\ \mathrm{H} & 3.143608 & 2.767123 & 3.314991 \\ \mathrm{C} & -1.965969 & 4.163074 & -2.101051 \\ \mathrm{H} & -0.679008 & 5.229465 & -0.780317 \\ \mathrm{C} & -1.855393 & 1.777782 & -1.934585 \\ \mathrm{C} & -2.403143 & 2.885983 & -2.531304 \\ \mathrm{H} & -2.154788 & 0.777583 & -2.241918 \\ \mathrm{H} & -3.143517 & 2.767260 & -3.314963 \\ \mathrm{~F} & -1.477338 & -3.322090 & -3.864745 \\ \mathrm{~F} & -4.426433 & -2.607600 & -0.315194 \\ \mathrm{~F} & 4.426335 & -2.607762 & 0.315152 \\ \mathrm{~F} & 1.477254 & -3.322133 & 3.864739 \\ \mathrm{H} & 2.371496 & 5.063946 & 2.553442 \\ \mathrm{H} & -2.371308 & 5.064050 & -2.553410\end{array}$


Table S5a. Fully-optimized ground-state geometry of complex 2F in acetonitrile (see Experimental Section for further details). The reported $x y z$ Cartesian coordinates are in Ångström.

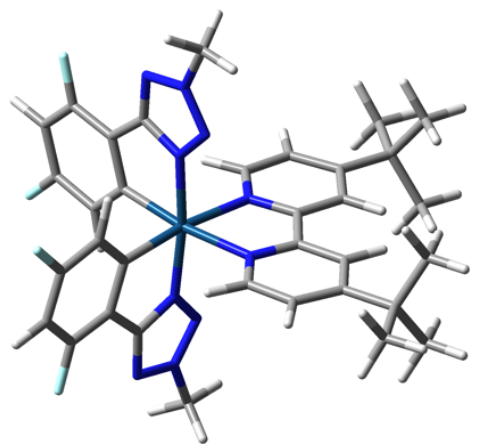

\begin{tabular}{|c|c|c|}
\hline 4.095879 & -3.447555 & 0.143084 \\
\hline 3.421600 & -2.985722 & 1.257129 \\
\hline 2.452404 & -1.991392 & 1.167944 \\
\hline 2.119638 & -1.405856 & -0.084518 \\
\hline 2.800183 & -1.871675 & -1.211417 \\
\hline 3.758498 & -2.868050 & -1.073449 \\
\hline 4.849815 & -4.223449 & 0.215274 \\
\hline 2.606717 & -1.480330 & -2.208518 \\
\hline 1.695170 & -1.470127 & 2.277675 \\
\hline 0.777679 & -0.512509 & 1.957987 \\
\hline 1.669005 & -1.698296 & 3.582918 \\
\hline 0.170468 & -0.132630 & 3.052096 \\
\hline 0.725103 & -0.855991 & 3.994954 \\
\hline 0.329993 & -0.749093 & 5.388223 \\
\hline-0.062031 & -1.711213 & 5.722358 \\
\hline 1.201074 & -0.472138 & 5.984112 \\
\hline-0.438422 & 0.019965 & 5.454976 \\
\hline 0.648368 & -0.000013 & 0.000030 \\
\hline 2.119570 & 1.405917 & 0.084331 \\
\hline 2.452062 & 1.991495 & -1.168186 \\
\hline 2.800299 & 1.871765 & 1.211107 \\
\hline 3.421175 & 2.985890 & -1.257538 \\
\hline 3.758521 & 2.868206 & 1.072973 \\
\hline 2.607051 & 1.480394 & 2.208240 \\
\hline 4.095633 & 3.447752 & -0.143613 \\
\hline 4.849504 & 4.223698 & -0.215935 \\
\hline 1.694649 & 1.470206 & -2.277784 \\
\hline 0.777294 & 0.512507 & -1.957950 \\
\hline 1.668199 & 1.698429 & -3.583012 \\
\hline 0.169892 & 0.132625 & -3.051951 \\
\hline 0.724260 & 0.856087 & -3.994887 \\
\hline 0.328846 & 0.749246 & -5.388075 \\
\hline-0.439529 & -0.019864 & -5.454702 \\
\hline 1.199812 & 0.472390 & -5.984176 \\
\hline-0.063323 & 1.711358 & -5.722063 \\
\hline-1.048315 & -1.305425 & -0.241851 \\
\hline-2.268412 & -0.723583 & -0.146709 \\
\hline-0.976850 & -2.612642 & -0.511279 \\
\hline-2.268426 & 0.723468 & 0.146952 \\
\hline-3.426750 & -1.465682 & -0.324566 \\
\hline-2.100531 & -3.402564 & -0.696247 \\
\hline 0.024258 & -3.033350 & -0.582636 \\
\hline-3.426778 & 1.465546 & 0.324807 \\
\hline-1.048341 & 1.305327 & 0.24212 \\
\hline-4.39183 & -0.973452 & -0.24783 \\
\hline-3.370987 & -2.834729 & -0.605230 \\
\hline
\end{tabular}

$-4.456433$

2.834584

0.973308

2.612539

3.402439

3. 033258

4.456306

3.298309

3.508546

$-3.508341$

$-3.298127$

$-3.626951$

3. 626781

3. 546310

2. 516107

4.119313

3.968350

3. 022158

3. 059416

3.593601

1. 979366

5.095693

5.217948

5.591644

5.621867

$-3.022320$

$-3.593797$

$-1.979544$

$-3.059523$

$-5.095844$

$-5.218045$

$-5.591829$

$-5.622023$

$-3.546555$

$-3.968554$

$-2.516379$

$-4.119636$
$-0.912046$

0.605516

0.248035

0.511584

0.696556

0.582964

0.912380

2. 164807

$-2.451480$

2. 451027

$-2.165395$

$-0.793870$

0.794124

$-0.495469$

$-0.747276$

$-0.373919$

$-1.343528$

1.958041

2.890041

2.106437

1. 772495

1.104779

2. 032551

0.290771

1.233674

$-1.957774$

$-2.106202$

$-1.772200$

$-2.889765$

$-1.104569$

$-2.032292$

$-0.290532$

$-1.233582$

0.495722

1. 343784

0.747533

0.374164 
Table S5b. Fully-optimized geometry of the ${ }^{3} \mathrm{LC}$ excited state of complex $2 \mathrm{~F}$ in acetonitrile (see Experimental Section for further details). The reported $x y z$ Cartesian coordinates are in Ångström.

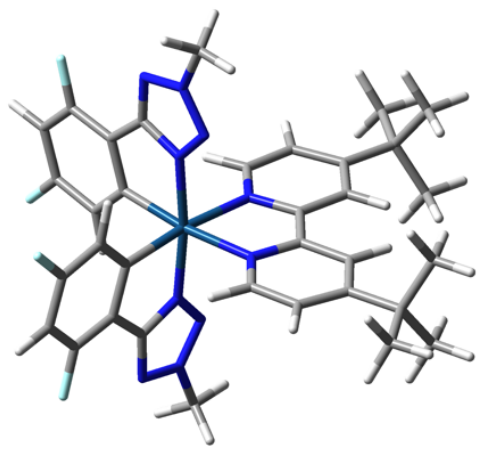

\begin{tabular}{|c|c|c|c|}
\hline $\mathrm{C}$ & -4.105343 & -3.456797 & 0.067309 \\
\hline $\mathrm{C}$ & -3.429949 & -3.065360 & -1.072762 \\
\hline & -2.461992 & -2.065999 & -1.044298 \\
\hline & -2.132213 & -1.404292 & 0.170005 \\
\hline & -2.814096 & -1.797877 & 1.322730 \\
\hline & -3.771487 & -2.801743 & 1.24593 \\
\hline & -4.858236 & -4.236642 & 0.04263 \\
\hline & -2.621705 & -1.342941 & 2.292644 \\
\hline & -1.705348 & -1.610415 & -2.18314 \\
\hline & -0.791705 & -0.6309 & -1.92345 \\
\hline & -1.675808 & -1.920207 & -3.47 \\
\hline & -0.183158 & -0.3193 & -3.0 \\
\hline & -0.733685 & -1.1023 & -3.9 \\
\hline & -0.338501 & -1.08044 & -5.331 \\
\hline & 0.045638 & -2.06377 & -5.6 \\
\hline & -1.207768 & -0.8323 & -5.942 \\
\hline & 0.435943 & -0.3227 & -5.443 \\
\hline & -0.662239 & -0.000 & 0.0 \\
\hline & -2.13221 & 1.404 & $-0.17 c$ \\
\hline & -2.461994 & 2.0659 & 1.044 \\
\hline & -2.81409 & 1.7978 & -1.322 \\
\hline & -3.429956 & 3.065 & 1.072 \\
\hline & -3.771494 & 2.8017 & -1.2459 \\
\hline & -2.62 & 1.342 & -2.2 \\
\hline & -4.105 & $3.456^{\circ}$ & -0.06 \\
\hline & -4.858249 & 4.2366 & -0.04 \\
\hline & -1.70 & 1.610 & 2.1 \\
\hline & -0.79 & 0.63 & 1.92 \\
\hline & -1.675 & 1.920 & 3.4 \\
\hline & -0.18 & 0.31 & \\
\hline & -0.73 & 1.10 & \\
\hline & -0.338 & 1.0804 & 5.33 \\
\hline & 0.43 & 0.32 & \\
\hline & -1.20774 & 0.8324 & 5.94 \\
\hline & 0.045656 & 2.0637 & 5.60757 \\
\hline & 1.004541 & -1.29938 & 0 . \\
\hline & 2.26128 & -0.6715 & 0.16226 \\
\hline & 0.975885 & -2.5789 & 0.59702 \\
\hline & 2.261286 & 0.6715 & -0.1 \\
\hline & 3.462805 & -1.457897 & 0.355930 \\
\hline & 2.127228 & -3.3735 & 0.78537 \\
\hline & -0.01228 & -3.027864 & \\
\hline & 3.462804 & 1.457898 & -0.355929 \\
\hline & 1.00 & 1.29938 & -0.3 \\
\hline & 4.423 & -0.962 & \\
\hline & 3.4118 & -2.797240 & 0.664 \\
\hline
\end{tabular}

$\begin{array}{rrrr}\mathrm{H} & 1.988199 & -4.421578 & 1.025718 \\ \mathrm{C} & 3.411835 & 2.797240 & -0.664448 \\ \mathrm{H} & 4.423544 & 0.962020 & -0.254931 \\ \mathrm{C} & 0.975883 & 2.578918 & -0.597024 \\ \mathrm{C} & 2.127225 & 3.373512 & -0.785381 \\ \mathrm{H} & -0.012289 & 3.027861 & -0.697261 \\ \mathrm{H} & 1.988195 & 4.421575 & -1.025727 \\ \mathrm{~F} & -4.408862 & 3.162921 & -2.361156 \\ \mathrm{~F} & -3.708093 & 3.662859 & 2.231479 \\ \mathrm{~F} & -3.708083 & -3.662866 & -2.231479 \\ \mathrm{~F} & -4.408853 & -3.162935 & 2.361157 \\ \mathrm{C} & 4.695057 & -3.597080 & 0.860275 \\ \mathrm{C} & 4.695054 & 3.597080 & -0.860281 \\ \mathrm{C} & 5.512112 & 3.556869 & 0.436378 \\ \mathrm{H} & 5.794982 & 2.534879 & 0.713910 \\ \mathrm{H} & 6.435282 & 4.136234 & 0.311113 \\ \mathrm{H} & 4.947255 & 3.991915 & 1.269978 \\ \mathrm{C} & 5.506329 & 2.975644 & -2.002980 \\ \mathrm{H} & 4.936566 & 2.985998 & -2.940203 \\ \mathrm{H} & 6.427672 & 3.550677 & -2.157898 \\ \mathrm{H} & 5.793187 & 1.939295 & -1.790680 \\ \mathrm{C} & 4.409756 & 5.056400 & -1.207767 \\ \mathrm{H} & 3.844557 & 5.152285 & -2.143289 \\ \mathrm{H} & 3.855348 & 5.566847 & -0.410410 \\ \mathrm{H} & 5.358967 & 5.588041 & -1.340662 \\ \mathrm{C} & 5.506334 & -2.975646 & 2.002974 \\ \mathrm{H} & 6.427677 & -3.550679 & 2.157889 \\ \mathrm{H} & 5.793191 & -1.939296 & 1.790676 \\ \mathrm{H} & 4.936572 & -2.986002 & 2.940198 \\ \mathrm{C} & 4.409760 & -5.056400 & 1.207758 \\ \mathrm{H} & 3.844562 & -5.152288 & 2.143281 \\ \mathrm{H} & 3.855352 & -5.566846 & 0.410401 \\ \mathrm{H} & 5.358972 & -5.588042 & 1.340651 \\ \mathrm{C} & 5.512114 & -3.556865 & -0.436385 \\ \mathrm{H} & 4.947255 & -3.991910 & -1.269985 \\ \mathrm{H} & 5.794982 & -2.534875 & -0.713915 \\ \mathrm{H} & 6.435284 & -4.136230 & -0.311123\end{array}$


Table S6a. Fully-optimized ground-state geometry of complex 3F in acetonitrile (see Experimental Section for further details). The reported $x y z$ Cartesian coordinates are in Ångström.

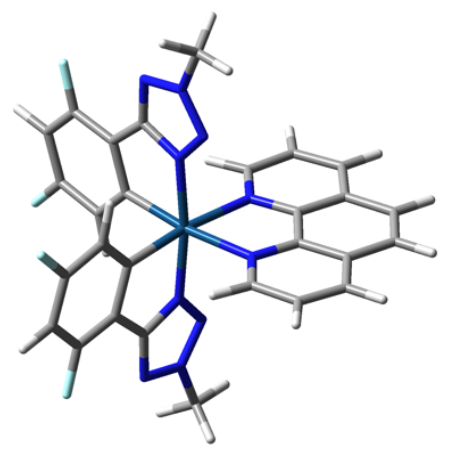

$\begin{array}{lrrr}\text { C } & 3.358973 & -2.554551 & -2.352107 \\ \mathrm{C} & 2.692781 & -3.011324 & -1.231080 \\ \mathrm{C} & 1.731080 & -2.240738 & -0.584958 \\ \mathrm{C} & 1.397743 & -0.942364 & -1.058958 \\ \mathrm{C} & 2.069964 & -0.478761 & -2.191296 \\ \mathrm{C} & 3.021704 & -1.284961 & -2.803448 \\ \mathrm{H} & 4.107271 & -3.157569 & -2.854073 \\ \mathrm{H} & 1.875567 & 0.502497 & -2.620075 \\ \mathrm{C} & 0.982713 & -2.649457 & 0.576905 \\ \mathrm{~N} & 0.070501 & -1.742381 & 1.030357 \\ \mathrm{~N} & 0.961299 & -3.729128 & 1.344824 \\ \mathrm{~N} & -0.529069 & -2.240378 & 2.080275 \\ \mathrm{~N} & 0.024669 & -3.418499 & 2.237522 \\ \mathrm{C} & -0.360635 & -4.319865 & 3.309000 \\ \mathrm{H} & -0.741404 & -5.245993 & 2.875265 \\ \mathrm{H} & 0.512489 & -4.527908 & 3.929574 \\ \mathrm{H} & -1.135286 & -3.825176 & 3.893404 \\ \mathrm{I} r & -0.061701 & 0.00000 & -0.000006 \\ \mathrm{C} & 1.397741 & 0.942371 & 1.058943 \\ \mathrm{C} & 1.731062 & 2.240752 & 0.584949 \\ \mathrm{C} & 2.069979 & 0.478768 & 2.191270 \\ \mathrm{C} & 2.692761 & 3.011343 & 1.231066 \\ \mathrm{C} & 3.021717 & 1.284973 & 2.803419 \\ \mathrm{H} & 1.875599 & -0.502495 & 2.620047 \\ \mathrm{C} & 3.358969 & 2.554569 & 2.352084 \\ \mathrm{H} & 4.107267 & 3.157591 & 2.854046 \\ \mathrm{C} & 0.982677 & 2.649474 & -0.576902 \\ \mathrm{~N} & 0.070478 & 1.742390 & -1.030363 \\ \mathrm{~N} & 0.961230 & 3.729163 & -1.344794 \\ \mathrm{~N} & -0.529118 & 2.240400 & -2.080260 \\ \mathrm{~N} & 0.024593 & 3.418537 & -2.237487 \\ \mathrm{C} & -0.360735 & 4.319914 & -3.308945 \\ \mathrm{H} & -1.135425 & 3.825247 & -3.893316 \\ \mathrm{H} & 0.512366 & 4.527936 & -3.929559 \\ \mathrm{H} & -0.741460 & 5.246051 & -2.875191 \\ \mathrm{~N} & -1.754229 & -0.782369 & -1.100861 \\ \mathrm{C} & -2.957075 & -0.413618 & -0.583755 \\ \mathrm{C} & -1.726940 & -1.544326 & -2.187748 \\ \mathrm{C} & -2.957076 & 0.413579 & 0.583764 \\ \mathrm{C} & -4.178417 & -0.815531 & -1.156730 \\ \mathrm{C} & -2.894866 & -1.987260 & -2.822048 \\ \mathrm{H} & -0.742244 & -1.809967 & -2.567867 \\ \mathrm{C} & -4.178419 & 0.815467 & 1.156755 \\ \mathrm{~N} & -1.754231 & 0.782356 & 1.100855 \\ \mathrm{C} & -4.119285 & -1.625188 & -2.307351 \\ \mathrm{H} & -2.815326 & -2.608736 & -3.707583\end{array}$

$\begin{array}{rrrr}\mathrm{C} & -4.119291 & 1.625128 & 2.307373 \\ \mathrm{C} & -1.726945 & 1.544316 & 2.187740 \\ \mathrm{C} & -2.894873 & 1.987227 & 2.822053 \\ \mathrm{H} & -0.742250 & 1.809978 & 2.567847 \\ \mathrm{H} & -2.815335 & 2.608708 & 3.707585 \\ \mathrm{~F} & 3.649906 & 0.821213 & 3.885413 \\ \mathrm{~F} & 2.973662 & 4.224726 & 0.756127 \\ \mathrm{~F} & 2.973697 & -4.224701 & -0.756135 \\ \mathrm{~F} & 3.649878 & -0.821203 & -3.885451 \\ \mathrm{H} & -5.043140 & -1.954840 & -2.776745 \\ \mathrm{H} & -5.043146 & 1.954761 & 2.776777 \\ \mathrm{C} & -5.405397 & 0.391329 & 0.555602 \\ \mathrm{C} & -5.405396 & -0.391423 & -0.555560 \\ \mathrm{H} & -6.339415 & -0.711794 & -1.010697 \\ \mathrm{H} & -6.339418 & 0.711676 & 1.010754\end{array}$


Table S6b. Fully-optimized geometry of the ${ }^{3} \mathrm{LC}$ excited state of complex $3 \mathrm{~F}$ in acetonitrile (see Experimental Section for further details). The reported $x y z$ Cartesian coordinates are in Ångström.

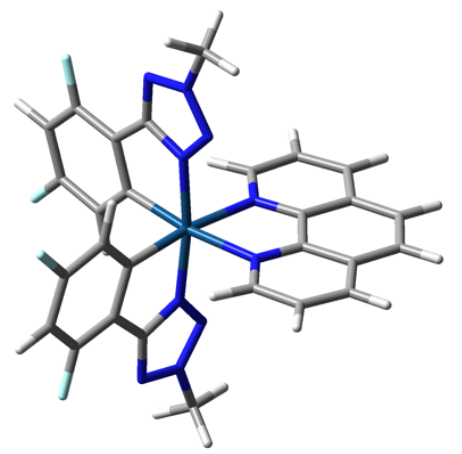

\begin{tabular}{|c|c|c|c|}
\hline $\mathrm{C}$ & -3.358487 & 2.523126 & -2.370584 \\
\hline $\mathrm{C}$ & -2.695092 & 2.989840 & -1.252052 \\
\hline & -1.730321 & 2.227634 & -0.600631 \\
\hline & -1.391275 & 0.927912 & -1.066359 \\
\hline & -2.060008 & 0.454211 & -2.196299 \\
\hline & -3.015043 & 1.252489 & -2.814064 \\
\hline & -4.109203 & 3.119624 & -2.876700 \\
\hline & -1.860673 & -0.528496 & -2.619468 \\
\hline & -0.983988 & 2.648044 & \\
\hline & -0.064843 & 1.750020 & 1.01503 \\
\hline & -0.970063 & 3.730942 & 1.3216 \\
\hline & 0.531018 & 2.256065 & \\
\hline & -0.030969 & 3.430717 & .21 \\
\hline & 0.348507 & 4.339725 & 3.2826 \\
\hline & 0.723407 & 5.2660 & \\
\hline & -0.526178 & 4.545116 & 3.9 \\
\hline & 1.126141 & 3.852755 & 3.8 \\
\hline$r$ & 0.07 & 0.00 & \\
\hline & -1.39127 & -0.927913 & 1.0 \\
\hline & -1.730319 & -2.227635 & 0.600 \\
\hline & -2.060008 & -0.4542 & 2.1 \\
\hline & -2.695090 & -2.989 & 1.2 \\
\hline & -3.015043 & -1.252492 & 2.81 \\
\hline & -1.86 & 0.528 & 2.6 \\
\hline & -3.358486 & -2.523129 & $2.3^{\circ}$ \\
\hline & -4.10 & -3.11 & 2.87 \\
\hline & -0.98 & -2.64 & -0.5 \\
\hline & -0.06 & -1.750 & -1.0 \\
\hline & -0.970060 & -3.730 & $-1 \cdot 3$ \\
\hline & 0.53101 & -2.256063 & -2.0 \\
\hline & -0.03096 & -3.4307 & -2.21 \\
\hline & 0.348513 & -4.339725 & -3.2826 \\
\hline & 1.126168 & -3.852765 & -3 \\
\hline & -0.526164 & -4.545095 & -3.9018 \\
\hline & 0.723388 & -5.266064 & -2.84428 \\
\hline & 1.771658 & 0.746644 & -1.1 \\
\hline & 2.95448 & 0.404852 & -0.61054 \\
\hline & 1.708760 & 1.486667 & -2.25140 \\
\hline & 2.9544 & -0.404849 & 0.6 \\
\hline & 4.191932 & 0.790651 & -1.214829 \\
\hline & 2.86024 & 1.902707 & -2.91 \\
\hline & 0.71 & 1.723637 & -2.611 \\
\hline & 4.191932 & -0.790648 & 1.21482 \\
\hline & & -0.746642 & 1.1 \\
\hline & & 1.566622 & -2 . \\
\hline & 2.767173 & 2.490316 & -3.8 \\
\hline
\end{tabular}

$\begin{array}{lrrr}\mathrm{C} & 4.103523 & -1.566619 & 2.407612 \\ \mathrm{C} & 1.708761 & -1.486665 & 2.251401 \\ \mathrm{C} & 2.860243 & -1.902705 & 2.910526 \\ \mathrm{H} & 0.711656 & -1.723636 & 2.611947 \\ \mathrm{H} & 2.767174 & -2.490313 & 3.818327 \\ \mathrm{~F} & -3.640092 & -0.779219 & 3.893468 \\ \mathrm{~F} & -2.981484 & -4.204590 & 0.784572 \\ \mathrm{~F} & -2.981488 & 4.204588 & -0.784573 \\ \mathrm{~F} & -3.640092 & 0.779216 & -3.893468 \\ \mathrm{H} & 5.016777 & 1.881243 & -2.905840 \\ \mathrm{H} & 5.016778 & -1.881240 & 2.905841 \\ \mathrm{C} & 5.381714 & -0.401022 & 0.617939 \\ \mathrm{C} & 5.381714 & 0.401025 & -0.617938 \\ \mathrm{H} & 6.330538 & 0.690508 & -1.061487 \\ \mathrm{H} & 6.330538 & -0.690505 & 1.061487\end{array}$


Table S6c. Fully-optimized geometry of the ${ }^{3} \mathrm{MLCT}$ excited state of complex $3 \mathrm{~F}$ in acetonitrile (see Experimental Section for further details). The reported $x y z$ Cartesian coordinates are in Ångström.

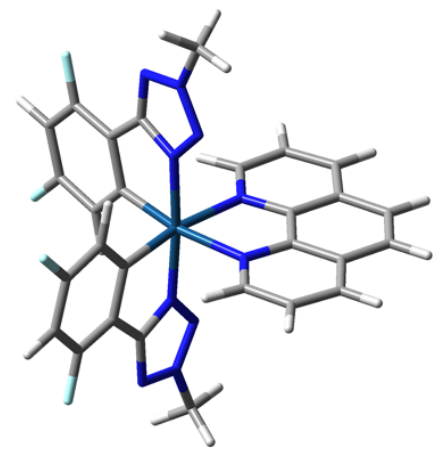

\begin{tabular}{|c|c|c|c|}
\hline $\mathrm{C}$ & 3.157501 & -3.050452 & -2.078482 \\
\hline $\mathrm{C}$ & 2.529045 & -3.310925 & -0.869204 \\
\hline$C$ & 1.643834 & -2.405102 & -0.307553 \\
\hline C & 1.372319 & -1.161899 & -0.959104 \\
\hline & 1.996887 & -0.904604 & -2.186842 \\
\hline & 2.872055 & -1.843373 & -2.710756 \\
\hline & 3.846185 & -3.764901 & -2.51664 \\
\hline & 1.816148 & 0.011093 & -2.744034 \\
\hline & 0.931922 & -2.593303 & \\
\hline & 0.123605 & -1.562357 & 1.29796 \\
\hline & 0.857108 & -3.563130 & 1.829 \\
\hline & -0.471096 & -1.870 & \\
\hline & -0.008936 & -3.063086 & 2.7 \\
\hline & -0.419176 & -3.787843 & 3.897 \\
\hline & -0.892860 & -4.722 & \\
\hline & 0.462602 & -3.9915 & 4.5 \\
\hline & -1.123632 & -3.15814 & 4.4 \\
\hline Ir & 0.04 & -0.00 & \\
\hline & 1.372302 & 1.16 & \\
\hline & 1.643814 & 2.4051 & 0.3 \\
\hline & 1.996 & 0.904 & 2.1 \\
\hline & 2.525 & 3.31 & 0.8 \\
\hline & 2.872022 & 1.843 & 2.71 \\
\hline & 1.81 & -0.01 & 2.7 \\
\hline & 3.15 & 3 & 2.0 \\
\hline & 3.84 & 3.764 & 2.51 \\
\hline & 0.93 & 2.593 & -0.9 \\
\hline & 0.12 & 1.562 & -1.2 \\
\hline & 0.85 & 3.563 & -1.82 \\
\hline & -0.47 & 1.870 & -2.4 \\
\hline & -0.00 & 3.06 & -2.70 \\
\hline & -0.419214 & 3.7878 & -3.897 \\
\hline & $-1 \cdot 12$ & 3.158 & -4 . \\
\hline & 0.462 & 3.9914 & -4.5061 \\
\hline & -0.892891 & 4.7228 & -3.59437 \\
\hline & -1.63 & -0.959 & -0.9 \\
\hline & -2.85812 & -0.49606 & -0.48962 \\
\hline & -1.620015 & -1.921632 & -1.88928 \\
\hline & -2.85 & 0.49 & 0 \\
\hline & -4.080293 & -1.017888 & -1.005118 \\
\hline & -2.769821 & -2.462651 & -2.42621 \\
\hline & -0.639647 & $-2.2557 c$ & -2.22 \\
\hline & -4.080304 & 1.017862 & $1.00510^{\circ}$ \\
\hline & -1.631777 & 0.9591 & \\
\hline & -4.024 & -2.0051 & -1.97 \\
\hline & -2.688403 & -3.230265 & $-3.18 \varepsilon$ \\
\hline
\end{tabular}

$\begin{array}{lrrr}\mathrm{C} & -4.024070 & 2.005103 & 1.977277 \\ \mathrm{C} & -1.620036 & 1.921619 & 1.889291 \\ \mathrm{C} & -2.769848 & 2.462630 & 2.426213 \\ \mathrm{H} & -0.639672 & 2.255698 & 2.224839 \\ \mathrm{H} & -2.688438 & 3.230244 & 3.188705 \\ \mathrm{~F} & 3.469090 & 1.592901 & 3.869411 \\ \mathrm{~F} & 2.777341 & 4.453678 & 0.243766 \\ \mathrm{~F} & 2.777367 & -4.453664 & -0.243788 \\ \mathrm{~F} & 3.469131 & -1.592841 & -3.869394 \\ \mathrm{H} & -4.946302 & -2.415148 & -2.383174 \\ \mathrm{H} & -4.946328 & 2.415116 & 2.383160 \\ \mathrm{C} & -5.304942 & 0.482608 & 0.476855 \\ \mathrm{C} & -5.304937 & -0.482638 & -0.476875 \\ \mathrm{H} & -6.242293 & -0.876165 & -0.865738 \\ \mathrm{H} & -6.242303 & 0.876132 & 0.865711\end{array}$


Table S7a. Fully-optimized ground-state geometry of complex 4F in acetonitrile (see Experimental Section for further details). The reported $x y z$ Cartesian coordinates are in Ångström.

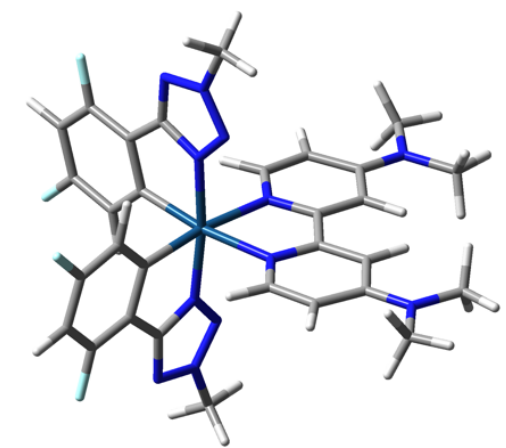

\begin{tabular}{|c|c|c|c|}
\hline C & 3.881378 & 3.460576 & -0.027292 \\
\hline & 3.203513 & 3.038807 & -1.155010 \\
\hline & 2.238193 & 2.038273 & -1.099562 \\
\hline $\mathrm{C}$ & 1.911342 & 1.404267 & 0.131156 \\
\hline & 2.597231 & 1.829768 & 1.271636 \\
\hline & 3.551599 & 2.833285 & 1.167832 \\
\hline & 4.632329 & 4.241355 & -0.073219 \\
\hline & 2.408909 & 1.398712 & 2.253429 \\
\hline & 1.479974 & 1.553538 & -2.22516 \\
\hline & 0.567938 & 0.579663 & -1.93826 \\
\hline & 1.450654 & 1.828794 & -3.52 \\
\hline & -0.038521 & 0.236614 & -3.0 \\
\hline & 0.510787 & 0.996770 & -3.96 \\
\hline & 0.112830 & 0.939073 & -5.35 \\
\hline & -0.291159 & 1.908570 & -5.65 \\
\hline & 0.984756 & 0.694790 & -5.966208 \\
\hline & -0.647320 & 0.164654 & -5.4 \\
\hline Ir & 0.442223 & -0.000 & -0.0 \\
\hline & 1.911340 & -1.404269 & -0.13 \\
\hline C & 2.238186 & -2.038277 & 1.09 \\
\hline & 2.597232 & -1.829772 & $-1 \cdot 2$ \\
\hline & 3.203503 & -3.038814 & 1.15 \\
\hline & 3.551597 & -2.833291 & -1.16 \\
\hline & 2.408 & -1.398715 & -2.2 \\
\hline & 3.881370 & -3.460584 & 0 \\
\hline & 4.632320 & -4.241364 & 0.07 \\
\hline & 1.479 & -1.553 & 2.2 \\
\hline & 0.567932 & -0.5796 & 1.9 \\
\hline & 1.450640 & -1.8287 & 3.52 \\
\hline & -0.038541 & -0.236624 & \\
\hline & 0.510785 & -0.996761 & 3. \\
\hline & 0.112841 & -0.939046 & 5.3573 \\
\hline & -0.647309 & -0.164625 & \\
\hline & 0.984772 & -0.694756 & 5.9662 \\
\hline & -0.291145 & -1.908539 & 5.65407 \\
\hline & -1.254382 & 1.290203 & 0 . \\
\hline & -2.480201 & 0.718501 & 0.18006 \\
\hline & -1.191180 & 2.591738 & 0.6128 \\
\hline & -2.4802 & -0.718499 & -0.18 \\
\hline & -3.640474 & 1.434528 & 0.40333 \\
\hline & -2.296137 & 3.37588 & \\
\hline & -0.190546 & 3.0125 & \\
\hline & -3.640475 & -1.434526 & -0.40333 \\
\hline & $-1 \cdot 2$ & -1.25 & -0 \\
\hline & -4 . & 0.93 & \\
\hline
\end{tabular}

$\begin{array}{lrrr}\mathrm{C} & -3.586399 & 2.806139 & 0.741615 \\ \mathrm{H} & -2.151466 & 4.418072 & 1.103263 \\ \mathrm{C} & -3.586401 & -2.806137 & -0.741616 \\ \mathrm{H} & -4.598976 & -0.935941 & -0.325460 \\ \mathrm{C} & -1.191182 & -2.591736 & -0.612865 \\ \mathrm{C} & -2.296140 & -3.375879 & -0.843275 \\ \mathrm{H} & -0.190549 & -3.012537 & -0.693807 \\ \mathrm{H} & -2.151469 & -4.418069 & -1.103270 \\ \mathrm{~N} & -4.708151 & 3.530715 & 0.958189 \\ \mathrm{~N} & -4.708154 & -3.530712 & -0.958188 \\ \mathrm{C} & -6.014612 & -2.904846 & -0.853048 \\ \mathrm{H} & -6.133127 & -2.092740 & -1.581964 \\ \mathrm{H} & -6.785117 & -3.650272 & -1.051203 \\ \mathrm{H} & -6.186597 & -2.499631 & 0.152327 \\ \mathrm{C} & -4.604955 & -4.935873 & -1.312609 \\ \mathrm{H} & -4.094709 & -5.510760 & -0.529479 \\ \mathrm{H} & -5.606751 & -5.348426 & -1.434496 \\ \mathrm{H} & -4.062645 & -5.075007 & -2.256634 \\ \mathrm{C} & -6.014609 & 2.904848 & 0.853053 \\ \mathrm{H} & -6.133122 & 2.092743 & 1.581970 \\ \mathrm{H} & -6.785114 & 3.650275 & 1.051209 \\ \mathrm{H} & -6.186597 & 2.499633 & -0.152322 \\ \mathrm{C} & -4.604951 & 4.935876 & 1.312609 \\ \mathrm{H} & -5.606746 & 5.348429 & 1.434499 \\ \mathrm{H} & -4.062639 & 5.075010 & 2.256632 \\ \mathrm{H} & -4.094708 & 5.510762 & 0.529477 \\ \mathrm{~F} & 4.191717 & -3.223824 & -2.272681 \\ \mathrm{~F} & 3.477851 & -3.608372 & 2.329584 \\ \mathrm{~F} & 3.477866 & 3.608364 & -2.329579 \\ \mathrm{~F} & 4.191717 & 3.223817 & 2.272689\end{array}$


Table S7b. Fully-optimized geometry of the internal charge-transfer triplet excited state of complex 4F (centered on the ancillary ligand) in acetonitrile (see Experimental Section for further details). The reported $x y z$ Cartesian coordinates are in Ångström.

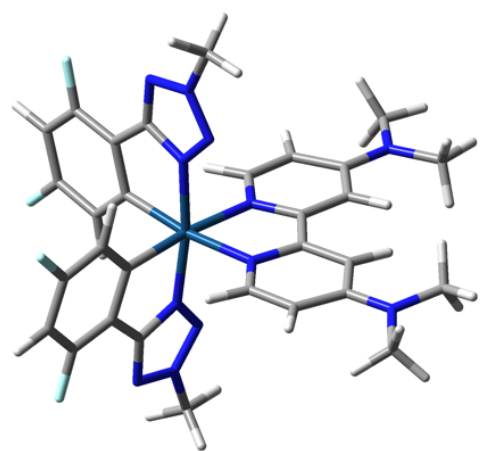

\begin{tabular}{|c|c|c|}
\hline-3.734902 & 582127 & \\
\hline-3.004594 & -3.187113 & -1.147557 \\
\hline-2.079880 & -2.149287 & -1.082424 \\
\hline-1.851993 & -1.448307 & 0.132637 \\
\hline-2.590630 & -1.845733 & 1.249225 \\
\hline-3.501034 & -2.888951 & 1.137706 \\
\hline-4.453897 & -4.392005 & -0.096295 \\
\hline-2.477372 & -1.365683 & 2.219692 \\
\hline-1.271537 & -1.690499 & -2.183748 \\
\hline-0.414446 & -0.669406 & -1.895343 \\
\hline-1.152171 & -2.028386 & -3.459826 \\
\hline 0.247096 & -0.358411 & -2.979040 \\
\hline-0.217157 & -1.183893 & -3.886407 \\
\hline 0.262431 & -1.179296 & -5.256970 \\
\hline 0.712392 & -2.148029 & -5.480699 \\
\hline-0.578531 & -0.993320 & -5.926941 \\
\hline 1.003012 & -0.385436 & -5.344783 \\
\hline-0.419664 & 0.013200 & 0.016070 \\
\hline-1.927480 & 1.367000 & -0.270885 \\
\hline-2.371416 & 2.016355 & 0.913376 \\
\hline-2.535938 & 1.738037 & -1.471778 \\
\hline-3.373695 & 2.980907 & 0.868288 \\
\hline-3.531608 & 2.706674 & -1.468910 \\
\hline-2.252786 & 1.292430 & -2.423990 \\
\hline-3.975814 & 3.348915 & -0.319600 \\
\hline-4.755735 & 4.101427 & -0.352784 \\
\hline-1.686552 & 1.586381 & 2.105936 \\
\hline-0.710968 & 0.649871 & 1.917187 \\
\hline-1.780235 & 1.881693 & 3.394526 \\
\hline-0.188773 & 0.349550 & 3.078483 \\
\hline-0.846587 & 1.098416 & 3.929298 \\
\hline-0.568555 & 1.080477 & 5.354462 \\
\hline 0.215588 & 0.344912 & 5.528588 \\
\hline-1.478361 & 0.804335 & 5.889783 \\
\hline-0.237813 & 2.073002 & 5.665224 \\
\hline 1.260827 & -1.234413 & 0.432249 \\
\hline 2.487470 & -0.628308 & 0.264645 \\
\hline 1.224498 & -2.521211 & 0.807164 \\
\hline 2.464360 & 0.743480 & -0.108743 \\
\hline 3.671100 & -1.365266 & 0.471546 \\
\hline 2.339003 & -3.292392 & 1.035979 \\
\hline 0.22923 & -2.947705 & 0.930269 \\
\hline 3.604710 & 1.549932 & -0.295381 \\
\hline 1.17858 & 1.332550 & -0.269589 \\
\hline 4.6 & -0.8 & 0.3225 \\
\hline
\end{tabular}
3.625422
$-2.708033$
0.860934
2.212508
3.489447
4.583411
1.077224
2. 157547
0.064948
1.991612
4.758883
4.581435
5. 911172
5.907766
6.600145
6.256576
4.533632
3.577220
5.318498
4. 729881
6.055751
6.197498
6.837755
6.193152
4.670435
5. 677318
4.159588
4.137909
$-4.324660$
2. 891494
1.118276
2. 617432
3. 450822
2. 995798
4.463864
$-3.436780$
3. 677009
3.094570
2.193695
3. 822522
2. 837119
5.124395
5.523907
5.547054
5.427327
$-2.817839$
$-1.967334$
$-3.549737$
$-2.456589$
$-4.824806$
$-5.231177$
$-4.934095$
$-5.435931$
$-4.096612$
3. 046827
3. 568186
$-3.758951$
$-3.186443$
$-3.819219$
$-4.192560$
1.339728
$-0.641880$
$-0.118956$
$-0.622739$
$-0.819667$
$-0.755974$
$-1.162441$
1.066687
$-0.840928$
$-0.891334$
$-1.510221$
$-1.322030$
0.118364
$-0.954700$
$-0.625535$
$-0.319331$
$-1.990344$
0.887023
1.568445
1.093918
$-0.141635$
1.477042
1. 583464
2. 443181
0.736195
$-2.629510$
2. 001890
$-2.307560$
2. 220244 
Table S7c. Fully-optimized geometry of the oxidized form of complex 4F in acetonitrile (see Experimental Section for further details). The reported $x y z$ Cartesian coordinates are in Ångström.

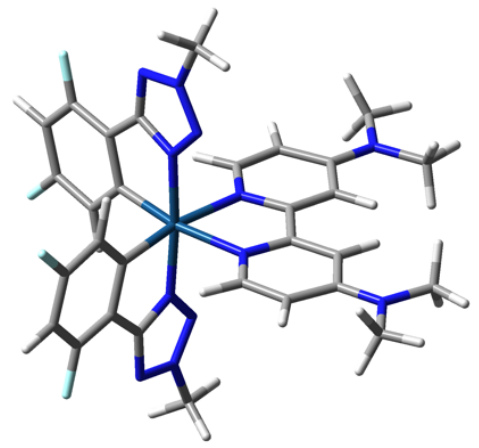

\begin{tabular}{|c|c|c|}
\hline 3.698810 & 3.626335 & 0.228980 \\
\hline 3.059246 & 3.249565 & -0.943562 \\
\hline 2.158346 & 2.197241 & -0.965568 \\
\hline 1.881124 & 1.459627 & 0.228062 \\
\hline 2.518927 & 1.846424 & 1.414517 \\
\hline 3.409158 & 2.908401 & 1.386755 \\
\hline 4.399587 & 4.454232 & 0.242709 \\
\hline 2.342365 & 1.339806 & 2.359799 \\
\hline 1.438220 & 1.738939 & -2.127290 \\
\hline 0.611473 & 0.680616 & -1.913535 \\
\hline 1.371217 & 2.111364 & -3.395187 \\
\hline 0.012934 & 0.378848 & -3.034465 \\
\hline 0.491396 & 1.247481 & -3.891076 \\
\hline 0.088640 & 1.260571 & -5.288923 \\
\hline-0.358443 & 2.229341 & -5.515908 \\
\hline 0.970587 & 1.096647 & -5.909638 \\
\hline-0.635537 & 0.459653 & -5.429648 \\
\hline 0.524284 & -0.000002 & 0.000002 \\
\hline 1.881124 & -1.459635 & -0.228029 \\
\hline 2.158330 & -2.197240 & 0.965611 \\
\hline 2.518956 & -1.846434 & -1.414470 \\
\hline 3.059236 & -3.249560 & 0.943627 \\
\hline 3.409188 & -2.908408 & -1.386685 \\
\hline 2.342415 & -1.339818 & -2.359757 \\
\hline 3.698821 & -3.626335 & -0.228901 \\
\hline 4.399601 & -4.454230 & -0.242613 \\
\hline 1.438181 & -1.738934 & 2.127317 \\
\hline 0.611438 & -0.680612 & 1.913542 \\
\hline 1.371165 & -2.111343 & 3.395218 \\
\hline 0.012962 & -0.378765 & 3.034485 \\
\hline 0.491224 & -1.247551 & 3.891053 \\
\hline 0.088288 & -1.260767 & 5.288846 \\
\hline-0.635894 & -0.459850 & 5.429554 \\
\hline 0.970163 & -1.096907 & 5.909678 \\
\hline-0.358836 & -2.229550 & 5.515698 \\
\hline-1.183583 & 1.277922 & 0.319510 \\
\hline-2.412510 & 0.713599 & 0.191465 \\
\hline-1.119378 & 2.579747 & 0.665795 \\
\hline-2.412513 & -0.713578 & -0.191502 \\
\hline-3.569565 & 1.430864 & 0.407456 \\
\hline-2.223272 & 3.360841 & 0.89228 \\
\hline-0.123770 & 3.003918 & 0.76996 \\
\hline-3.569570 & -1.430836 & -0.40750 \\
\hline-1.183588 & -1.277910 & -0.319534 \\
\hline-4.52814 & 0.938056 & 0.30366 \\
\hline
\end{tabular}

\subsection{4}

4.397537

$-2.798925$

$-0.938021$

$-2.579736$

$-3.360821$

$-3.003913$

$-4.397518$

3. 522265

$-3.522227$

$-2.902948$

$-2.079651$

$-3.647969$

$-2.518119$

$-4.926632$

$-5.502513$

$-5.340802$

$-5.055299$

2.902996

2. 079702

3. 648023

2. 518164

4. 926668

5.340848

5.055332

5.502545

$-3.268449$

$-3.914094$

3. 914105

3. 268440
0.766046

1.170683

$-0.766100$

$-0.303729$

$-0.665820$

$-0.892322$

$-0.769976$

$-1.170723$

0.975965

$-0.976034$

$-0.846475$

$-1.560412$

$-1.051309$

0.167997

$-1.341248$

$-0.568246$

$-1.450688$

$-2.294107$

0.846392

1.560330

1.051213

$-0.168081$

1. 341180

1.450607

2.294045

0.568185

$-2.508300$

2. 061510

$-2.061437$

2. 508383 
Table S8a. Fully-optimized ground-state geometry of complex 5F in acetonitrile (see Experimental Section for further details). The reported $x y z$ Cartesian coordinates are in Ångström.

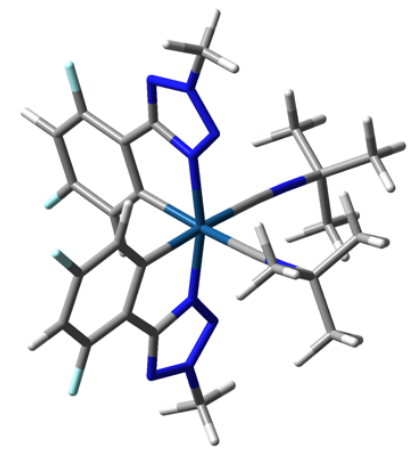

\begin{tabular}{|c|c|c|c|}
\hline $\mathrm{C}$ & -3.414803 & -3.175266 & 0.665015 \\
\hline $\mathrm{C}$ & -2.538333 & -2.780324 & 1.657421 \\
\hline & -1.578036 & -1.796746 & 1.436183 \\
\hline & -1.465377 & -1.169351 & 0.170148 \\
\hline & -2.352153 & -1.556221 & -0.831132 \\
\hline & -3.296076 & -2.541454 & -0.56469 \\
\hline & -4.162260 & -3.940934 & 0.840360 \\
\hline & -2.339655 & -1.111548 & -1.824825 \\
\hline & -0.646289 & -1.314274 & 2.42 \\
\hline & 0.184546 & -0.311866 & 2.01 \\
\hline & -0.384421 & -1.625841 & 3.685 \\
\hline & 0.973748 & 0.008888 & \\
\hline & 0.606466 & -0.789210 & 3.9 \\
\hline & 1.234436 & -0.755914 & 5.25 \\
\hline & 0.486775 & -0.4807 & \\
\hline & 1.63989 & -1.744166 & 5.5 \\
\hline & 2.03103 & -0.013934 & 5.25 \\
\hline & 0.04913 & 0.265 & 0.0 \\
\hline & 1.33740 & -1.25 & -0.5 \\
\hline & 1.39310 & -1.411311 & -1.96 \\
\hline & 2.11192 & $-2.1069^{\circ}$ & 0.2 \\
\hline & 2.19 & -2 . & -2.5 \\
\hline & 2.897862 & -3.074155 & -0.39 \\
\hline & 2.13147 & -2 . & 1.3 \\
\hline & 2.96 & -3 & -1.7 \\
\hline & 3.585103 & -4.012 & -2.22 \\
\hline & 0.57 & -0.482 & -2.7 \\
\hline & -0.11543 & 0.42 & -1.9 \\
\hline & 0.307625 & -0.292947 & -3.9 \\
\hline & -0.82010 & 1.183432 & -2.7 \\
\hline & -0 & 0.7 & -3.9 \\
\hline & -1.11934 & 1.3073 & -5.15448 \\
\hline & -1.79 & 2.10 & -4.8 \\
\hline & -1.66256 & 0.53 & -5.69 \\
\hline & -0.31665 & 1.7056 & -5.77713 \\
\hline & 3.63373 & -3.88 & 0.37 \\
\hline & 2.22025 & -2.51657 & -3.868716 \\
\hline & -2.614828 & -3.351566 & 2.858350 \\
\hline & -4.136060 & -2 & -1.5 \\
\hline & -3.445160 & 3.260348 & 0.345369 \\
\hline & 3.743 & 3. & 0.636448 \\
\hline & -4.481413 & & -0.466780 \\
\hline & -4.094376 & 2.256761 & -1.465074 \\
\hline & -4.75 & & 0.03516 \\
\hline & -5.38 & & -0.57 \\
\hline & -3.026699 & 4.542490 & -0.363 \\
\hline
\end{tabular}

$\begin{array}{rrrr}\mathrm{H} & -3.899491 & 5.197342 & -0.457616 \\ \mathrm{H} & -2.254330 & 5.070627 & 0.204936 \\ \mathrm{H} & -2.644261 & 4.324967 & -1.366933 \\ \mathrm{C} & -3.939258 & 3.540635 & 1.758366 \\ \mathrm{H} & -4.205607 & 2.610711 & 2.271414 \\ \mathrm{H} & -3.176422 & 4.064336 & 2.343689 \\ \mathrm{H} & -4.830627 & 4.174349 & 1.702315 \\ \mathrm{C} & 4.014531 & 2.908318 & 2.133419 \\ \mathrm{H} & 4.897909 & 3.509086 & 2.374704 \\ \mathrm{H} & 3.165143 & 3.289090 & 2.710466 \\ \mathrm{H} & 4.207197 & 1.870768 & 2.426924 \\ \mathrm{C} & 3.431139 & 4.441476 & 0.223783 \\ \mathrm{H} & 4.306654 & 5.066974 & 0.427789 \\ \mathrm{H} & 3.203477 & 4.500054 & -0.845609 \\ \mathrm{H} & 2.581686 & 4.835879 & 0.791106 \\ \mathrm{C} & 4.898613 & 2.436231 & -0.175567 \\ \mathrm{H} & 5.094152 & 1.395281 & 0.102649 \\ \mathrm{H} & 4.684657 & 2.483938 & -1.248306 \\ \mathrm{H} & 5.799041 & 3.026120 & 0.026217 \\ \mathrm{C} & 1.626157 & 1.534747 & 0.195610 \\ \mathrm{C} & -1.372017 & 1.670062 & 0.398113 \\ \mathrm{~N} & 2.567651 & 2.201348 & 0.360380 \\ \mathrm{~N} & -2.272234 & 2.409095 & 0.438173\end{array}$


Table S8b. Fully-optimized geometry of the ${ }^{3} \mathrm{LC}$ excited state of complex $5 \mathrm{~F}$ in acetonitrile (see Experimental Section for further details). The reported $x y z$ Cartesian coordinates are in Ångström.

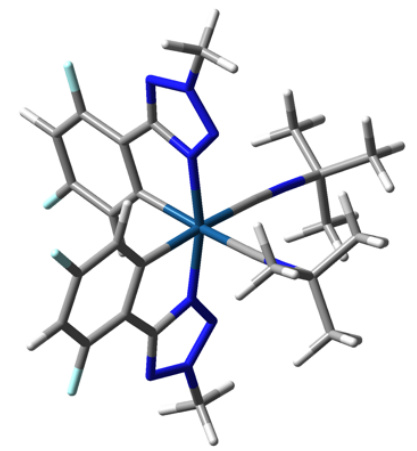

\begin{tabular}{|c|c|c|c|}
\hline $\mathrm{C}$ & 3.244220 & 3.334793 & 0.828479 \\
\hline $\mathrm{C}$ & 2.539981 & 2.688973 & 1.826575 \\
\hline$C$ & 1.638814 & 1.665928 & 1.542220 \\
\hline C & 1.411366 & 1.256185 & 0.204533 \\
\hline & 2.123938 & 1.897481 & -0.804731 \\
\hline & 3.013709 & 2.913101 & -0.47413 \\
\hline & 3.945808 & 4.130429 & 1.05313 \\
\hline & 2.013840 & 1.630823 & -1.85 \\
\hline & 0.875634 & 0.940808 & 2.52 \\
\hline & 0.066248 & -0.047001 & 2.04 \\
\hline & 0.759512 & 1.009746 & 3.8 \\
\hline & -0.565427 & -0.598295 & \\
\hline & -0.127019 & 0.05140 & 4.0 \\
\hline & -0.574673 & -0.257837 & 5.44 \\
\hline & 0.281826 & -0.5831 & \\
\hline & -1.016475 & 0.6378 & 5.8 \\
\hline & -1.314419 & -1.0539 & 5.37 \\
\hline Ir & -0.000357 & -0.269 & 0.0 \\
\hline & -1.44872 & 1.212 & -0.1 \\
\hline & -1.663533 & 1.623807 & -1.57 \\
\hline & -2.18475 & 1.850 & 0.8 \\
\hline & -2.615887 & 2.686 & -1.87 \\
\hline & -3.105994 & 2.8727 & 0.44 \\
\hline & -2.100 & 1.60 & 1.8 \\
\hline & -3.320723 & 3.297 & -0.9 \\
\hline & -4.031254 & 4.0872 & -1.12 \\
\hline & -0.905 & 0.928 & -2.5 \\
\hline & -0.059094 & -0.059 & -1.9 \\
\hline & -0.761533 & 0.973 & -3.8 \\
\hline & 0.585 & -0.599 & -2.9 \\
\hline & 0.148 & 0 & -4.0 \\
\hline & 0.636961 & -0.298802 & -5.38 \\
\hline & 1.371654 & -1.0959 & -5.2 \\
\hline & 1.09501 & 0.588 & -5.8 \\
\hline & -0.201560 & -0.6295 & -6.001274 \\
\hline & -3.816273 & 3.472188 & 1.3 \\
\hline & -2.751484 & 3.01750 & -3.16 \\
\hline & 2.729394 & 3.051478 & 3.09 \\
\hline $\mathrm{F}$ & 3.686 & 3.51 & -1.4 \\
\hline $\mathrm{C}$ & 3.569839 & -3.192252 & -0.428175 \\
\hline & -3.500226 & -3.295 & 0.39480 \\
\hline & 4.568503 & -2.396636 & -1.2 \\
\hline & 4.126854 & -2.096588 & -2.217188 \\
\hline & 4.89 & -1.500 & -0.72466 \\
\hline & 5.4 & -3.024759 & -1.4 \\
\hline & 3.07 & -4.416733 & -1.186 \\
\hline
\end{tabular}

$\begin{array}{rrrr}\mathrm{H} & 3.920793 & -5.076401 & -1.391688 \\ \mathrm{H} & 2.335106 & -4.970963 & -0.594697 \\ \mathrm{H} & 2.616604 & -4.126391 & -2.139403 \\ \mathrm{C} & 4.155942 & -3.575224 & 0.925184 \\ \mathrm{H} & 4.482682 & -2.686567 & 1.475106 \\ \mathrm{H} & 3.422883 & -4.119031 & 1.529981 \\ \mathrm{H} & 5.024036 & -4.223078 & 0.764154 \\ \mathrm{C} & -4.007217 & -3.718869 & -0.978177 \\ \mathrm{H} & -4.859278 & -4.394439 & -0.847935 \\ \mathrm{H} & -4.336832 & -2.850951 & -1.558622 \\ \mathrm{H} & -3.227374 & -4.246241 & -1.537662 \\ \mathrm{C} & -4.562870 & -2.519289 & 1.164728 \\ \mathrm{H} & -5.427837 & -3.170180 & 1.331093 \\ \mathrm{H} & -4.178664 & -2.193880 & 2.137471 \\ \mathrm{H} & -4.890916 & -1.640280 & 0.599850 \\ \mathrm{C} & -2.998676 & -4.490408 & 1.196243 \\ \mathrm{H} & -2.213569 & -5.025224 & 0.651503 \\ \mathrm{H} & -2.604444 & -4.171071 & 2.166842 \\ \mathrm{H} & -3.833304 & -5.178362 & 1.368383 \\ \mathrm{C} & -1.481135 & -1.663076 & 0.064995 \\ \mathrm{C} & 1.514107 & -1.618874 & -0.045060 \\ \mathrm{~N} & -2.373573 & -2.401568 & 0.192683 \\ \mathrm{~N} & 2.426198 & -2.330023 & -0.188967\end{array}$


Table S9a. Fully-optimized ground-state geometry of complex $1 \mathrm{H}$ in acetonitrile (see Experimental Section for further details). The reported $x y z$ Cartesian coordinates are in Ångström.

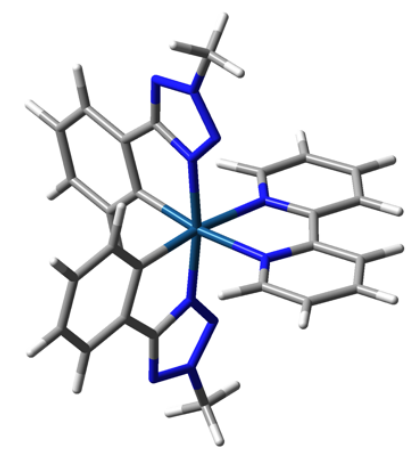

\begin{tabular}{lrrr}
$\mathrm{C}$ & 1.756762 & 3.464751 & -2.979656 \\
$\mathrm{C}$ & 2.547080 & 2.814678 & -2.042136 \\
$\mathrm{C}$ & 1.965366 & 1.854422 & -1.214120 \\
$\mathrm{C}$ & 0.590848 & 1.506779 & -1.282710 \\
$\mathrm{C}$ & -0.171927 & 2.184385 & -2.240812 \\
$\mathrm{C}$ & 0.401754 & 3.144308 & -3.074248 \\
$\mathrm{H}$ & 2.189729 & 4.215629 & -3.635175 \\
$\mathrm{H}$ & -1.234575 & 1.963998 & -2.347808 \\
$\mathrm{C}$ & 2.688331 & 1.102688 & -0.214899 \\
$\mathrm{~N}$ & 1.965756 & 0.178325 & 0.478675 \\
$\mathrm{~N}$ & 3.943109 & 1.101047 & 0.210167 \\
$\mathrm{~N}$ & 2.753751 & -0.409042 & 1.341763 \\
$\mathrm{~N}$ & 3.919714 & 0.164473 & 1.154207 \\
$\mathrm{C}$ & 5.097624 & -0.205212 & 1.918151 \\
$\mathrm{H}$ & 5.862902 & -0.579210 & 1.235862 \\
$\mathrm{H}$ & 5.467521 & 0.672976 & 2.449875 \\
$\mathrm{H}$ & 4.803489 & -0.981604 & 2.623228 \\
$\mathrm{I} r$ & 0.000000 & 0.037234 & -0.000001 \\
$\mathrm{C}$ & -0.590831 & 1.506784 & 1.282710 \\
$\mathrm{C}$ & -1.965345 & 1.854441 & 1.214121 \\
$\mathrm{C}$ & 0.171952 & 2.184379 & 2.240813 \\
$\mathrm{C}$ & -2.547049 & 2.814701 & 2.042141 \\
$\mathrm{C}$ & -0.401719 & 3.144305 & 3.074252 \\
$\mathrm{H}$ & 1.234597 & 1.963981 & 2.347809 \\
$\mathrm{C}$ & -1.756724 & 3.464762 & 2.979663 \\
$\mathrm{H}$ & -2.189683 & 4.215643 & 3.635184 \\
$\mathrm{C}$ & -2.688319 & 1.102716 & 0.214899 \\
$\mathrm{~N}$ & -1.965753 & 0.178348 & -0.478676 \\
$\mathrm{~N}$ & -3.943097 & 1.101090 & -0.210167 \\
$\mathrm{~N}$ & -2.753755 & -0.409009 & -1.341766 \\
$\mathrm{~N}$ & -3.919712 & 0.164518 & -1.154208 \\
$\mathrm{C}$ & -5.097626 & -0.205154 & -1.918154 \\
$\mathrm{H}$ & -4.803499 & -0.981548 & -2.623231 \\
$\mathrm{~N}$ & -0.389402 & -1.670261 & 1.273962 \\
$\mathrm{H}$ & -5.467512 & 0.673040 & -2.449878 \\
$\mathrm{H}$ & -5.862908 & -0.579143 & -1.235866 \\
$\mathrm{~N}$ & 0.389384 & -1.670266 & -1.273963 \\
$\mathrm{C}$ & 0.196050 & -2.885712 & -0.711105 \\
$\mathrm{C}$ & 0.741084 & -1.585320 & -2.563073 \\
$\mathrm{C}$ & -0.196083 & -2.885709 & 0.711105 \\
$\mathrm{C}$ & 0.357582 & -4.050079 & -1.456814 \\
$\mathrm{C}$ & 0.921694 & -2.706291 & -3.358622 \\
$\mathrm{H}$ & 0.875023 & -0.578749 & -2.954675 \\
$\mathrm{C}$ & -0.357628 & -4.050074 & 1.456815 \\
$\mathrm{H}$ & 0.192842 & -5.022964 & -1.006218 \\
\hline
\end{tabular}

$\begin{array}{rrrr}\mathrm{H} & 1.207321 & -2.590319 & -4.398680 \\ \mathrm{C} & -0.725033 & -3.959160 & 2.792165 \\ \mathrm{H} & -0.192900 & -5.022961 & 1.006220 \\ \mathrm{C} & -0.741102 & -1.585310 & 2.563072 \\ \mathrm{C} & -0.921726 & -2.706278 & 3.358621 \\ \mathrm{H} & -0.875030 & -0.578737 & 2.954674 \\ \mathrm{H} & -1.207352 & -2.590302 & 4.398679 \\ \mathrm{H} & 0.853058 & -4.860999 & -3.383282 \\ \mathrm{H} & -0.853116 & -4.860987 & 3.383283 \\ \mathrm{H} & -3.607822 & 3.041518 & 1.948787 \\ \mathrm{H} & 0.219206 & 3.651942 & 3.810014 \\ \mathrm{H} & -0.219165 & 3.651953 & -3.810008 \\ \mathrm{H} & 3.607855 & 3.041484 & -1.948781\end{array}$


Table S9b. Fully-optimized geometry of the ${ }^{3}$ MLCT excited state of complex $1 \mathrm{H}$ in acetonitrile (see Experimental Section for further details). The reported $x y z$ Cartesian coordinates are in Ångström.

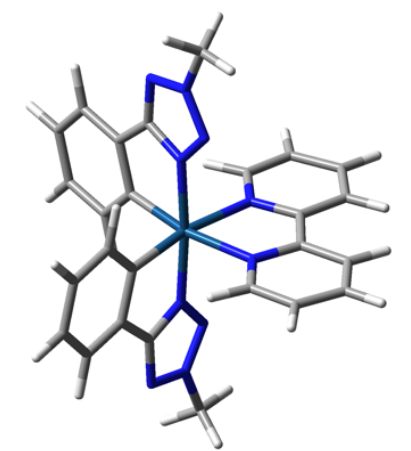

\begin{tabular}{|c|c|c|c|}
\hline $\mathrm{C}$ & 2.305478 & 3.250885 & -2.861700 \\
\hline $\mathrm{C}$ & 2.935798 & 2.635785 & -1.781041 \\
\hline$C$ & 2.207913 & 1.752843 & -0.997199 \\
\hline C & 0.831353 & 1.464857 & -1.248995 \\
\hline & 0.237512 & 2.095113 & -2.356312 \\
\hline & 0.963470 & 2.979862 & -3.144351 \\
\hline & 2.862650 & 3.940120 & -3.489971 \\
\hline & -0.802720 & 1.891122 & -2.604191 \\
\hline & 2.741249 & 1.036396 & 0.14056 \\
\hline & 1.871445 & 0.218075 & 0.78911 \\
\hline & 3.929154 & 0.977617 & 0.71 \\
\hline & 2.496370 & -0.3664 & 1.7 \\
\hline & 3.716767 & 0.111037 & 1.704 \\
\hline & 4.758050 & -0.279860 & 2.6400 \\
\hline & 5.585447 & -0.720 & \\
\hline & 5.097251 & 0.60466 & 3.1 \\
\hline & 4.327254 & -1.007504 & 3.32 \\
\hline Ir & 0.000001 & 0.128 & \\
\hline & -0.83136 & 1.464856 & 1.2 \\
\hline & -2.207924 & 1.752828 & $0.99^{\circ}$ \\
\hline & -0.237523 & 2.09512 & 2.3 \\
\hline & -2.93581 & 2.635 & $1.7 \xi$ \\
\hline & -0.963488 & 2.979869 & 3.14 \\
\hline & 0.802 & 1.8911 & 2.6 \\
\hline & -2.30549 & 3.25 & 2.8 \\
\hline & -2.862 & 3.94 & 3.48 \\
\hline & -2.74 & 1.03 & -0.1 \\
\hline & -1.871 & 0.21 & -0.7 \\
\hline & -3.929164 & 0.97 & -0.7 \\
\hline & -2.496 & -0.36 & $-1 \cdot 7$ \\
\hline & -3.716760 & 0.1110 & -1.7 \\
\hline & -4.758028 & -0.279841 & -2.64009 \\
\hline & -4.327221 & -1.0074 & -3.3 \\
\hline & -5.097224 & 0.60470 & -3.18 \\
\hline & -5.585432 & -0.720585 & -2.0821 \\
\hline & 0.624470 & -1.560196 & -1.1 \\
\hline & 0.327602 & -2.80056 & -0.62838 \\
\hline & 1.240274 & -1.495919 & -2.37710 \\
\hline & -0.327591 & -2.800567 & 0.62 \\
\hline C & 0.687206 & -3.970796 & -1.346061 \\
\hline & 1.603586 & -2.6050 & -3.1 \\
\hline & 1.438517 & -0.495346 & -2.7576 \\
\hline & -0.687196 & -3.970800 & 1.346057 \\
\hline & -0.624457 & -1.5602 & 1.17644 \\
\hline & 0.457480 & -4.945029 & -0.9 \\
\hline & 1.314006 & -3.881185 & -2.55 \\
\hline
\end{tabular}

$\begin{array}{rrrr}\mathrm{H} & 2.095378 & -2.487659 & -4.061087 \\ \mathrm{C} & -1.313998 & -3.881191 & 2.558950 \\ \mathrm{H} & -0.457472 & -4.945031 & 0.924314 \\ \mathrm{C} & -1.240262 & -1.495925 & 2.377112 \\ \mathrm{C} & -1.603576 & -2.605062 & 3.101435 \\ \mathrm{H} & -1.438502 & -0.495353 & 2.757644 \\ \mathrm{H} & -2.095368 & -2.487669 & 4.061087 \\ \mathrm{H} & 1.584150 & -4.783501 & -3.101059 \\ \mathrm{H} & -1.584143 & -4.783509 & 3.101052 \\ \mathrm{H} & -3.980311 & 2.838176 & 1.555203 \\ \mathrm{H} & -0.485683 & 3.464344 & 3.992021 \\ \mathrm{H} & 0.485662 & 3.464329 & -3.992030 \\ \mathrm{H} & 3.980290 & 2.838207 & -1.555202\end{array}$


Table S10a. Fully-optimized ground-state geometry of complex $3 \mathrm{H}$ in acetonitrile (see Experimental Section for further details). The reported $x y z$ Cartesian coordinates are in Ångström.

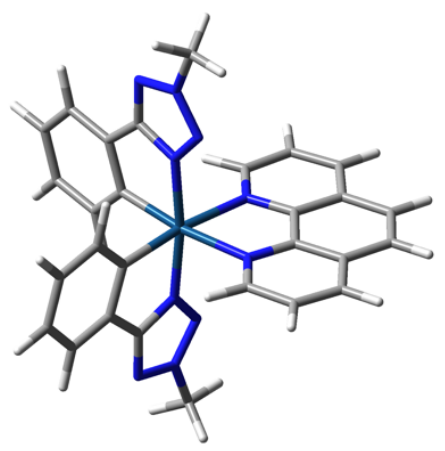

\begin{tabular}{|c|c|c|c|}
\hline $\mathrm{C}$ & -3.653418 & -1.906086 & 2.909414 \\
\hline$C$ & -3.008264 & -2.649423 & 1.930901 \\
\hline & -2.053488 & -2.027122 & 1.126351 \\
\hline & -1.706871 & -0.657298 & 1.260211 \\
\hline & -2.379707 & 0.057890 & 2.257261 \\
\hline & -3.334235 & -0.556685 & 3.067450 \\
\hline & -4.400046 & -2.371244 & 3.547510 \\
\hline & -2.160203 & 1.114512 & 2.413460 \\
\hline & -1.307239 & -2.698097 & \\
\hline & -0.389771 & -1.939790 & -0.57672 \\
\hline & -1.305432 & -3.930653 & -0.3982 \\
\hline & 0.193577 & -2.6832 & -1.4 \\
\hline & -0.375662 & -3.858525 & $-1 \cdot 3$ \\
\hline & -0.011566 & -4.995841 & -2.17 \\
\hline & 0.362361 & -5.796473 & -1.5 \\
\hline & -0.892466 & -5.335365 & -2.71 \\
\hline & 0.763333 & -4.667234 & -2.86 \\
\hline & -0.248706 & -0.000 & -0.0 \\
\hline & -1.70687 & 0.657 & $-1 \cdot 2$ \\
\hline & -2.053493 & 2.027116 & -1.12 \\
\hline & -2.379705 & -0.057 & $-2 \cdot 2$ \\
\hline & -3.00827 & 2.649 & -1.9 \\
\hline & -3.334234 & 0.556676 & -3.06 \\
\hline & -2.160 & -1.114 & -2.4 \\
\hline & -3.65342 & 1. & -2.9 \\
\hline & -4.400050 & 2.371 & -3.5 \\
\hline & -1.30724 & 2.698 & -0.0 \\
\hline & -0.38977 & 1.939 & 0.5 \\
\hline & -1.305 & 3.930 & 0.3 \\
\hline & 0.19 & 2.683 & 1.4 \\
\hline & -0.375676 & & 1.3 \\
\hline & -0.011589 & 4.9958 & 2.17 \\
\hline & 0.76 & 4.667243 & 2.8 \\
\hline & -0.89248 & 5.335 & 2.7 \\
\hline & 0.362303 & 5.796 & 1.53 \\
\hline & 1.454164 & -0.490656 & 1.2 \\
\hline & 2.65616 & -0.2580 & 0.6 \\
\hline & 1.428637 & -0.9638 & 2.4 \\
\hline a & 2.6 & & -0.6 \\
\hline $\mathrm{C}$ & 3.878433 & -0.505383 & 1.321787 \\
\hline & 2.597015 & -1.234 & 3.22 \\
\hline & 0.4 & -1.1 & 2.5 \\
\hline & 3.878432 & 0.505395 & -1.32178 \\
\hline & 1.454163 & 0.490 & $-1 \cdot 2$ \\
\hline & & -1.007242 & 2.6 \\
\hline & 2.518767 & -1.620356 & 4.2 \\
\hline
\end{tabular}


Table S10b. Fully-optimized geometry of the ${ }^{3} \mathrm{LC}$ excited state of complex $3 \mathrm{H}$ in acetonitrile (see Experimental Section for further details). The reported $x y z$ Cartesian coordinates are in Ångström.

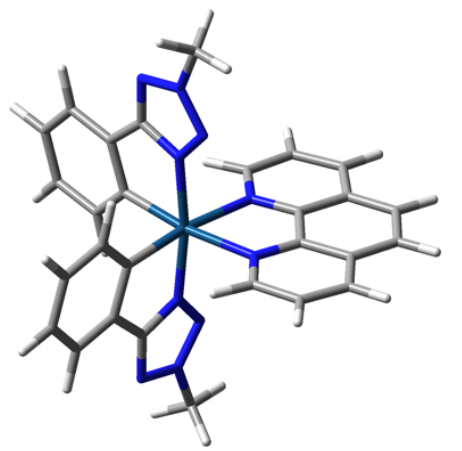

\begin{tabular}{|c|c|c|c|}
\hline $\mathrm{C}$ & 3.649746 & -1.816829 & -2.957149 \\
\hline$C$ & 3.008981 & -2.586650 & -1.996439 \\
\hline$C$ & 2.052058 & -1.988552 & -1.176405 \\
\hline $\mathrm{C}$ & 1.699404 & -0.617540 & -1.276654 \\
\hline & 2.367238 & 0.124811 & -2.256812 \\
\hline & 3.323841 & -0.465739 & -3.08239 \\
\hline & 4.397954 & -2.262809 & -3.60692 \\
\hline & 2.142421 & 1.183668 & -2.388215 \\
\hline & 1.308807 & -2.689278 & -0.15 \\
\hline & 0.384634 & -1.953415 & 0.52 \\
\hline & 1.315088 & -3.932380 & 0.3023 \\
\hline & -0.194551 & -2.7208 & 1.4 \\
\hline & 0.383201 & -3.88834 & 1.25 \\
\hline & 0.024840 & -5.047264 & 2.0484 \\
\hline & -0.343752 & -5.834342 & 1.3 \\
\hline & 0.907324 & -5.394 & 2.5 \\
\hline & -0.752634 & -4.739736 & 2.7 \\
\hline Ir & 0.235 & -0.000 & 0.0 \\
\hline & 1.699542 & 0 & 1.2 \\
\hline & 2.052805 & 1.9877 & 1.17 \\
\hline & 2.366 & -0.12 & 2.2 \\
\hline & 3.00 & 2 . & 1.9 \\
\hline & 3.323699 & 0.464 & 3.08 \\
\hline & 2.14 & -1.184 & 2.3 \\
\hline & 3.650 & 1. & 2.9 \\
\hline & 4.398 & 2.2610 & 3.60 \\
\hline & 1.30 & 2.68 & 0.1 \\
\hline & 0.38 & 1. & -0.52 \\
\hline & 1.31 & 3.9318 & -0.30 \\
\hline & -0.19 & 2.72 & -1.41 \\
\hline & 0.38 & 3. & -1.250 \\
\hline & 0.027418 & 5.0471 & -2.04872 \\
\hline & -0.750160 & 4.73 & -2.7 \\
\hline & 0.91012 & 5.393 & -2.588539 \\
\hline & -0.34081 & 5.8345 & -1.388840 \\
\hline & -1.471 & -0.426 & -1.2 \\
\hline & -2.65318 & -0.2280 & -0.695885 \\
\hline & -1.410104 & -0.837042 & -2.56584 \\
\hline a & -2.65 & 0.2 & \\
\hline C & -3.891960 & -0.439098 & -1.380164 \\
\hline & -2.561906 & -1.063242 & -3.31119 \\
\hline & -0.41 & -0 & -2.97503 \\
\hline & -3.891860 & 0.440513 & 1.380014 \\
\hline & -1.47 & & \\
\hline & -3.80 & -0.872090 & -2 . \\
\hline & -2.469940 & -1.389564 & -4.34 \\
\hline
\end{tabular}

$\begin{array}{lrrr}\mathrm{C} & -3.804584 & 0.873486 & 2.735970 \\ \mathrm{C} & -1.409913 & 0.837676 & 2.565761 \\ \mathrm{C} & -2.561665 & 1.064249 & 3.311077 \\ \mathrm{H} & -0.412054 & 0.971144 & 2.974981 \\ \mathrm{H} & -2.469624 & 1.390551 & 4.342232 \\ \mathrm{H} & -4.718842 & -1.044660 & -3.298498 \\ \mathrm{H} & -4.718606 & 1.046348 & 3.298320 \\ \mathrm{C} & -5.081383 & 0.222195 & 0.701716 \\ \mathrm{C} & -5.081433 & -0.220404 & -0.701899 \\ \mathrm{H} & -6.030064 & -0.378856 & -1.207680 \\ \mathrm{H} & -6.029978 & 0.380948 & 1.207469 \\ \mathrm{H} & 3.823952 & -0.140591 & 3.836493 \\ \mathrm{H} & 3.241747 & 3.642683 & 1.879137 \\ \mathrm{H} & 3.240366 & -3.643971 & -1.878902 \\ \mathrm{H} & 3.824446 & 0.139119 & -3.836057\end{array}$


Table S10c. Fully-optimized geometry of the ${ }^{3}$ MLCT excited state of complex $3 \mathrm{H}$ in acetonitrile (see Experimental Section for further details). The reported $x y z$ Cartesian coordinates are in Ångström.

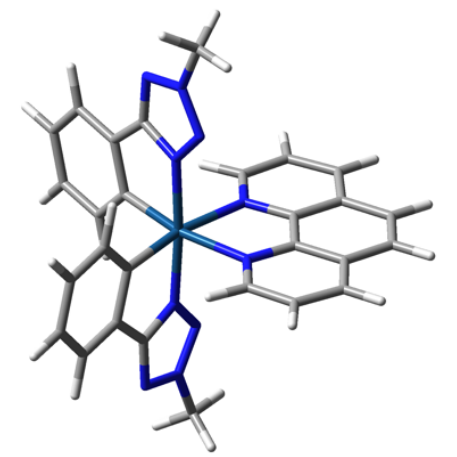

\begin{tabular}{|c|c|c|c|}
\hline $\mathrm{C}$ & -3.403432 & -2.386244 & 2.859293 \\
\hline $\mathrm{C}$ & -2.796727 & -2.991398 & 1.759537 \\
\hline & -1.934137 & -2.240250 & 0.975285 \\
\hline & -1.658794 & -0.864496 & 1.246095 \\
\hline & -2.279493 & -0.296434 & 2.37222 \\
\hline & -3.144337 & -1.045937 & 3.1603 \\
\hline & -4.076797 & -2.961949 & 3.48806 \\
\hline & -2.084461 & 0.742019 & \\
\hline & -1.227656 & -2.746426 & -0.1 \\
\hline & -0.427632 & -1.856847 & -0.8 \\
\hline & -1.163731 & -3.923725 & \\
\hline & 0.15 & -2.458692 & -1.82 \\
\hline & -0.312532 & -3.685209 & -1.77 \\
\hline & 0.07 & -4.706367 & -2.7 \\
\hline & 0.53676 & -5.537077 & -2.1 \\
\hline & -0.809540 & -5.047 & -3.26 \\
\hline & 0.790906 & -4.25 & -3.4 \\
\hline & -0.343864 & -0 & \\
\hline & -1.660961 & 0.860130 & -1.24 \\
\hline & -1.94 & 2.235149 & -0.9 \\
\hline & -2.27 & 0 . & -2 . \\
\hline & -2.804621 & 2.983828 & -1.76 \\
\hline & -3.146580 & 1.037233 & -3.1 \\
\hline & -2.082071 & -0.747783 & -2.63 \\
\hline & -3.409383 & 2.376876 & -2.8600 \\
\hline & -4.084149 & 2.950663 & -3.4 \\
\hline & -1.235313 & 2.743394 & 0 \\
\hline & -0.432972 & 1.856111 & 0.8250 \\
\hline & -1.17 & 3.92 & 0.7 \\
\hline & 0.14 & 2 . & 1.8 \\
\hline & -0.322953 & 3.684984 & 1.772 \\
\hline & 0.06 & 4.70 & 2.7 \\
\hline & 0.778724 & 4. & 3 . \\
\hline & -0.823613 & 5.046988 & 3.26417 \\
\hline & 0.521743 & 5.53 & 2.1 \\
\hline & 1.341600 & -0.653157 & 1.17 \\
\hline & 2.566603 & -0.334120 & 0.61082 \\
\hline & 1.33 & -1.29 & 2.36 \\
\hline & 2.565860 & & -0.61 \\
\hline & 3.790178 & -0.682065 & 1.25513 \\
\hline & 2.48 & -1.65 & 3.0 \\
\hline & 0.351695 & -1.520360 & 2.778940 \\
\hline & 3.788662 & 0.690877 & -1.254750 \\
\hline & 1.3401 & 0.6569 & -1.1 \\
\hline & 3.736228 & $-1 \cdot 34$ & 2.471 \\
\hline & 2.403123 & -2.16 & $3.98^{\circ}$ \\
\hline
\end{tabular}
3.733268
1. 329746
1.354167
1.298938
$-2.471269$
$-2.362284$
$1.660825-3.034876$
2.479454
1.522376
0.348361
2.398360
4.659691
4.656132
2.175136
$-2.778168$
$-3.986897$
$-1.618308$
2. 977992
5.013772
5.014492
1. 629153
0.330751
$-0.319553$
5.952381
$-0.583343$
$-2.977532$
$-0.595345$
0.595563
5.951078
0.596397
1.081246
$-1.081142$
$-3.622250$
$-2.989873$
$-3.000686$
$-0.588065$
$-4.034484$
4.026414
0.577930
4.022618
1. 519554
$-1.520421$
$-4.023143$ 
Table S11a. Fully-optimized ground-state geometry of complex $\mathbf{5 H}$ in acetonitrile (see Experimental Section for further details). The reported $x y z$ Cartesian coordinates are in Ångström.

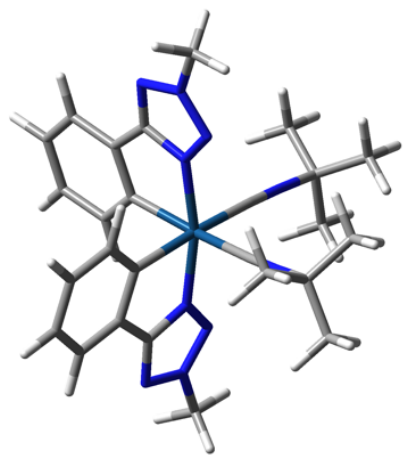

\begin{tabular}{|c|c|c|c|}
\hline C & -3.060664 & 3.395833 & 1.786876 \\
\hline $\mathrm{C}$ & -2.017779 & 3.716361 & 0.929171 \\
\hline & -1.185675 & 2.696215 & 0.466370 \\
\hline & -1.354702 & 1.342049 & 0.840193 \\
\hline & -2.419167 & 1.055034 & 1.696466 \\
\hline & -3.258434 & 2.067102 & 2.163521 \\
\hline & -3.721240 & 4.174282 & 2.15914 \\
\hline & -2.610297 & 0.028101 & 2.01064 \\
\hline & -0.082960 & 2.906939 & -0.442519 \\
\hline & 0.608261 & 1.799892 & -0.8 \\
\hline & 0.444087 & 3.982790 & -1.00 \\
\hline & 1.572613 & 2.166690 & -1.6380 \\
\hline & 1.442916 & 3.468209 & -1.7 \\
\hline & 2.323 & 4.28509 & -2.5 \\
\hline & 1.734781 & 4.774490 & -3.31 \\
\hline & 2.801612 & 5.032631 & -1.9 \\
\hline & 3.068472 & 3.625638 & -2.97 \\
\hline & 0.041718 & 0.028932 & 0.00 \\
\hline & 1.227556 & 0.3510 & 1.6 \\
\hline & 0.99 & -0.557 & 2.7 \\
\hline & 2.191285 & 1.336871 & 1.91 \\
\hline & 1.673 & -0.48 & 3.9 \\
\hline & 2.877043 & 1.413608 & 3.12 \\
\hline & 2.423505 & 2.062907 & 1.13 \\
\hline & 2.621 & 0.506878 & 4.1 \\
\hline & 3.163 & 0.578827 & 5.0 \\
\hline & -0.007178 & -1.551992 & 2.450862 \\
\hline & -0.55 & -1.50 & 1.2 \\
\hline & -0.551 & -2.544867 & 3.1 \\
\hline & -1.453261 & -2.451458 & 1.102345 \\
\hline & -1.41995 & -3.0498 & 2.2 \\
\hline & -2.26 & -4.189942 & 2.577923 \\
\hline & -2.894770 & -4.380137 & 1.709922 \\
\hline & -2.87 & -3.9 & 3.4 \\
\hline & -1.632643 & -5.053995 & 2.788798 \\
\hline & -3.481942 & -1.107119 & -2.740718 \\
\hline & 3.654391 & -2.1089 & -1.970 \\
\hline & -4.614935 & -0.941043 & -1.733353 \\
\hline & -4.441964 & -1.568074 & -0.85111 \\
\hline & -4.70 & 0.10 & $-1 \cdot 4$ \\
\hline & -5.558330 & -1.244797 & -2.199728 \\
\hline & -3.319138 & -2.564640 & -3.154380 \\
\hline & -4.234269 & -2.896694 & -3.656260 \\
\hline & -2.479408 & -2.683720 & -3.846847 \\
\hline & -3.151 & -3.201092 & -2.27876 \\
\hline & -3.6 & -0 . & -3 . \\
\hline
\end{tabular}

$\begin{array}{rrrr}\mathrm{H} & -3.760780 & 0.844054 & -3.649229 \\ \mathrm{H} & -2.842201 & -0.306389 & -4.654032 \\ \mathrm{H} & -4.599803 & -0.494027 & -4.465192 \\ \mathrm{C} & 4.292572 & -1.014739 & -2.819207 \\ \mathrm{H} & 5.178234 & -1.422409 & -3.318224 \\ \mathrm{H} & 3.594908 & -0.656746 & -3.583783 \\ \mathrm{H} & 4.601985 & -0.168777 & -2.196200 \\ \mathrm{C} & 3.189247 & -3.276660 & -2.832212 \\ \mathrm{H} & 4.060250 & -3.725773 & -3.321345 \\ \mathrm{H} & 2.699958 & -4.042660 & -2.221653 \\ \mathrm{H} & 2.491582 & -2.938731 & -3.605553 \\ \mathrm{C} & 4.598079 & -2.575521 & -0.869026 \\ \mathrm{H} & 4.901767 & -1.736688 & -0.233979 \\ \mathrm{H} & 4.123491 & -3.340129 & -0.245252 \\ \mathrm{H} & 5.493791 & -3.007368 & -1.328121 \\ \mathrm{C} & 1.556879 & -1.021860 & -0.844313 \\ \mathrm{C} & -1.347430 & -0.405612 & -1.406102 \\ \mathrm{~N} & 2.484188 & -1.531820 & -1.334902 \\ \mathrm{~N} & -2.261811 & -0.702168 & -2.067096 \\ \mathrm{H} & -1.845463 & 4.744045 & 0.614212 \\ \mathrm{H} & -4.080223 & 1.814951 & 2.830827 \\ \mathrm{H} & 3.623002 & 2.192813 & 3.273378 \\ \mathrm{H} & 1.457166 & -1.211342 & 4.758528\end{array}$


Table S11b. Fully-optimized geometry of the ${ }^{3} \mathrm{LC}$ excited state of complex $\mathbf{5 H}$ in acetonitrile (see Experimental Section for further details). The reported $x y z$ Cartesian coordinates are in Ångström.

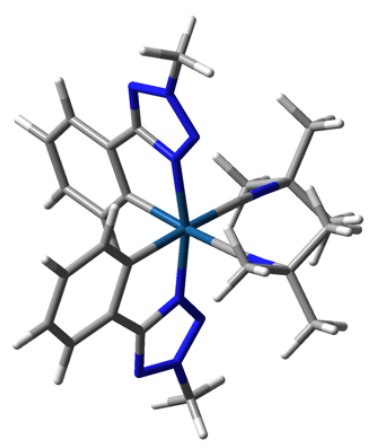

\begin{tabular}{|c|c|c|c|}
\hline C & -2.693025 & 1.954385 & 3.559105 \\
\hline C & -1.600312 & 2.606737 & 3.004731 \\
\hline C & -0.886500 & 1.980251 & 1.982190 \\
\hline $\mathrm{C}$ & -1.228062 & 0.699257 & \\
\hline C & -2.338409 & 0.077069 & \\
\hline C & -3.059234 & 0.695488 & \\
\hline $\mathrm{H}$ & -3.262411 & 2.424172 & \\
\hline $\mathrm{H}$ & -2.659625 & -0.904539 & \\
\hline $\mathrm{C}$ & 0.250946 & 2.569225 & 1.31 \\
\hline N & 0.805553 & 1.849369 & \\
\hline$N$ & 0.91 & 3.70 & \\
\hline $\mathrm{N}$ & 1.8212 & 2.515 & -0.1 \\
\hline $\mathbb{N}$ & 1.8552 & 3.611 & \\
\hline $\mathrm{C}$ & 2.84 & & \\
\hline $\mathrm{H}$ & 2.3 & 5.57 & \\
\hline $\mathrm{H}$ & 3.40418 & 4.8 & 1.2 \\
\hline $\mathrm{H}$ & 3.50 & 4.3 & -0 . \\
\hline Ir & 0.01551 & -0.0 & -0 . \\
\hline $\mathrm{C}$ & 1.22359 & $-0 . \varepsilon$ & \\
\hline $\mathrm{C}$ & $0.7 \varepsilon$ & -2.2 & \\
\hline $\mathrm{C}$ & 2.32 & -0.3 & \\
\hline $\mathrm{C}$ & 1.46 & -2.96 & 2. \\
\hline C & 3.0 & -1.0 & \\
\hline $\mathrm{H}$ & 2.69 & 0.66 & 1 . \\
\hline $\mathrm{C}$ & 2.5 & -2 & \\
\hline $\mathrm{H}$ & 3.0 & -2. & 4.2 \\
\hline $\mathrm{C}$ & -0.27 & -2.70 & 1. \\
\hline $\mathrm{N}$ & -0 & -1.86 & 0 . \\
\hline $\mathrm{N}$ & -0 . & -3. & 1. \\
\hline N & -1.79 & -2.45 & -0. \\
\hline $\mathrm{N}$ & -1.879 & -3.62 & \\
\hline C & -2.8 & -4.6 & -0 . \\
\hline $\mathrm{H}$ & -3.49 & -4.15 & -1 \\
\hline $\mathrm{H}$ & -3.487602 & -4.85 & \\
\hline $\mathrm{H}$ & $-2 \cdot 3$ & -5.49 & -0 . \\
\hline $\mathrm{C}$ & -3.62989 & 1.03 & -2.6 \\
\hline C & 3.69661 & -0.71797 & -2.71 \\
\hline $\mathrm{C}$ & -4.7 & 0.7 & -1.6 \\
\hline $\mathrm{H}$ & -4.63239 & -0.215638 & -1.20 \\
\hline $\mathrm{H}$ & -4.64480 & 1.52 & -0.80 \\
\hline $\mathrm{H}$ & -5.6 & 0.8 & -2.0 \\
\hline C & -3.699822 & 0.00817 & -3.7834 \\
\hline $\mathrm{H}$ & -4.65988 & 0.11025 & -4.30 \\
\hline $\mathrm{H}$ & -2.89 & 0.16 & -4.5 \\
\hline $\mathrm{H}$ & -3.625 & -1.008960 & -3.38 \\
\hline C & -3.68 & 2.458391 & -3.20 \\
\hline
\end{tabular}

$\begin{array}{rrrr}\mathrm{H} & -3.602038 & 3.190739 & -2.395761 \\ \mathrm{H} & -2.881141 & 2.633047 & -3.927494 \\ \mathrm{H} & -4.645387 & 2.607126 & -3.711373 \\ \mathrm{C} & 3.784882 & -2.063396 & -3.420169 \\ \mathrm{H} & 4.752229 & -2.133951 & -3.928687 \\ \mathrm{H} & 3.707263 & -2.888178 & -2.704130 \\ \mathrm{H} & 2.991029 & -2.165199 & -4.167389 \\ \mathrm{C} & 4.765497 & -0.581667 & -1.633443 \\ \mathrm{H} & 5.755476 & -0.618378 & -2.100676 \\ \mathrm{H} & 4.660215 & 0.373438 & -1.105837 \\ \mathrm{H} & 4.690655 & -1.396997 & -0.905528 \\ \mathrm{C} & 3.760475 & 0.438096 & -3.703647 \\ \mathrm{H} & 2.969753 & 0.352542 & -4.456220 \\ \mathrm{H} & 3.656469 & 1.398038 & -3.185727 \\ \mathrm{H} & 4.729946 & 0.418081 & -4.212910 \\ \mathrm{C} & 1.456942 & -0.485318 & -1.389918 \\ \mathrm{C} & -1.410520 & 0.619362 & -1.348450 \\ \mathrm{~N} & 2.413606 & -0.642136 & -2.038485 \\ \mathrm{~N} & -2.358274 & 0.859685 & -1.984554 \\ \mathrm{H} & -1.299466 & 3.593275 & 3.353349 \\ \mathrm{H} & -3.920381 & 0.187923 & 3.512698 \\ \mathrm{H} & 3.869254 & -0.651740 & 3.566103 \\ \mathrm{H} & 1.108480 & -3.956767 & 3.113988\end{array}$

